To APpear in ApJS

Preprint typeset using $\mathrm{LAT}_{\mathrm{E}} \mathrm{X}$ style emulateapj v. 08/22/09

\title{
SPITZER IRS SPECTRA AND ENVELOPE MODELS OF CLASS I PROTOSTARS IN TAURUS
}

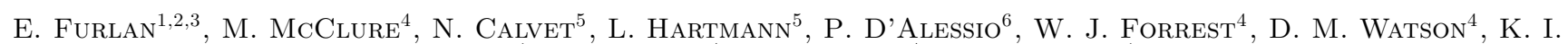 \\ UChida ${ }^{1}$, B. SArgent ${ }^{4}$, J. D. Green ${ }^{4}$, T. L. Herter ${ }^{1}$ \\ To appear in ApJS
}

\begin{abstract}
We present Spitzer Infrared Spectrograph spectra of 28 Class I protostars in the Taurus star-forming region. The 5 to $36 \mu \mathrm{m}$ spectra reveal excess emission from the inner regions of the envelope and accretion disk surrounding these predecessors of low-mass stars, as well as absorption features due to silicates and ices. Together with shorter- and longer-wavelength data from the literature, we construct spectral energy distributions and fit envelope models to 22 protostars of our sample, most of which are well-constrained due to the availability of the IRS spectra. We infer that the envelopes of the Class I objects in our sample cover a wide range in parameter space, particularly in density and centrifugal radius, implying different initial conditions for the collapse of protostellar cores.
\end{abstract}

Subject headings: circumstellar matter — stars: formation — stars: pre-main sequence — infrared: stars

\section{INTRODUCTION}

In a now widely accepted evolutionary sequence based on the shape of the spectral energy distribution (SED) in the infrared, there are four stages in the pre-mainsequence life of a low-mass star: Class 0 objects are deeply embedded protostars with large submillimeter to bolometric luminosity ratios, which are very faint at near-infrared wavelengths; Class I objects are surrounded by infalling envelopes, but are less embedded; Class II objects have cleared their envelopes and are surrounded by accretion disks; and Class III objects have dispersed almost all of their circumstellar material (Lada \& Wilking 1984; Lada 1987; André et al. 1993).

Therefore, Class I protostars are thought to be in one of the earliest evolutionary stages. After the gravitational collapse of a molecular cloud core starts, the infalling material forms an envelope around the central object. Since cloud cores are usually rotating slowly, the material falls preferentially onto a disk, which serves as a mass reservoir, most of which is eventually accreted by the protostar. Class I objects are thus surrounded by accretion disks, in addition to envelopes extending to several 1000 AU. The radius of the disk is roughly equal to the centrifugal radius $\left(R_{c}\right)$, which defines the centrifugal barrier encountered by matter falling in on the equatorial plane,

\footnotetext{
${ }^{1}$ Center for Radiophysics and Space Research, 208 Space Sciences Building, Cornell University, Ithaca, NY 14853; furlan@astro.ucla.edu, kuchida@astro.cornell.edu, tlh10@cornell.edu

${ }^{2}$ Current address: NASA Astrobiology Institute and Department of Physics and Astronomy, UCLA, 430 Portola Plaza, Los Angeles, CA 90095

3 NASA Postdoctoral Program Fellow

${ }^{4}$ Department of Physics and Astronomy, University of Rochester, Rochester, NY 14627; melisma@astro.pas.rochester.edu, forrest@pas.rochester.edu, dmw@pas.rochester.edu, bsargent@pas.rochester.edu, joel@pas.rochester.edu

${ }^{5}$ Department of Astronomy, The University of Michigan, 500 Church St., 830 Dennison Bldg., Ann Arbor, MI 48109; ncalvet@umich.edu, lhartm@umich.edu

${ }^{6}$ Centro de Radioastronomía y Astrofísica, UNAM, Apartado Postal 3-72 (Xangari), 58089 Morelia, Michoacán, México; p.dalessio@astrosmo.unam.mx
}

if the specific angular momentum is conserved; inside this radius the density distribution flattens, and the matter follows non-radial trajectories, falling onto a disk. Material tends to accumulate close to $R_{c}$, which is close to the protostar at the beginning of the collapse, but increases rapidly over time (Terebey, Shu, \& Cassen 1984; Adams \& Shu 1986).

Besides accreting matter, young stars generate powerful outflows which are launched along magnetic field lines; mass accretion onto the star and mass loss in the form of outflows seem to be correlated (e.g., Hartigan, Edwards, \& Ghandour 1995). In addition to collimated outflows, protostars might also emit a wideangle wind, which is expected to open up cavities in the envelope (Arce \& Goodman 2002). The presence of both collimated outflows and wide-angle stellar winds explains the fact that some objects with large cavities have well-collimated outflows. A partially evacuated cavity also forms as a natural by-product of the collapse of a sheet (Hartmann et al. 1994; Hartmann, Calvet, \& Boss 1996); since molecular cloud cores are rarely spherically symmetric, this scenario might be more realistic. In some cases, both stellar winds and the flattening of the envelope are responsible for cavities oriented along the rotational axis of a protostar.

The spectral index $n\left(\lambda F_{\lambda} \propto \lambda^{n}\right)$ from 2 to $25 \mu \mathrm{m}$ is used for the SED classification of protostars and premain-sequence stars (Lada 1987; Adams, Lada, \& Shu 1987; André \& Montmerle 1994); Class I objects have $n>0$, Class II objects have $-2<n<0$, and Class III objects have $-3<n<-2(n=-3$ is the spectral index of a photosphere in the Rayleigh-Jeans limit). Therefore, Class I objects are characterized by a strong infrared excess; the dust in the envelope absorbs radiation from the central protostar and disk and reemits it at infrared to $\mathrm{mm}$ wavelengths. The peak of the SED of this type of objects usually lies in the mid- to farinfrared, depending on the density of the envelope (and thus mass and infall rate), as well as the source luminosity (Kenyon. Calvet, \& Hartmann 1993a). Flat spectrum sources are likely objects surrounded by envelopes and disks and therefore protostars, but with wide cavi- 
ties in their envelopes and/or seen close to pole-on (e.g., DG Tau, T Tau; Furlan et al. 2006; Calvet et al. 1994). Some of the flat-spectrum sources could be transition objects (Class I/II), consisting of a T Tauri star and its disk surrounded by remnant envelope material (e.g., IRAS 04154+2823). Their SEDs typically show a double peak, one centered in the near-IR and one in the far-IR.

In addition to a rising SED in the infrared due to this excess emission, Class I protostars display various absorption features in their near- and mid-infrared spectra due to silicates and ices in the envelope. However, many parts of the infrared spectrum are not accessible from ground-based telescopes, which in addition often lack the sensitivity to observe faint objects. The Spitzer Space Telescope (Werner et al. 2004) opened a new window onto low-mass star formation; with unprecedented sensitivity, the precursors of low-mass stars can be studied in various evolutionary stages.

Here we present 28 spectra from 5 to $36 \mu \mathrm{m}$ of Class I objects in the Taurus star-forming region observed with the Infrared Spectrograph ${ }^{7}$ (IRS; Houck et al. 2004) on board of Spitzer. The 5-20 $\mu \mathrm{m}$ spectra of 5 of the 28 Class I objects were already introduced in Watson et al. (2004); these objects are IRAS $04016+2610,04108+2803 \mathrm{~B}, 04181+2654 \mathrm{~B}$, $04239+2436$, and DG Tau B. Our IRS observations are part of a larger Infrared Spectrograph guaranteed-time observing program; we presented the Class II and III objects from our sample of 150 young stellar objects in Taurus in an earlier paper (Furlan et al. 2006).

Since the Taurus-Auriga star-forming region is young (1-2 Myr) and relatively nearby (140 pc; Kenyon et al. 1994b; Bertout et al. 1999), many of its objects have been fairly well-studied at wavelengths covering almost the entire electromagnetic spectrum. Here we use SEDs constructed with data from the literature and our IRS spectra to constrain envelope models and to determine which parameters describe the circumstellar environment of the Class I objects in our sample.

This paper is structured as follows: in $\S 2$ we lay out our Spitzer IRS observations and data reduction; in $\S 3$ we present the IRS spectra and SEDs of the 28 Class I objects in our sample; in $\S 4$ we describe the envelope models used to fit 22 of our Class I objects and give detailed descriptions of the individual model fits; in $\S 5$ we discuss the different appearances of Class I objects and give a summary of our models; and in $\S 6$ we present our conclusions.

\section{OBSERVATIONS AND DATA REDUCTION}

Our Taurus targets were observed with the IRS on Spitzer during IRS observing campaigns 3 (2004 February 6 to 8), 4 (2004 February 27 to March 5), 12 (2004 August 30 to 31 ; only 3 targets), and 24 (2005 September 12 ; only IRAS $04166+2706)$. All targets were observed over the full IRS range from 5 to $40 \mu \mathrm{m}$ by using either the two low-resolution IRS modules (Short-Low [SL] and Long-Low [LL], 5.2-14 $\mu \mathrm{m}$ and $14-38 \mu \mathrm{m}$, respectively, $\lambda / \Delta \lambda \sim 90$ ) or the SL module and the two high-resolution modules (Short-High $[\mathrm{SH}]$ and Long-High

\footnotetext{
7 The IRS was a collaborative venture between Cornell University and Ball Aerospace Corporation, funded by NASA through the Jet Propulsion Laboratory and the Ames Research Center.
}

[LH], 10-19 $\mu \mathrm{m}$ and 19-37 $\mu \mathrm{m}$, respectively, $\lambda / \Delta \lambda \sim$ $600)$, depending on the object's expected mid-infrared flux. Thus, roughly half of our Class I objects were observed with SL and LL, and the other half with SL, SH, and LH. Our observations were carried out either in IRS staring mode, where the target is placed on two nod positions along the slit, at $1 / 3$ and $2 / 3$ of the slit length, or in mapping mode, with $2 \times 3$ step maps on the target (3 steps in the dispersion direction and 2 steps in the spatial direction). More than half of the objects (19 out of 28) were observed in mapping mode.

Data reduction was carried out using the SMART software tool (Higdon et al. 2004). Spectra were extracted after bad pixels, including so-called rogue pixels, were fixed by interpolation and after the sky in the lowresolution spectra was subtracted using the off-order observation (see Furlan et al. 2006 for more details). A variable-width column extraction was performed for lowresolution spectra, while high-resolution spectra were extracted using a full-slit extraction. The spectra were calibrated by dividing them by a calibrator spectrum ( $\alpha$ Lac (A1 V) for low-resolution and $\xi$ Dra (K2 III) for highresolution observations), and by multiplying the result by the calibrator's template spectrum (Cohen et al. 2003). Final spectra were obtained by averaging the two nod positions (for staring mode observations) or the two central map positions (for mapping mode observations). We also truncated all $\mathrm{SH}$ spectra below $13 \mu \mathrm{m}$ and rebinned all high-resolution spectra to a resolution of $\lambda / \Delta \lambda=200$ to achieve a more uniform representation of the spectra.

As mentioned in Furlan et al. (2006), we applied scalar corrections to each LH spectrum to account for the absence of sky subtraction in the high-resolution modules. Some minor adjustments $(\lesssim 10 \%)$ were also necessary for SL to match it to the flux level of SH. For IRAS $04154+2823,04158+2805,04325+2402$, and $04368+2557$, a multiplicative factor of 1.2 had to be applied to SL, while DG Tau B is the only Class I object in our sample to require an even larger scaling: a multiplication by 1.35 of SL2 $(5.2-7.5 \mu \mathrm{m})$ and by 1.21 of SL1 $(7.4-14 \mu \mathrm{m})$.

The flux mismatches between short- and longwavelength modules can be attributed to the extended nature of our targets; even though all objects in our sample appeared as point sources when observed with the IRS, we know that most of our targets are somewhat extended due to the presence of an envelope (e.g., Park \& Kenyon 2002). There are two effects caused by the extension of the envelope influencing our IRS observations.

First, the LL and LH slits, which are $10.6^{\prime \prime}$ and 11.1" wide, respectively, cover a larger area and thus more of the envelope emission than the SL and SH slits, which are $3.6^{\prime \prime}$ and $4.7^{\prime \prime}$ wide, respectively. However, the cold, outer envelope regions do not contribute much flux at the IRS wavelengths; at a distance of $140 \mathrm{pc}$, the SL slit encompasses all emission within $250 \mathrm{AU}$ from the central source, while LH includes emission out to 780 AU, sufficient to sample almost all of the material contributing to the IRS spectrum. Only a few objects probably suffer from slit losses beyond about $30 \mu \mathrm{m}$, evidenced by a change in slope, and thus decrease in flux level, around $30 \mu \mathrm{m}$; this effect is most notable in IRAS $04108+2803 \mathrm{~B}, 04158+2805,04181+2654 \mathrm{~A}$, 
$04239+2436$, and $04295+2251$.

Second, and more importantly, is the fact that protostellar envelopes are usually not spherically symmetric and that each of the IRS modules sample different regions of a protostellar system, since the slits of the various modules are not aligned parallel to each other and are also of different sizes (see, e.g., Fig. 1). For example, a protostar seen edge-on might show a dark central lane representing the disk and symmetric reflection nebulae representing inner envelope regions; if SL is aligned along the disk, LL will be oriented perpendicular to the dark lane and will sample more of the envelope emission.

The larger scalar correction factors applied to some of Class I objects in our sample (especially in the case of DG Tau B) are likely due to this second effect. In particular, the centering and orientation of the narrow SL slit could result in some of the envelope emission being missed by the SL observation (see Fig. 1). However, in most cases only a small fraction of the warm dust contributing at IRS wavelengths is not included in the IRS observations, and therefore the spectra we obtained are believed to be a good representation of the mid-infrared emission of the inner envelope regions.

Our absolute spectrophotometric accuracy is of the order of $5-10 \%$, depending on the module used and the pointing uncertainty. The relative spectral accuracy is module-dependent; spectral features above the noise level are likely real in SL, LL2 $(14-21 \mu \mathrm{m})$ and SH, while in LL1 $(20-36 \mu \mathrm{m})$ and especially in LH artifacts from unresolved calibration issues often do not allow reliable identifications of spectral features. Also the wavelength region where SL and LL meet $(13.5-14.5 \mu \mathrm{m})$ is affected by this higher uncertainty due to possible artifacts at the order edges.

Some of the Class I objects we observed with IRS are members of multiple systems (see Table 1). Except for GV Tau A and B, they either are at separations that place all members well within the IRS slits, or their separations are large enough to ensure that only one component is observed. Since the infrared emission from protostars is dominated by envelope emission, the multiplicity of the central source should have no impact on the midinfrared spectrum, except when the configuration of the system is such that it influences the emission from the envelope, e.g., by truncating it or causing a departure from spherical symmetry in the illuminating radiation field.

We encountered reduction problems in a few objects; they are listed below.

$04108+2803 B$. This object is part of a mispointed mapping-mode observation; by using the spectra from the central map position for SL and SH and scaled spectra from the third map position for LH, we created a continuous IRS spectrum. This stitching adds a higher uncertainty to the absolute flux level in our spectra, but the overall shape of the spectrum should be only minimally affected.

$G V \operatorname{Tau}(A, B)$. This binary is only separated by 1 .! 3 ; it was observed with SL, SH, and $\mathrm{LH}$ in mapping mode, and it was not resolved in any of the modules. Since this object consists of two point sources with likely some extended emission surrounding them, their SH and $\mathrm{LH}$ calibration presented some problems. The final spectrum was composed by using those nod and map positions for each module in which the flux was highest, and in ad- dition SH was scaled to match up with SL. Due to this procedure, the absolute flux level of this spectrum carries a higher uncertainty. In fact, we multiplied the entire spectrum by 0.85 to match fluxes at 11.7 and $17.9 \mu \mathrm{m}$ with our, yet unpublished, ground-based measurements at these wavelengths.

$H H$ 30. This object was too faint in SL2; the spectrum could only be extracted in SL1 and in LL.

LkHa 358. The peak-up arrays contained bright to saturated sources during the SL observations, which resulted in increased noise in the SL part of the spectrum. The LL observation included 3 sources, LkHa 358, HL Tau, and XZ Tau, but since the observation was centered on LkHa 358, the other two sources entered only partially in the slit. In addition, all three sources were separated sufficiently in the spatial direction to allow extraction of the individual spectrum of LkHa 358.

\section{THE SAMPLE \\ 3.1. IRS Spectra}

Our Taurus sample is based on objects analyzed by Kenyon \& Hartmann (1995) (from here on KH95); a large fraction of our Class I targets has identifiers from the IRAS Point Source Catalogue, since these objects were first identified as possible embedded protostars in the IRAS surveys and confirmed in follow-up observations (e.g., Beichman et al. 1986; Myers et al. 1987; Kenyon et al. 1990). Some objects that are part of our Class I sample are identified as Class II objects in KH95: these objects are IRAS 04154+2823, 04158+2805, 04278+2253, CoKu Tau/1, HL Tau, IC 2087 IR, and $\mathrm{LkHa} 358$. We base our identification of these sources as Class I objects on the existence of extended, nearinfrared nebulosity and possibly outflows around these objects, or the presence of a silicate absorption feature and a positive spectral index over the IRS range, or the detection of ice absorption features in the $5-8 \mu \mathrm{m}$ region as well as the $\mathrm{CO}_{2}$ ice feature at $15.2 \mu \mathrm{m}$. Therefore, this sample includes all Taurus objects we observed with the IRS that are thought to be at the protostellar evolutionary stage and therefore surrounded, to varying extents, by envelopes, even though in a few cases the SED slope does not lie in the typical range for Class I objects.

Figure 2 shows the IRS spectra of the 28 Class I objects in our sample. Most have SEDs that are rising over the IRS spectral range, and almost all of them show numerous absorption features in their mid-infrared spectra, typical of embedded protostars (e.g., Alexander et al. 2003; Gibb et al. 2004; Watson et al. 2004): wide bands due to amorphous silicates at 9.7 and $18 \mu \mathrm{m}$ (the latter one is much less prominent), and features at 6.0 $\mu \mathrm{m}$ due to $\mathrm{H}_{2} \mathrm{O}$ ice, at $15.2 \mu \mathrm{m}$ due to $\mathrm{CO}_{2}$ ice, and at $6.8 \mu \mathrm{m}$ due to organic compounds, possibly $\mathrm{CH}_{3} \mathrm{OH}$, $\mathrm{NH}_{4}^{+}$, or strongly polar $\mathrm{H}_{2} \mathrm{O}$ ice van Dishoeck 2004; Alexander et al. 2003). In addition, the silicate absorption feature at $9.7 \mu \mathrm{m}$ often displays a wider absorption shoulder at the longer-wavelength end due to the broad $\mathrm{H}_{2} \mathrm{O}$ ice libration band at $\sim 11-13 \mu \mathrm{m}$. The longwavelength wing of the $\mathrm{CO}_{2}$ ice feature also suggests that water ice is responsible, likely in the form of a mix with $\mathrm{CO}_{2}$ ice (Ehrenfreund et al. 1999).

Some protostars show an obvious self-absorbed silicate feature at $10 \mu \mathrm{m}$ (e.g., IRAS 04154+2823, 04181+2654 
B; see Fig. 2d), where the silicate emission from inner disk regions is absorbed in cooler envelope regions, causing a feature that is more or less flat, with some emission and some absorption characteristics (Mitchell \& Robinson 1981). The self-absorbed features probably indicate that the optical depth of the envelope along the line of sight is not as large as for the cases where a clear silicate absorption feature is seen.

IRAS 04248+2612, 04264+2433, and CoKu Tau/1 (Fig. 22) have a silicate emission feature, but are surrounded by nebulosities and have an overall rising SED in the infrared. Therefore, they are likely more evolved Class I objects seen through low-density envelopes, which have mostly dispersed and thus reveal the central star and its accretion disk. Based on the geometry of their near-infrared reflection nebulae, IRAS $04248+2612$ and $\mathrm{CoKu}$ Tau/1 are likely seen close to edge-on (Padgett et al. 1999).

\subsection{Spectral Energy Distributions}

Most protostars are found near or are embedded within dense molecular cloud cores (Myers et al. 1987; Onishi et al. 1998). Due to high extinction mostly due to their envelopes, protostars are usually faint or even undetectable in the optical. In the near-infrared and in the optical, the infalling envelope often appears as a reflection nebula, seen in light scattered by dust grains in the inner parts of envelope cavities (e.g., Tamura et al. 1991; Padgett et al. 1999; Park \& Kenyon 2002). In addition, Class I objects are often extended in the sub-mm and $\mathrm{mm}$ (Chandler et al. 1998; Motte \& André 2001); at these wavelengths, the thermal emission from cold dust in the envelope is detected.

To show the emission from protostellar systems at all wavelengths, in particular how the emitted power is distributed at different wavelengths, we generated spectral energy distributions with data from the literature. In Figure 3. we show the SEDs of the Class I objects in our sample, constructed with optical to mid-IR photometry mostly from Kenvon \& Hartmann (1995), the 2MASS $J, H$, and $K_{s}$ fluxes, the IRAS $12,25,60$, and $100 \mu \mathrm{m}$ fluxes, IRAC 3.6, 4.5, 5.8, and $8.0 \mu \mathrm{m}$ fluxes (Hartmann et al. 2005; Luhman et al. 2006), sub-mm and mm fluxes, where available, and the IRS spectrum. The IRAS fluxes are mostly from the IRAS Faint Source Catalog; for $\mathrm{CoKu}$ Tau/1, GV Tau, HL Tau, and L1551 IRS 5 we adopted the IRAS fluxes from Weaver \& Jones (1992), and for the 12 and $25 \mu \mathrm{m}$ fluxes of DG Tau B and HH 30 the measurements from coadded IRAS scans by Cohen \& Schwartz (1987). The fluxes at 350, 450, 800, 850, 1100, and 1300 $\mu \mathrm{m}$ are mostly from Moriarty-Schieven et al. (1994), Andrews \& Williams (2005), Young et al. (2003), and Motte \& André (2001), while the few measurements at $160 \mu \mathrm{m}$ were taken by Ladd et al. (1991) with the Kuiper Airborne Observatory (see Table 2 for a list of the longwavelength data used in the SED plots). The fluxes were not corrected for reddening, since the high extinction of these objects is very likely intrinsic.

The SED plots show that most of the power of a Class I object is emitted in the mid- to far-infrared; therefore, for many objects the IRS spectrum lies close to the peak of the emission. For most objects, the SED is either rising or flat over the IRS spectral range, with a deep silicate absorption feature at $10 \mu \mathrm{m}$ as the most notable spectral feature. Only a few objects have SEDs that seem to peak at $\sim 5 \mu \mathrm{m}$ and decrease over the IRS spectral range; they might be more evolved protostars and will be discussed in $\oint 3.3 .2$.

\subsection{Notes on Individual Objects Excluded in the Modeling \\ 3.3.1. Class I Objects}

GV Tau. This object, also known as Haro 6-10, consists of two components separated by 1 .'3. Like $\mathrm{T}$ Tau, the GV Tau system consists of a $\mathrm{T}$ Tauri star (component A) and an embedded infrared companion (component B). The binary is surrounded by a circumstellar disk and an almost edge-on circumbinary envelope, with outflows oriented perpendicular to the disk plane (Ménard et al. 1993). GV Tau B is likely responsible for the outflows measured from this source (Chandler et al. 1998; Hogerheijde et al. 1998). In the near-IR, the brightness ratio of the components varies considerably over the timescale of a few years (Leinert \& Haas 1989; Ménard et al. 1993; Koresko et al. 1997). It is likely that the B component, which is more deeply embedded, varies more than the A component in brightness and generates the deep 10 $\mu \mathrm{m}$ silicate absorption feature, as well as the various ice features, observed in its mid-IR spectrum (see Figure 2 c).

HH 30. Optical and near-IR HST images (Burrows et al. 1996; Cotera et al. 2001) reveal that this object is seen edge-on; the central star illuminates a flared circumstellar disk, which obscures the protostar and is seen in scattered light. In addition, a narrow jet is emitted perpendicular to the disk plane. Its appearance is reminiscent of that of DG Tau B, which is also seen edge-on and emits a jet. Wood et al. (1998) modeled the emission of $\mathrm{HH} 30$ and concluded that, in addition to a disk, only a tenuous envelope is required. The presence of a diffuse infalling envelope, as well as outflows, indicates that $\mathrm{HH} 30$ is a protostar, though likely more evolved.

The IRAS fluxes of $\mathrm{HH} 30$, determined by Cohen \& Schwartz (1987) from coadded IRAS scans, are highly uncertain, since they are contaminated by emission from two nearby sources, HL Tau and XZ Tau, which are much brighter. Our IRS spectrum shows that the IRAS fluxes were overestimated by 1.5 to 2 orders of magnitude (see Figure 3). HH 30 is among the faintest objects in our Taurus sample; its IRS spectrum suggests the presence of a deep silicate absorption feature, but its SED is only poorly determined.

\subsubsection{Class I/II Objects}

As indicated in $\S 3.2$, a few objects in our sample have SEDs that are not typical of protostars. IRAS 04278+2253 and IC 2087 IR have clearly decreasing SEDs from 5 to $40 \mu \mathrm{m}$ (see Fig. 2 $\mathrm{d}$ ) and would therefore be identified as Class II objects based on the slope of their infrared SED. Also IRAS 04154+2823 and LkHa 358 have SEDs that seem to peak around $5 \mu \mathrm{m}$ and do not display prominent $10 \mu \mathrm{m}$ silicate absorption features.

However, IC 2087 IR (also known as Elias 18) has ice absorption features in the $5-8 \mu \mathrm{m}$ spectral region, a 10 
$\mu \mathrm{m}$ silicate absorption feature, as well as a prominent $\mathrm{CO}_{2}$ ice absorption at $15.2 \mu \mathrm{m}$. It is likely surrounded by a circumstellar disk, probably seen edge-on given its high visual extinction $A_{V} \sim 18$ (Shuping et al. 2001). Bowey, Adamson. \& Yates (2003) measured the $10 \mu \mathrm{m}$ spectrum of IC 2087 IR; they noted a strong silicate absorption feature on a decreasing photosphere-like continuum. The absorption features we detect in the IRS spectrum of IC 2087 IR, and the fact that it is surrounded by a reflection nebulosity (IC 2087), suggest that it is actually a protostar, surrounded by an accretion disk and some envelope material. Further evidence for its earlier evolutionary stage are a molecular outflow (e.g., Heyer et al. 1987) and the presence of two Herbig-Haro knots, which are not within the outflow, but are oriented along the axis of symmetry of the reflection nebula (Gomez et al. 1997). It has been suggested that, since IC 2087 IR has a rather weak outflow, it could be in transition between the embedded protostellar and the $\mathrm{T}$ Tauri phase (Tegler et al. 1995).

In contrast to IC 2087 IR, IRAS $04278+2253$ shows no silicate absorption feature, only a weak $\mathrm{CO}_{2}$ ice feature, and likely gas absorption features in the $5-8 \mu \mathrm{m}$ wavelength range, most prominently an $\mathrm{H}_{2} \mathrm{O}$ band centered at $5.7 \mu \mathrm{m}$. This feature, similar to what is observed in some FU Ori objects (Green et al. 2006), is thought to arise in the upper layers of hot inner disk regions that are heated by accretion. IRAS $04278+2253$ is a $6{ }^{\prime \prime} 8$ binary, whose primary component is of G8 spectral type and has a relatively high mass accretion rate $\left(6.6 \times 10^{-6}\right.$ $\mathrm{M}_{\odot} \mathrm{yr}^{-1}$; White \& Hillenbrand 2004). The secondary is of K7/M0 spectral type (White \& Hillenbrand 2004) and thus clearly fainter than the primary. Given this object's relatively large mass accretion rate, it is likely still a protostar, but since no extended emission has been detected around it, there is likely only little envelope material left.

LkHa 358 forms a small cluster of stars together with HL Tau and XZ Tau (a flat-spectrum source, see Furlan et al. 2006): all three objects are found within a radius of less than $1^{\prime}$. All of them are likely embedded to some extent; while HL Tau has an obvious absorption feature and an SED typical of a Class I object, LkHa 358 only has a weak absorption feature at $10 \mu \mathrm{m}$, and XZ Tau has silicate emission features at 10 and $20 \mu \mathrm{m}$ and an overall flat SED in the mid-IR. Since HL Tau is the brightest source of the group, it is likely the dominating contribution to the IRAS source $04287+1807$; therefore, the IRAS fluxes for LkHa 358 are not known. The IRS spectrum shows that LkHa 358 has a silicate feature that could be partly self-absorbed, as well as some weak ice absorption features. The late spectral type of LkHa 358 (M5.5 according to Kenvon et al. 1998 and Luhman 2000) suggests that this object will end up as a very lowmass star, with a mass close to that of a brown dwarf.

IRAS $04154+2823$ is very faint and could be a more evolved protostar; in fact, its $10 \mu \mathrm{m}$ silicate feature seems to be self-absorbed, with a broad absorption centered at $9.7 \mu \mathrm{m}$ and emission between 10 and $12 \mu \mathrm{m}$, which is an indication of a tenuous envelope. Its SED can be fitted reasonably well with a low-density envelope model (see Figure 8).

\subsection{Model Description}

In this paper, we generated envelope models for 22 of the 28 Class I objects we observed in the Taurus starforming region using the IRS on Spitzer to characterize some of the envelope parameters that result in the observed SED shapes. These models include the contributions of the central protostar, the disk, and the envelope to compute the resulting SED of a protostellar system. We used a modeling code based on two types of models that differ in the initial configuration: so-called TSC models (after Terebey, Shu, \& Cassen 1984), which assume an initially spherically symmetric molecular cloud core, and sheet-collapse models (Hartmann et al. 1994; Hartmann, Calvet, \& Boss 1996), which simulate the collapse of elongated cloud cores. Both models result in flattened density distributions, in which the emergent SED is strongly dependent on the inclination angle of the system. Below, we will briefly introduce these two types of models.

\subsubsection{Envelope Component}

In the TSC models, the radiative equilibrium temperature is determined first by using the angle-averaged density distribution for the infall region $\left(\rho \propto r^{-3 / 2}\right.$ for $r \gg R_{c}, \rho \propto r^{-1 / 2}$ for $r \ll R_{c} ; R_{c}$, the centrifugal radius, is where material falling in on the equatorial plane lands and thus represents the outer disk radius). In this region, departures from spherical symmetry become apparent only at small distances, when material falls onto a disk; at large distances, the TSC model can be described by spherical free-fall. After the temperature distribution has been calculated, the flattened, axially symmetric density distribution from Terebev. Shu, \& Cassen (1984) is used to compute the flux emitted by the system (Kenyon, Calvet, \& Hartmann 1993a, from here on KCH93). The models also include isotropic scattering. The source luminosity is not set according to the mass infall rate, which means that the disk accretion rate can be different from the mass infall rate of the envelope.

Outflow cavities along the poles can also be included in the models by specifying a cavity semi-opening angle (Kenyon et al. 1993b; Calvet et al. 1994). Inside the bipolar cavities, material has been evacuated; outside, material will fall onto a disk with an inner radius $R_{\text {min }}=R_{c} \sin ^{2} \theta$, where $\theta$ is the half opening angle of the cavity, i.e. the angle between the polar axis and the streamline of an infalling particle at the edge of cavity at large distances, and $R_{c}$ is the centrifugal radius (Calvet et al. 1994). Thus, the shape of the cavities is determined by the streamlines of the infalling material.

The inclusion of a cavity in the density distribution of the envelope causes departures from spherical symmetry in the temperature distribution. Scattered light from these cavities adds flux at near-infrared wavelengths, but the cavities also remove hot, inner dust. Therefore, for larger cavities, the net result is a decrease in near- and mid-infrared flux when the inclination angle is larger than the opening angle of the cavity. However, when the inclination of the system allows the observer to see inside the cavity and thus the star and inner disk directly, the near-infrared emission increases (Calvet et al. 1994).

Sheet-collapse models start from the collapse of sheets that are initially in hydrostatic equilibrium 
(Hartmann et al. 1994; Hartmann, Calvet, \& Boss 1996). This modification to the spherical infall scenario produces partially evacuated cavities, which, when aligned along the line of sight, reveal the inner parts of the envelope and disk, thus increasing the object's optical and near-infrared emission. Since the polar regions of the sheet collapse before the equatorial regions, polar cavities, which are regions where low-density material is falling in, will result (Hartmann et al. 1994). With sheet collapse, the opening angle of the cavity is connected to the infall and not to the outflows; eventually, outflows, but also wide-angle stellar winds (Arce \& Goodman 2002), might easily remove the low-density material and fully clear out the cavities, so in the end both effects, sheet collapse and mass loss from a protostar, could be responsible for the formation of cavities.

\subsubsection{Disk Component}

The disk is modeled as an optically thick, steady accretion disk irradiated as a flat disk by the central star $\left(T(r) \propto r^{-3 / 4} ; T^{4}=T_{\text {accretion }}^{4}+T_{\text {irradiation }}^{4}\right)$, oriented perpendicular to the rotation axis of the envelope. As the outer disk regions, which emit mostly in the sub-mm and $\mathrm{mm}$, are optically thin, and only the inner disk regions are optically thick, the outer disk radius was set at $10 \mathrm{AU}$ to represent the optically thick part of the disk. However, the disk is expected to extend out to $R_{c}$; emission from the protostellar envelope will be obscured if it lies behind the disk along the line of sight. Therefore, to take this obscuration effect into account, a second set of models was calculated with the disk radius set equal to $R_{c}$. The latter set was used for the envelope and stellar emission, while the set with the smaller disk radius was used for the disk emission. In this way the disk component in our models represents only the optically thick disk regions; the optically thin outer regions would likely add small amounts of additional flux at sub-mm and mm wavelengths.

This simple disk model also does not include an inner disk wall at the dust truncation radius, which would add flux at near-IR wavelengths, in particular for systems with higher inclination angles $i$, where the disk emission is reduced due to its $\cos (i)$ dependence. In addition, since the disk model does not include an optically thin disk atmosphere that generates a $10 \mu \mathrm{m}$ silicate emission feature (see, e.g., D'Alessio et al. 1999), the shape of the silicate feature is only determined by the envelope. The material in the envelope will extinguish the disk and thus generate a $10 \mu \mathrm{m}$ absorption feature in the continuum emission arising from the disk, while the silicates in the envelope will produce an emission feature when the density is low and an absorption feature for higher densities. Thus, the silicate feature might not be modeled realistically for Class I objects with low envelope densities, but should be a good approximation for more typical, higher-density envelopes.

The inner disk radius is set by the star's magnetosphere, which channels the accretion flow onto the star, and is roughly at the corotation radius, at a few stellar radii from the stellar surface (Shu et al. 1994). However, in some models the inner disk radius was set equal to the stellar radius, implying a boundary layer. The accretion luminosity is set as the sum of the disk accretion luminosity, generated as matter accretes through the disk, and of the hot component luminosity, produced by the dissipation of the accretion shock on the stellar surface. For the cases where the inner disk radius is equal to the stellar radius, half of the accretion luminosity is emitted by the disk, and the other half by the boundary layer. For larger inner disk radii, the accretion shock accounts for a larger fraction of the accretion luminosity than matter accreting through the disk.

\subsubsection{Dust and Ices}

The dust in the envelope is composed of small silicate and graphite grains (Draine \& Lee 1984), troilite $(\mathrm{FeS})$, and water ice (Pollack et al. 1994), as well $\mathrm{CO}_{2}$ ice (Ehrenfreund et al. 1996). The particle size distribution follows a power law with particle radius $a: n(a) d a \propto$ $a^{-3.5} d a$, with maximum sizes of $0.3 \mu \mathrm{m}$. This maximum grain size corresponds to the typical value found in the interstellar medium (e.g., Mathis, Rumpl, \& Nordsieck 1977; Weingartner \& Draine 2001) and is motivated by the notion that envelopes likely contain pristine material. Our data confirm this idea, since the shape of the silicate absorption feature in most of our Class I objects indicates that the dust is dominated by small grains (see also Osorio et al. 2003). The abundance of the various components of the dust mixture is similar to the one adopted by Osorio et al. (2003), who adjusted the abundances given in Draine \& Lee (1984) and Pollack et al. (1994) to fit a model to the spectrum of L1551 IRS 5. We do not include organics in our mixture; we assume that the carbon is present in the form of graphite only. The fractional abundances with respect to the mass of the gas for silicates, graphite, troilite, and $\mathrm{H}_{2} \mathrm{O}$ ice are $\zeta_{\text {sil }}=0.004$, $\zeta_{\text {gra }}=0.0025, \zeta_{\text {tro }}=0.000768$, and $\zeta_{\text {ice }}=0.0005$, respectively. To get a better fit to the observed SEDs, these fractional mass abundances could be varied until a good match was found, but in order to limit the number of free parameters in the fit, we kept the abundances of these components constant. Only the $\mathrm{CO}_{2}$ ice abundance was adjusted for each object to reproduce the depth of the $\mathrm{CO}_{2}$ ice feature.

The opacities used in the current model should be regarded as approximations: the water ice opacities used in the model do not generate a feature at $6.0 \mu \mathrm{m}$, which is detected in our IRS spectra of Class I objects, and other ice opacities, e.g. for methanol ice, which might be responsible for the feature at $6.8 \mu \mathrm{m}$, are not included. Also, the $15.2 \mu \mathrm{m}$ ice feature is treated as pure $\mathrm{CO}_{2}$ ice, but in reality is likely mixed with water and other ices, making the feature broader (Ehrenfreund et al. 1999). Therefore, our envelope models are not able to reproduce the majority of the ice features, and the most prominent ice feature, the $\mathrm{CO}_{2}$ feature, can only be approximated in its depth, not its width. On the other hand, since the purpose of this study is a characterization of the overall SEDs of Class I objects, and thus of their envelope structure, we will neglect the fact that the ice features will not be reproduced well or at all.

\subsubsection{Model Parameters}

The input parameters for the models are the total system luminosity $\left(L_{s t a r}+L_{a c c}\right)$, the fraction of the total luminosity that arises from the star $\left(\eta_{\text {star }}\right)$, the radius of the star (assumed to be $2 \mathrm{R}_{\odot}$ ), the minimum disk radius, 
the centrifugal radius (which is essentially the outer disk radius), the inner and outer envelope radii, the flattening of the envelope (for sheet-collapse models), and the reference density $\rho_{1}$, which is the density the envelope would have at a radius of $1 \mathrm{AU}$ for the limit $R_{c}=0$. The latter parameter, $\rho_{1}$, is related to the mass infall rate $\dot{M}_{i}$ and the central mass $M_{*}$ (Kenvon. Calvet, \& Hartmann 1993a):

$\rho_{1}=5.3 \times 10^{-14}\left(\frac{\dot{M}_{i}}{10^{-5} M_{\odot} \mathrm{yr}^{-1}}\right)\left(\frac{M_{*}}{1 M_{\odot}}\right)^{-1 / 2} \mathrm{~g} \mathrm{~cm}^{-3}$

For a fixed central mass, $\rho_{1}$ determines the mass infall rate. The inner radius of the envelope is determined by the dust destruction, which, for typical dust made of silicates, is at the dust sublimation temperature of $\sim$ $1500 \mathrm{~K}$. If a cavity is included, the inner envelope radius will be farther out. The outer envelope radius is constrained particularly by sub-mm and mm observations, since this parameter affects the SED almost exclusively beyond about $100 \mu \mathrm{m}$; typical values are several 1000 AU.

For sheet-collapse models, the flattening of the envelope is described by $\eta=R_{\text {out }} / H$, where $R_{\text {out }}$ is the outer radius of the envelope and $H$ is the scale height of the original sheet, an isothermal, infinite, flat layer in hydrostatic equilibrium, and is defined by $H=c_{s}^{2} /(\pi G \Sigma)$, with $\Sigma$ equal to the surface density (Hartmann et al. 1994). Over the course of the collapse of a flattened molecular cloud core, it is thought that $\eta$ increases, reflecting an increase in the size of the cavity.

The emergent SED is computed with the disk radius (set equal to $10 \mathrm{AU}$ for the disk component, and to $R_{c}$ for the stellar and envelope components), the inclination angle to the line of sight, and the opening angle of the cavity as parameters. Scattering is also included, which causes an increase in flux at near-infrared wavelengths $(\lesssim 3 \mu \mathrm{m})$. The parameters that affect a modeled SED the most are $\rho_{1}, R_{c}$, and the inclination angle. The higher the density, the deeper the silicate absorption feature and the longer the wavelength at which the SED peaks. A high inclination angle will also increase the silicate feature depth, in addition to decreasing the emission below $8 \mu \mathrm{m}$ and increasing the emission in the far-infrared. The centrifugal radius, besides representing the outer disk radius, also defines the region where infalling material piles up; thus, for smaller $R_{c}$ high-density material can be found closer to the central protostar, resulting in more midto far-infrared emission and a deeper silicate absorption feature. A sketch of our model envelope, with important model parameters identified, is shown in Figure 4.

From previous studies (e.g., Kenvon et al. 1993b), typical values for young protostars in the Taurus star-forming region are an $R_{c}$ of $\sim 100 \mathrm{AU}$, and mass infall rates a few times $10^{-6} \mathrm{M}_{\odot} \mathrm{yr}^{-1}$. Observations exclude centrifugal radii larger than about $1000 \mathrm{AU}$ and mass infall rates larger than $10^{-6} \mathrm{M}_{\odot} \mathrm{yr}^{-1}$. From equation 1, it follows that typical values for $\rho_{1}$ are of the order of $10^{-14}$ to $10^{-13} \mathrm{~g} \mathrm{~cm}^{-3}$. Hartmann. Calvet, \& Boss (1996) find that characteristic values of $\eta$ are between 2.5 and 3.5 for the main infall phase of the envelope, resulting in large opening angles for the cavities. Over time, $\eta$ should increase, up to a maximum value of $\sim 4$, and the cavities become larger. Inclinations are usually larger than $60^{\circ}$, but this could be a selection effect, since the SEDs of low-inclination protostars with large cavities would resemble the SEDs of T Tauri stars and therefore not be classified as Class I objects (Kenvon et al. 1993b).

In the following section, we will introduce our envelope models for 22 Class I objects of our sample. In Figures 5 to 28 (excluding Figures 6 and 18), we show the SED of each object together with the best-fit model (determined by eye) and its components, the star (dotted gray line), the disk (dash-dotted gray line), both extinguished by the envelope, and the envelope (long-dashed gray line); the sum of all components is represented by the thick, solid, gray line. All model protostars were placed at a distance of $140 \mathrm{pc}$.

\subsection{Models of Individual Class I Objects \\ 4.2.1. $04016+2610$}

Padgett et al. (1999) imaged this object in the nearIR with HST; it is surrounded by a nebulosity on one side, with the protostar at its base. This might indicate that we are observing IRAS 04016+2610 through the opening of a large cavity in the envelope. A bipolar molecular outflow is oriented along the axis of symmetry of the nebula (e.g., Gomez et al. 1997; Hogerheijde et al. $1998)$; its inclination angle was estimated to be $60^{\circ}$ by Hogerheijde et al. (1998).

In Figure 5 we show an envelope model for IRAS $04016+2610$ whose parameters were adjusted to yield a good fit of the near-IR to $\mathrm{mm}$ SED. A sheet-collapse model with $\eta=1$ had to be adopted; TSC models did not yield enough flux on the short- and long-wavelength sides of the $10 \mu \mathrm{m}$ silicate feature. The IRS spectrum is fitted reasonably well between about 8.5 and $25 \mu \mathrm{m}$; the discrepancies at the shorter wavelength side might be resolved by adjusting the abundances of the dust and ices in the model, while the lower IRS fluxes beyond $25 \mu \mathrm{m}$ could be an aperture effect, with the IRS slits truncating the regions contributing to the $20-40 \mu \mathrm{m}$ region.

The model shown in Figure [5, with a density of $4.5 \times 10^{-14} \mathrm{~g} \mathrm{~cm}^{-3}$, an $R_{c}$ value of $100 \mathrm{AU}$, and an inclination angle of $40^{\circ}$, is one of several that yielded a comparable good fit. In particular, values for $\rho_{1}$ down to $3.5 \times 10^{-14} \mathrm{~g} \mathrm{~cm}^{-3}$ and for $R_{c}$ down to $70 \mathrm{AU}$, as well as inclination angles within $30^{\circ}-50^{\circ}$, would yield acceptable fits. The lower values for $\rho_{1}$ and $R_{c}$ would match previous modeling results of $\mathrm{KCH} 93$, who derived $3.2 \times 10^{-14}$ $\mathrm{g} \mathrm{cm}^{-3}$ and $70 \mathrm{AU}$, respectively, for these two parameters. They would also be roughly consistent with results from Whitnev et al. (1997), who derived an even smaller $R_{c}$ of $50 \mathrm{AU}$ and an inclination angle of $57^{\circ}$. On the other hand, our best-fit model agrees very well with the results of Eisner et al. (2005) and of Gramajo et al. (2007). A much smaller $R_{c}$ would result in an SED that is too narrow and contain too much flux in the $20-50 \mu \mathrm{m}$ region, while a large $R_{c}$ would require larger inclination angles to match the $10 \mu \mathrm{m}$ feature and longer-wavelength IRS flux, but it would also decrease the emission below $8 \mu \mathrm{m}$. Stark et al. (2006) require a larger centrifugal radius of 300 AU, combined with a larger value for $\rho_{1}$ and an inclination angle of $65^{\circ}$, to reproduce the near-IR images of IRAS $04016+2610$.

Our derived range for the inclination angle, $30^{\circ}-50^{\circ}$, is 
somewhat smaller than the value of $60^{\circ}$ derived from observations (Hogerheijde et al. 1998; Padgett et al. 1999) and modeling (KCH93). In addition, our derived cavity semi-opening angle of $5^{\circ}$ is smaller than expected from the images; for example, Stark et al. (2006) derive a value of $25^{\circ}$ for $\theta$. If we adopted a larger cavity, the emission from near-IR wavelengths to about $20 \mu \mathrm{m}$ would decrease and therefore not fit the shape of most of the IRS spectrum. Finally, our adopted luminosity of 4.5 $\mathrm{L}_{\odot}$ is larger than the bolometric luminosity of $3.7 \mathrm{~L}_{\odot}$ (KH95), but necessary to yield enough flux.

\subsection{2. $04108+2803 B$}

This protostar is part of a binary; it is separated by about $21^{\prime \prime}$ from component $\mathrm{A}$, which is less embedded, displays a silicate emission feature, but is about a factor of 5 fainter in the mid-infrared (see Figure 6). Therefore, component B clearly dominates in the mid-IR; while $04108+2803$ A probably is a reddened T Tauri star, $04108+2803$ B is a Class I object. Both sources have no optical counterparts (e.g., Tamura et al. 1991).

According to Young et al. (2003), IRAS 04108+2803 B is a Class I object based on its bolometric temperature of $179 \mathrm{~K}$, but would be classified as a Class 0 object based on its submillimeter luminosity. This IRAS source is not resolved in the 450 and $850 \mu \mathrm{m}$ maps of Young et al. (2003); since it is also very faint at $1.3 \mathrm{~mm}(39 \mathrm{mJy}$; Motte \& André 2001) and does not have an outflow, it is likely an evolved protostar with a smaller envelope.

The envelope model for IRAS $04108+2803$ B requires relatively low density $\left(\rho_{1}=1.5 \times 10^{-14} \mathrm{~g} \mathrm{~cm}^{-3}\right)$, a small centrifugal radius $\left(\mathrm{R}_{c}=40 \mathrm{AU}\right)$, a moderate-size cavity $\left(\theta=10^{\circ}\right)$, and an inclination angle of $40^{\circ}$ (Figure 7). The outer envelope radius was set at 6000 AU, which is larger than the projected binary separation of 3000 AU, but necessary to fit the sub-mm and $\mathrm{mm}$ emission. This suggests that component A is likely obscured by this envelope; alternatively, IRAS 04108+2803 B could be surrounded by a smaller envelope, but a fairly large circumstellar disk, which would add to the long-wavelength emission.

The IRS spectrum is fitted rather well with this envelope model; the change in slope of the spectrum beyond $25 \mu \mathrm{m}$ could be an effect of slit truncation. The fit of the $10 \mu \mathrm{m}$ feature and of the slope of the IRS spectrum between 10 and $25 \mu \mathrm{m}$ suggests that the values for the density and the centrifugal radius are relatively well-constrained. Previous models by KCH93 and also by Whitney et al. (1997) obtained higher values for $\rho_{1}$ and $R_{c}$ (about double our values), but with comparable inclination angles. Eisner et al. (2005) derived a large value for $\rho_{1}$, too, but an $R_{c}$ similar to our value; their best-fit model included a massive disk surrounded by a 500 AU envelope.

In our models, we can exclude $R_{c}$ values larger than about $50 \mathrm{AU}$, since they would result in a decrease in flux from 15 to $40 \mu \mathrm{m}$ due to an overall shallower SED shape. A reference density up to $2.0 \times 10^{-14} \mathrm{~g} \mathrm{~cm}^{-3}$ would still fit the observed SED, if combined with a smaller inclination angle $\left(\sim 25^{\circ}\right)$ to reduce the depth of the silicate absorption feature. The cavity semi-opening angle of $10^{\circ}$ is required to fit the IRS spectrum; a smaller opening angle would result in an increase in flux in the 10-25 $\mu \mathrm{m}$ wavelength range. The inner disk radius was set at 3 stellar radii to decrease the flux in the $2-8 \mu \mathrm{m}$ region; this parameter does not affect the remaining parts of the SED.

\subsection{3. $04154+2823$}

IRAS $04154+2823$ might be a transition object between the Class I and II stage, since it has an SED that is somewhat decreasing in the mid-IR, and it also does not display a prominent silicate absorption feature. However, its spectrum shows a clear $\mathrm{CO}_{2}$ ice absorption feature at $15.2 \mu \mathrm{m}$, and its overall infrared SED is relatively flat, suggesting that some envelope material should be present. It is also very faint in the optical (Strom \& Strom 1994), possibly indicating larger extinction along the line of sight. It is somewhat reminiscent of IRAS $04158+2805$, which is also faint and has a relatively flat SED over the IRS spectral range.

This protostar has a low luminosity $\left(\mathrm{L}_{b o l}=0.3-0.4\right.$ $\mathrm{L}_{\odot}$; see Table 4); its envelope model requires a very low reference density of $\rho_{1}=7.0 \times 10^{-15} \mathrm{~g} \mathrm{~cm}^{-3}$ and a very small centrifugal radius of $10 \mathrm{AU}$ to reproduce the midIR spectrum as well as the sub-mm measurements (Figure 8). However, this model overestimates the flux between 20 and $100 \mu \mathrm{m}$ and slightly underestimates the mm flux. A luminosity value higher than about $0.4 \mathrm{~L}_{\odot}$ would result in an increase in the long-wavelength flux, which would fit the $\mathrm{mm}$ data better, but yield even more flux between 20 and $60 \mu \mathrm{m}$. An increase in $\rho_{1}$ would have a similar effect; in addition, values of $\rho_{1}$ larger than about $8.0 \times 10^{-15} \mathrm{~g} \mathrm{~cm}^{-3}$ can be excluded given the constraint of the weak silicate absorption feature. The low inclination angle $\left(i=20^{\circ}\right)$ is also well-constrained by the $10 \mu \mathrm{m}$ feature and the shape of the SED.

\subsection{4. $04158+2805$}

According to Ménard et al. (2001) who imaged this source in the optical, IRAS $04158+2805$ is likely surrounded by an edge-on disk and a bipolar nebula; a jet is oriented perpendicular to the disk plane, in a geometry similar to HH 30. Park \& Kenvon (2002) observed that this object is not extended in the near-IR or at millimeter continuum wavelengths, and therefore it could be a heavily reddened T Tauri star and not a Class I object. Recently, Andrews \& Williams (2007) modeled the SED of IRAS $04158+2805$ with only a large disk component. However, Kenvon et al. (1998) concluded from their optical spectra and optical and near-IR photometry that IRAS $04158+2805$ is a Class I source. Unpublished HST images at optical and near-IR wavelengths (programs 9103 and 10603, PI K. Stapelfeldt and D. Padgett, respectively) show an extended, one-sided conical nebulosity, confirming that this source is surrounded by an envelope. We note that this object is of a late spectral type (M5-M6 according to Luhman 2006; M6 according to White \& Hillenbrand 2004); it will probably become a very low-mass star, close to the hydrogen-burning mass limit.

The SED of IRAS $04158+2805$ can be fitted with an envelope model with relatively low density $\left(\rho_{1}=\right.$ $\left.2.0 \times 10^{-14} \mathrm{~g} \mathrm{~cm}^{-3}\right)$, small centrifugal radius $\left(\mathrm{R}_{c}=60\right.$ $\mathrm{AU})$, small cavity $\left(\theta=5^{\circ}\right)$ and an inclination angle of $30^{\circ}$ (Figure 9). The inner disk radius was set at 3 stellar radii to decrease the amount of flux emitted at near-IR 
and mid-IR wavelengths out to $8 \mu \mathrm{m}$. The outer envelope radius was set at $5000 \mathrm{AU}$ to somewhat decrease the emission in the sub-mm and mm. Nevertheless, the model overestimates the flux at wavelengths longer than $30 \mu \mathrm{m}$, but lies in between the two measurements around $1 \mathrm{~mm}$. The discrepancy between the two long-wavelength data points could be explained by the large difference of the two beam sizes (see Table 2); the $880 \mu \mathrm{m}$ measurement likely misses some extended emission.

We adopted a luminosity of $0.3 \mathrm{~L}_{\odot}$, which is $50 \%$ larger than the bolometric luminosity we measured by integrating the fluxes fluxes of the SED; a model with $L=0.2$ $\mathrm{L}_{\odot}$, a smaller density $\left(\sim 1.0 \times 10^{-14} \mathrm{~g} \mathrm{~cm}^{-3}\right)$, and a centrifugal radius around 10 AU would yield a comparable fit, but still overestimate the emission starting at $20 \mu \mathrm{m}$. Overall, the model parameters are mainly constrained by the IRS spectrum; the depth of the silicate feature and the shape of the IRS spectrum from 12 to $30 \mu \mathrm{m}$ constrain the reference density $\rho_{1}$ and the cavity semi-opening angle, respectively, reasonably well. With the current reference density, a much smaller centrifugal radius would result in even higher fluxes in the 30-60 $\mu \mathrm{m}$ region, while a value larger than about $90 \mathrm{AU}$ would decrease emission from about 15 to $40 \mu \mathrm{m}$. A $\rho_{1}$ value of about $1.5 \times 10^{-14} \mathrm{~g} \mathrm{~cm}^{-3}$, combined with an $R_{c}$ value of $50 \mathrm{AU}$ and a slightly larger inclination angle of $40^{\circ}$ would also yield an acceptable fit. We can exclude an edge-on orientation based on the shape of the IRS spectrum. Only the availability of accurate, far-IR to $\mathrm{mm}$ fluxes will improve the model fit of IRAS $04158+2805$.

\subsection{5. $04166+2706$}

This IRAS source is very faint in the near-IR; no source is detected in 2MASS, but both Kenvon et al. (1990) and Park \& Kenyon (2002) detected a faint near-IR source close to the IRAS position, which we adopted for the pointing of our IRS observations. The latter authors describe the appearance of IRAS $04166+2706$ as low surface brightness nebulosity. Motte \& André (2001) and Tafalla et al. (2004) detected a dense, round core at $\mathrm{mm}$ wavelengths coinciding with the IRAS source. Thus, this Class I object is likely deeply embedded. It also has bipolar outflows (Bontemps et al. 1996), which are highly collimated and of extremely high velocity (Tafalla et al. 2004), consistent with this object's early evolutionary stage, possibly placing it at the Class 0 stage. The high degree of symmetry of the red- and blueshifted gas of the outflows (Tafalla et al. 2004) suggests that this object is likely seen close to edge-on. For the SED plot, we included the $\mathrm{H}$ and $\mathrm{K}$ magnitudes measured by Kenvon et al. (1990).

The SED shape of IRAS $04166+2706$ further indicates that it is likely seen at a large inclination angle, close to edge-on. In fact, an envelope model that fits it well (see Figure 10) has an inclination angle of $85^{\circ}$. Smaller inclination angles $\left(70^{\circ}-80^{\circ}\right)$ would result in models that match the observed spectrum from 15 to $30 \mu \mathrm{m}$ more closely, but they would overestimate the emission from the near-IR to $8 \mu \mathrm{m}$. Increasing the cavity semi-opening angle by just $1^{\circ}$ from its current value of $6^{\circ}$ would lower the flux between 8 and $30 \mu \mathrm{m}$, resulting in a better fit of the slope of the spectrum beyond $12 \mu \mathrm{m}$, but a poorer fits of the silicate feature. An inner disk radius of 5 stellar radii was adopted to lower the flux below $8 \mu \mathrm{m}$.
A reference density of $4.5 \times 10^{-14} \mathrm{~g} \mathrm{~cm}^{-3}$ and a large centrifugal radius $\left(R_{c}=300 \mathrm{AU}\right)$ are required to fit the depth of the silicate feature and also the width of the SED. Values of $\rho_{1}$ and $R_{c}$ down to $3.5 \times 10^{-14} \mathrm{~g} \mathrm{~cm}^{-3}$ and $200 \mathrm{AU}$, respectively, would also yield good fits; only the silicate feature would be somewhat narrower. The best-fitting model of KCH93, who had fewer constraints available, resulted in $\rho_{1}=1.0 \times 10^{-13} \mathrm{~g} \mathrm{~cm}^{-3}, R_{c}=70$ AU, and $i=30^{\circ}$; a high-inclination model with the same $R_{c}$, but $i=90^{\circ}$ and $\rho_{1}=3.2 \times 10^{-14} \mathrm{~g} \mathrm{~cm}^{-3}$ was also suggested as a fit. This underlines the importance of the IRS spectrum to discriminate between different models.

We note that our model does not fit the 850 and 1300 $\mu \mathrm{m}$ measurements, which were done with a larger beam size (see Table 2), but it fits the data taken at similar wavelengths, but with $\sim 20^{\prime \prime}$ apertures. This could indicate that some extended, cold dust outside of the infall region contributes to the long-wavelength emission (see also Javawardhana et al. 2001).

\subsection{6. $04169+2702$}

This object, which is not detected at optical wavelengths, is surrounded by a cometary reflection nebula in the near-IR and associated with Herbig-Haro emission knots (Tamura et al. 1991; Gomez et al. 1997). It also has bipolar outflows (e.g., Moriarty-Schieven et al. 1992; Bontemps et al. 1996), which are perpendicular to an elongated envelope structure, about $2200 \mathrm{AU} \times 1100$ $\mathrm{AU}$ in size and inclined at $60^{\circ}$ from the line of sight (Ohashi et al. 1997b). Measurements of continuum emission at $2.7 \mathrm{~mm}$ did not resolve the source on scales of $2^{\prime \prime}-3^{\prime \prime}$, probably indicating that this emission arises from a disk of radius $\lesssim 150$ AU (Ohashi et al. 1997b).

The SED of IRAS $04169+2702$ is fitted well by a sheetcollapse envelope model $(\eta=1)$ with moderate density $\left(\rho_{1}=3.2 \times 10^{-14} \mathrm{~g} \mathrm{~cm}^{-3}\right)$, a centrifugal radius of $100 \mathrm{AU}$, basically no cavity and a high inclination angle (Figure 11). Compared to the results obtained by KCH93, both our reference density and centrifugal radii are smaller by about a factor 3 , and our inclination angle is larger $\left(75^{\circ}\right.$ versus $\left.30^{\circ}\right)$. Whitney et al. (1997) modeled this object with an $R_{c}$ of just $10 \mathrm{AU}$, a reference density half our value, a cavity semi-opening angle of $11^{\circ}$, and an inclination angle of $45^{\circ}$. However, our model is better constrained. Both $\eta$ and the cavity semi-opening angle are determined by the shape of the IRS spectrum beyond about $12 \mu \mathrm{m}$. In particular, a TSC model would yield less flux from about 10 to $100 \mu \mathrm{m}$ given the same envelope parameters. The large $R_{c}$ value is required to fit the long-wavelength part of the IRS spectrum, while the value for the reference density is constrained by the shape of the silicate absorption feature and the peak of SED. The $10 \mu \mathrm{m}$ feature and the SED shape also constrain the high inclination angle, which has to be larger than $70^{\circ}$ to yield a good fit.

The luminosity used for the model $\left(L=1.5 \mathrm{~L}_{\odot}\right)$ is twice as high as the bolometric luminosity of $0.8 \mathrm{~L}_{\odot}$ measured by KH95, but close to the value we calculated by integrating the fluxes of the SED, $1.2 \mathrm{~L}_{\odot}$, and to the value of $1.4 \mathrm{~L}_{\odot}$ determined by Kenvon et al. (1990). We can exclude a lower luminosity from our model fit, since it would not yield enough emission to match the observations. As for IRAS $04166+2706$, our model does not fit the long-wavelength emission measured with large aper- 
ture sizes, but it matches the observations taken with smaller apertures. Again, we suggest that some cold dust just outside the infalling envelope is responsible for the extended mm emission.

\subsection{7. $04181+2654 A$}

This IRAS source is a member of a wide binary system; component $\mathrm{B}$ lies to the northwest of component A, at a projected distance of $31^{\prime \prime}$, which corresponds to about $4300 \mathrm{AU}$ at the distance of Taurus. In addition, the IRAS source $04181+2655$ lies somewhat less than $1^{\prime}$ to the northwest of $04181+2654 \mathrm{~B}$. A bipolar outflow is centered on IRAS $04181+2655$, but no outflow has been detected from the IRAS sources to the south (Bontemps et al. 1996), which could be the result of a low inclination angle along the line of sight of these two objects.

Of the two components of the $04181+2654$ binary, the B component seems to be more deeply embedded, since it is fainter than A by a factor of 12 in the $J$ band, but only by about a factor of 2 in the $K$ band. Over the mid-IR spectral range, component $B$ is fainter than component A by a factor of 2 to 3 . The silicate feature of IRAS $04181+2654$ B is seen partly in self-absorption, indicating that it is oriented along the line of sight such that part of the silicate emission originating in the inner disk is absorbed in the envelope.

Motte \& André (2001) measured the $1.3 \mathrm{~mm}$ flux of IRAS $04181+2654 \mathrm{~A}$, which is dominated by emission from an extended envelope. According to Park \& Kenyon (2002), both A and B are extended at $\mathrm{mm}$ continuum wavelengths, but not in the near-IR. They suggest that both sources are protostars; therefore, they are likely surrounded by an envelope which could be truncated in its outer parts. Images at $0.9 \mu \mathrm{m}$ obtained by Eisner et al. (2005) reveal a faint nebulosity around IRAS $04181+2654 \mathrm{~A}$, possibly tracing scattered light from an envelope cavity; the $\mathrm{B}$ component is not detected.

The SED of IRAS $04181+2654$ A can be fitted by an envelope model with a reference density of $2.0 \times 10^{-14}$ $\mathrm{g} \mathrm{cm}^{-3}$, a centrifugal radius of $50 \mathrm{AU}$, a cavity semiopening angle of $7^{\circ}$, and an inclination angle of $20^{\circ}$ (Figure 12). In order to fit the SED shape from the near-IR to $8 \mu \mathrm{m}$, we set the inner disk radius to 5 stellar radii; even with this value, the near-IR fluxes are somewhat underestimated. We chose an outer envelope radius of 10000 AU; such a large envelope would encompass component $\mathrm{B}$, too, but is necessary to yield enough flux in the $\mathrm{mm}$ wavelength range. The fact that the model still underpredicts the flux at $1.3 \mathrm{~mm}$ suggests that the $\mathrm{mm}$ measurement probably includes some contribution from an envelope around component B.

The density is constrained by the depth of the silicate absorption feature, which is rather shallow; it could be slightly larger, but then require a somewhat smaller cavity semi-opening angle to decrease emission in the 8$25 \mu \mathrm{m}$ range. The slope of the spectrum from 12 to $30 \mu \mathrm{m}$ constrains both the opening angle of the cavity and the value of $R_{c}$. A larger $R_{c}$ would result in a broader SED, but at the same time decrease the flux levels in the long-wavelength part of the IRS spectrum. A smaller $R_{c}$ would generate a steep rise of the flux levels beyond $12 \mu \mathrm{m}$. KCH93 modeled both IRAS 04181+2654
$\mathrm{A}$ and $\mathrm{B}$ with an envelope with a higher reference density, $3.2 \times 10^{-14} \mathrm{~g} \mathrm{~cm}^{-3}$, a somewhat larger $R_{c}(70 \mathrm{AU}$ versus our $50 \mathrm{AU})$, and a comparable inclination angle. Whitnev et al. (1997) used the same value for $R_{c}$ as we did in our model, but with almost double the values for reference density and inclination angle.

Overall, the model for IRAS $04181+2654 \mathrm{~A}$ is poorly constrained, given the lack of sub-mm data points and the uncertainty of the $1.3 \mathrm{~mm}$ measurement due to the presence of component B. We did not model that component, since there are no sub-mm or mm measurements available that would constrain the long-wavelength part of the SED.

\subsection{8. $04239+2436$}

In the near-infrared, a symmetric reflection nebula surrounds this IRAS source, which is a binary separated by 0.3 (Reipurth et al. 2000). The brighter component (at $2.2 \mu \mathrm{m}$ ) of this system drives a large, asymmetric HerbigHaro jet, HH 300 (Reipurth et al. 2000); the asymmetry suggest that the inclination angle of this object is closer to face-on than edge-on. Maps of this Class I object at $450,850,1100$, and $1300 \mu \mathrm{m}$ show that it is a point source with some diffuse emission from circumstellar material (Chini et al. 2001; Eisner et al. 2005). Therefore, IRAS $04239+2436$ is extended from infrared to millimeter wavelengths (see also Park \& Kenyon 2002), typical for Class I objects.

The SED of IRAS $04239+2436$ requires a model with relatively low density $\left(\rho_{1}=1.3 \times 10^{-14} \mathrm{~g} \mathrm{~cm}^{-3}\right)$ and very small centrifugal radius $\left(R_{c}=10 \mathrm{AU}\right)$ (Figure 13). A higher density would increase the depth of the silicate feature, while a larger centrifugal radius would decrease the emission in the 10 to $40 \mu \mathrm{m}$ region and result in a shallow silicate absorption feature. Increasing $\rho_{1}$ and $R_{c}$ up to about $2.0 \times 10^{-14} \mathrm{~g} \mathrm{~cm}^{-3}$ and $20 \mathrm{AU}$, respectively, would still result in good fits if the luminosity was increased by about $20 \%$.

Previous models by KCH93 yielded a larger density, $3.2 \times 10^{-14} \mathrm{~g} \mathrm{~cm}^{-3}$, and a centrifugal radius of $70 \mathrm{AU}$, while models by Whitney et al. (1997) resulted in values for $\rho_{1}$ and $R_{c}$ very similar to ours. Eisner et al. (2005) derived a reference density almost twice our value and a centrifugal radius of $30 \mathrm{AU}$; more recent results by Gramaio et al. (2007) found the same centrifugal radius as Eisner et al. (2005), but a reference density similar to ours. Our inclination angle of $15^{\circ}$ is smaller than the values derived by previous modeling efforts, which range from $30^{\circ}$ to $63^{\circ}$; a value of up to $i=30^{\circ}$ would yield an acceptable fit, albeit with somewhat decreased emission from the near-IR to about $25 \mu \mathrm{m}$. The low inclination angle is well-constrained by the SED, and it is also consistent with the near-IR morphology and observed asymmetry of the Herbig-Haro jet (see, e.g., Reipurth et al. 2000).

With the current values for $R_{c}$ and $\rho_{1}$, the model does not reproduce the shape of the silicate absorption feature very well, especially at the long-wavelength side; this could be an effect of dust and ice composition, since the overall width of the SED and shape of the IRS spectrum beyond $15 \mu \mathrm{m}$ suggest such a small $R_{c}$ value.

4.2.9. $04248+2612$ 
This IRAS source is actually a subarcsecond binary whose components are roughly similar in brightness. Its IRS spectrum shows a silicate emission feature and some very weak ice absorption features. In the near-IR, it is surrounded by a bipolar reflection nebula with a somewhat complex morphology, but likely seen close to edge-on (Padgett et al. 1999). This object is also somewhat extended at 450 and $850 \mu \mathrm{m}$; the extension parallel to the bipolar nebulosity seen in the near-IR is likely tracing dust heated in an outflow (Young et al. 2003). White \& Hillenbrand (2004) note that IRAS $04248+2612$ is the lowest-mass protostar known that drives a molecular outflow and an $\mathrm{HH}$ object (Moriarty-Schieven et al. 1992; Gomez et al. 1997); they suggest it could actually be a substellar Class I object. However, a recent spectral type determination of M4M5 for this object (Luhman 2006) suggests that it will probably evolve into a very low-mass star.

Since this Class I object displays a silicate emission feature, and in the near-IR the central binary is detected, its envelope likely has low densities. Our model suggests a reference density of $4.0 \times 10^{-15} \mathrm{~g} \mathrm{~cm}^{-3}$, which is the lowest $\rho_{1}$ value among the protostars modeled here, indicating an advanced stage of evolution for this protostar. Our model further includes a relatively small centrifugal radius of $30 \mathrm{AU}$, a cavity semi-opening angle of $15^{\circ}$, and an inclination angle of $70^{\circ}$ (see Figure 14). IRAS $04248+2612$ is similar to $\mathrm{CoKu}$ Tau/1, which is also seen close to edge-on through a low-density envelope. The short-wavelength emission is fitted very well; it is dominated by the stellar component, which agrees with the fact that the central binary is detected at near-IR wavelengths. The emission from far-IR to $\mathrm{mm}$ wavelengths is underestimated; however, we found no set of parameters that could reproduce both the mid-IR and longwavelength parts of the SED. Thus, it is likely that the sub-mm and $\mathrm{mm}$ emission is generated by an additional component, probably by dust in a very extended region of cold dust outside the infall zone (Javawardhana et al. 2001).

Compared to modeling results by $\mathrm{KCH} 93$, who obtained two models of IRAS $04248+2612$, one with $\rho_{1}=1.0 \times 10^{-13} \mathrm{~g} \mathrm{~cm}^{-3}$ and $R_{c}=300 \mathrm{AU}$, and one with $\rho_{1}=3.2 \times 10^{-14} \mathrm{~g} \mathrm{~cm}^{-3}$ and $R_{c}=70 \mathrm{AU}$, our model has a clearly lower density (by a factor of 8 ) and a smaller centrifugal radius than the lower values of KCH93. Also models by Whitney et al. (1997) resulted in a larger centrifugal radius $(100 \mathrm{AU})$ and reference density $(1.5 \times$ $10^{-14} \mathrm{~g} \mathrm{~cm}^{-3}$ ) than our values; however, they derived a large inclination angle, as we did with our model. Recent modeling efforts by Stark et al. (2006) yielded a model very similar to ours.

Based on our fit to the IRS spectrum, especially the presence of a silicate emission feature, we can exclude a much higher density, and the fit to the shape of the IRS spectrum between 15 and $25 \mu \mathrm{m}$ also constrains the centrifugal radius to lie around 30 AU. Comparable model fits could be achieved with $\rho_{1}$ values in the (3.0-5.0) $\times 10^{-15} \mathrm{~g} \mathrm{~cm}^{-3}$ range and $R_{c}$ values between 20 and $40 \mathrm{AU}$, with smaller reference densities accompanied by smaller centrifugal radii. Our inclination angle of $70^{\circ}$ not only matches two previously published models of this object (Whitney et al. 1997; Stark et al. 2006), but is also in good agreement with the near-IR morphology observed by Padgett et al. (1999).

\subsubsection{0. $04264+2433$}

The IRS spectrum of this IRAS source shows a prominent silicate emission feature, but also possibly some ice features in the 5-8 $\mu \mathrm{m}$ wavelength region and an overall rising SED in the mid-IR. Therefore, it is probably a protostar surrounded by envelope material. This notion is further supported by the fact that IRAS $04264+2433$ is surrounded by a reflection nebulosity and likely emitting a small bipolar HH jet (Devine et al. 1999).

Similar to IRAS $04248+2612$ and CoKu Tau/1, the detection of a silicate emission feature in this protostar indicates that the envelope has low densities and is thus likely at an advanced evolutionary stage. We can reproduce the SED of IRAS $04264+2433$ fairly well with a model whose reference density is $5.0 \times 10^{-15} \mathrm{~g} \mathrm{~cm}^{-3}$; in addition, the shape of the IRS spectrum between about 13 and $20 \mu \mathrm{m}$ constrains the value of the centrifugal radius, found to be $30 \mathrm{AU}$ (see Figure 15). The inclination angle of $87^{\circ}$ and cavity semi-opening angle of $13^{\circ}$ were fine-tuned to yield a good fit over the $5-20 \mu \mathrm{m}$ range. The model overestimates the emission in the near-IR somewhat, which can be mostly be attributed to the envelope component.

A previous model of IRAS $04264+2433$ by KCH93 indicated a density twice as high as our value, a centrifugal radius of only $10 \mathrm{AU}$, and an inclination angle of $60^{\circ}$. Despite the differences, the earlier model already gauged the properties of this object, namely a low-density, highly inclined envelope with a small disk. On the other hand, a model by Whitney et al. (1997) yielded different model parameters: a centrifugal radius of $50 \mathrm{AU}$, a reference density of $3.75 \times 10^{-14} \mathrm{~g} \mathrm{~cm}^{-3}$, and an inclination angle of $32^{\circ}$; only their cavity semi-opening angle is similar to our value. Our model would yield a comparable fit with a centrifugal radius of 20-40 AU, but not with smaller or larger values for $\rho_{1}$.

In order to get enough flux from the far-IR to the $\mathrm{mm}$ wavelength range, we had to increase the luminosity by a factor of 2 compared to the bolometric luminosity of 0.37 $\mathrm{L}_{\odot}$ measured by KH95. On the other hand, integrating the measured fluxes of the SED yields a bolometric luminosity of $0.5 \mathrm{~L}_{\odot}$; given the high inclination angle of this object, the luminosity determined in this way is likely an underestimate of the true value.

\subsubsection{1. $04295+2251$}

This object, also known as L1536 IRS, is relatively faint; based on its bolometric temperature of $270 \mathrm{~K}$, it is classified as a Class I object, but its large sub-mm luminosity would suggest it is a Class 0 object (Young et al. 2003). Given that IRAS $04295+2251$ seemed not to be extended in the near-IR or mm, Park \& Kenyon (2002) indicated that it could be an edge-on disk; however, data obtained by Eisner et al. (2005) shows that IRAS $04295+2251$ is somewhat extended at $1.3 \mathrm{~mm}$ and also at $0.9 \mu \mathrm{m}$, where scattered light is detected. In addition, Moriarty-Schieven et al. (1992) detected an outflow from this source, implying an early evolutionary stage for this object.

Our envelope model for IRAS $04295+2251$ reproduces the general shape of the SED (see Figure [16), but it 
does not represent a good fit to the $10 \mu \mathrm{m}$ silicate feature and somewhat underestimates the near-IR and $\mathrm{mm}$ emission. However, at the longer wavelengths, our model reproduces those fluxes measured with smaller apertures, $10^{\prime \prime}-20^{\prime \prime}$ in size (see Table 2); this suggests that a region of cold dust outside of the infall region could be present.

A luminosity of $0.8 \mathrm{~L}_{\odot}$ was necessary to obtain more emission especially at longer wavelengths, even though the source's bolometric luminosity is $0.5 \mathrm{~L}_{\odot}$ (determined from integrating the fluxes of the SED). In the literature, $\mathrm{L}_{b o l}$ values vary from $0.44 \mathrm{~L}_{\odot}(\mathrm{KH} 95)$ to $0.64 \mathrm{~L}_{\odot}$ (Myers et al. 1987). A small centrifugal radius of $20 \mathrm{AU}$ was adopted to reproduce the steep rise of the SED past $15 \mu \mathrm{m}$; a value larger than $30 \mathrm{AU}$ would result in a flatter SED in this wavelength region, while a smaller $R_{c}$ would decrease the near-IR emission and cause an even larger overestimate of the far-IR flux. A reference density up to $30 \%$ larger than our adopted value of $8.0 \times 10^{-15} \mathrm{~g}$ $\mathrm{cm}^{-3}$ would still yield an acceptable fit, but require lower inclination angles $\left(40^{\circ}-50^{\circ}\right)$ to generate only a shallow silicate absorption at $10 \mu \mathrm{m}$; these models would overestimate the emission between 3 and $10 \mu \mathrm{m}$ and in the far-IR.

A previous model of IRAS $04295+2251$ by KCH93 resulted in a larger centrifugal radius, $70 \mathrm{AU}$, and a smaller inclination angle, $i=30^{\circ}$, but in a comparable reference density. We can exclude an inclination angle smaller than about $60^{\circ}$ with the current model parameters, since it would increase the emission in the $2-8 \mu \mathrm{m}$ range and at far-IR wavelengths, where the model already overestimates the observed emission. Whitney et al. (1997) modeled this object with very different model parameters, including a larger centrifugal radius and reference density, and an inclination angle of only $18^{\circ}$. Eisner et al. (2005) used the same value for $\rho_{1}, 3.75 \times 10^{-14} \mathrm{~g} \mathrm{~cm}^{-3}$, and a similar inclination angle as Whitney et al. (1997), but an $R_{c}$ of $30 \mathrm{AU}$, which is comparable to our result.

The relatively low reference density, combined with the inclination of $70^{\circ}$ to the line of sight and a cavity semiopening angle of $5^{\circ}$, results in a self-absorbed silicate feature at $10 \mu \mathrm{m}$, with part absorption, part emission characteristics. However, our model does not reproduce the detailed shape of the observed $10 \mu \mathrm{m}$ feature. This is likely due to the fact that the disk component used in the model does not include the optically thin disk atmosphere that would generate a $10 \mu \mathrm{m}$ silicate emission feature, which would then be subject to absorption by dust in the envelope. In the current model, the disk component only contributes a silicate absorption feature (i.e., continuum extinguished by the envelope), while the dust in the envelope is responsible for the emission component at $10 \mu \mathrm{m}$ (see the dash-dotted and long-dashed lines, respectively, in Figure 16). Also the shape of the inner cavity could affect the depth of the silicate feature, for example by resulting in a hotter inner region for a given $\rho_{1}$. It is likely that only a more complex model will yield a better fit, considering that this object has a relatively low-density envelope and compact morphology, but large sub-mm luminosity.

\subsubsection{2. $04302+2247$}

In the near-IR, this object is surrounded by two bright, symmetric nebulae separated by a dark lane
(Padgett et al. 1999). Models of the very symmetric quadrupolar structure suggest that IRAS $04302+2247$ is seen almost exactly at an inclination angle of $90^{\circ}$ (Lucas \& Roche 1997; Wolf. Padgett. \& Stapelfeldt 2003). The central source is likely obscured by a circumstellar disk, and the near-IR nebulosity, seen in scattered light, traces the walls of a bipolar cavity in the envelope (Lucas \& Roche 1997; Wolf, Padgett, \& Stapelfeldt 2003). Outflows oriented perpendicular to the dark lane were detected from this object (Moriarty-Schieven et al. 1992; Lucas \& Roche 1998). The edge-on orientation of IRAS $04302+2247$ may explain why it is invisible in the optical and very faint in the mid-IR. The SED of this object peaks close to $100 \mu \mathrm{m}$ and shows a pronounced 10 $\mu \mathrm{m}$ absorption feature.

The SED of IRAS $04302+2247$ can be fitted with an envelope model with an inclination angle of $89^{\circ}$, moderate density $\left(\rho_{1}=3.0 \times 10^{-14} \mathrm{~g} \mathrm{~cm}^{-3}\right)$, and a centrifugal radius of $300 \mathrm{AU}$ (Figure 17). The deep silicate absorption feature and the steep slope of the IRS spectrum beyond $10 \mu \mathrm{m}$ require a high inclination angle, consistent with the near-IR images. A higher density would also increase the depth of the silicate feature, but it would decrease flux levels over the $2-50 \mu \mathrm{m}$ region, too. The semi-opening angle of the cavity is relatively wide $\left(22^{\circ}\right)$, also roughly consistent with the results of Padgett et al. (1999). The shape of the IRS spectrum beyond $12 \mu \mathrm{m}$, and the width of the SED, constrain the value of $R_{c}$, which has to be large. Previous models by $\mathrm{KCH} 93$, which had much fewer constraints, derived a centrifugal radius of only $70 \mathrm{AU}$, but a density about a factor of 3 higher than in our model, and an inclination angle of $60^{\circ}$. Whitney et al. (1997) found an even smaller value for $R_{c}$, $10 \mathrm{AU}$, and a reference density half as large as our value, but their results for cavity and inclination angles agree with ours. Recent models by Stark et al. (2006) resulted in very similar parameters as for our model, except for a somewhat smaller value for $\rho_{1}$.

The model emission is somewhat above the IRAS data points at 60 and $100 \mu \mathrm{m}$; this is likely due to the fact that our adopted luminosity of $1 \mathrm{~L}_{\odot}$ is higher than the observed bolometric luminosity of $0.34 \mathrm{~L}_{\odot}(\mathrm{KH} 95)$, which was determined from fewer flux measurements $(\lambda \leq 100$ $\mu \mathrm{m})$. Our model indicates that our adopted luminosity might be slightly higher than the actual source luminosity.

\subsubsection{3. $04325+2402$}

This protostar is surrounded by a bipolar reflection nebula that is seen in scattered light in the near-IR and shows a complex morphology (Hartmann et al. 1999). It is likely a multiple system, consisting of a central, subarcsecond binary and a faint companion about $8^{\prime \prime}$ away; both the binary and the companion are surrounded by an accretion disk and are accreting from their envelopes (Hartmann et al. 1999). The subarcsecond binary, which instead could just be the result of scattering off a complex structure around a single star, is the likely source of the reflection nebula detected in the near-IR (Hartmann et al. 1999). This object is also associated with a molecular outflow (Moriarty-Schieven et al. 1992; Hogerheiide et al. 1998).

Our IRS observations were centered on the central source (see Figure [18). Even though the SL slit was 
oriented such that it also included the companion about $8^{\prime \prime}$ away, this component did not enter our extraction window, and we also did not detect it separately; this is expected, since it is faint and likely seen edge-on (Hartmann et al. 1999), and even the spectrum of the bright central source is only about $40 \mathrm{mJy}$ between 5 and $10 \mu \mathrm{m}$, with a steeply rising continuum beyond. In LL, which is oriented close to perpendicular to SL, only the central source was included.

An envelope model that fits this object well requires a sheet-collapse model $(\eta=1.0)$, moderate density $\left(\rho_{1}=3.0 \times 10^{-14} \mathrm{~g} \mathrm{~cm}^{-3}\right)$, a centrifugal radius of 100 AU, a cavity semi-opening angle of $15^{\circ}$, and an inclination angle of $80^{\circ}$ (Figure 19). Choosing a sheet-collapse model with $\eta=1.0$ was necessary to fit the IRS spectrum in the $15-30 \mu \mathrm{m}$ range. The inclination angle is larger than the value of $\sim 60^{\circ}$ inferred from the orientation of the outflow by Hogerheijde et al. (1998), but the shape of the IRS spectrum requires a high-inclination model. A previous model by KCH93 also determined $i=60^{\circ}$, but a centrifugal radius and a reference density three times as large as the values derived here. Models by Whitney et al. (1997) resulted in an $R_{c}$ value of only $50 \mathrm{AU}$, but in a reference density only somewhat larger than our value, and very similar values for cavity and inclination angles $\left(11^{\circ}\right.$ and $81^{\circ}$, respectively). Compared to previous models, the IRS spectrum provides better constraints; a larger $R_{c}$ would result in a flatter midIR SED, while a larger density would generate a deeper silicate absorption feature.

The silicate feature is not well fitted with this model; it seems to be in absorption on the short-wavelength side, but with an emission component on the long-wavelength side. The binary nature of IRAS $04325+2402$ could account for the peculiar shape of the silicate feature, with one component possibly responsible for some silicate emission.

\subsubsection{4. $04361+2547$}

This Class I object, also known as TMR 1, is deeply embedded in its dense environment and surrounded by a large reflection nebula, about $30^{\prime \prime}$ in size (Tamura et al. 1991). It is a close binary with a separation of $0^{\prime \prime} .31$ (Terebey et al. 1998); the "companion" detected at a projected separation of $10^{\prime \prime}$ from this protostar, at the end of a nebulosity extending from the source to the southeast, is likely a background star (Terebey et al. 2000). IRAS $04361+2547$ is extended in the nearIR, mid-IR and mm continuum (Motte \& André 2001; Park \& Kenyon 2002; Luhman et al. 2006). An outflow has been detected from this object, but its morphology is not clearly bipolar, suggesting an inclination angle of about $60^{\circ}$ (Hogerheijde et al. 1998).

The steeply rising SED of IRAS 04361+2547 indicates that this object is seen at a high inclination angle (Figure 201). A good envelope model fit requires a sheetcollapse model $(\eta=1.5)$, an inclination angle of $80^{\circ}$, a cavity semi-opening angle of $15^{\circ}$, an $R_{c}$ value of 100 $\mathrm{AU}$, and a fairly typical density $\left(\rho_{1}=2.0 \times 10^{-14} \mathrm{~g}\right.$ $\mathrm{cm}^{-3}$ ). Modeling by KCH93 resulted in a reference density of $3.2 \times 10^{-14} \mathrm{~g} \mathrm{~cm}^{-3}$ and a centrifugal radius of only 10 AU. Also, while our model suggests a close to edge-on orientation, KCH93 derived an inclination angle of only $30^{\circ}$. Later models by Whitney et al. (1997) and Gramajo et al. (2007) confirm our result of a large inclination angle (and also our cavity opening angle), but they derive centrifugal radii about half as large as our value, and generally a somewhat larger reference density.

Our adopted reference density is required to fit the SED beyond $50 \mu \mathrm{m}$, as well as to produce a silicate feature at $10 \mu \mathrm{m}$ that is mostly dominated by an emission component generated in the envelope (long-dashed line in Figure 201). The $R_{c}$ value of $100 \mathrm{AU}$ was chosen to reproduce the SED shape from about 15 to $50 \mu \mathrm{m}$. A larger $R_{c}$ would result in a flatter SED, while a smaller value would result in more mid-IR flux and a pronounced silicate absorption feature. Choosing $\eta_{\text {star }}=0.9$ and $\theta=$ $15^{\circ}$, combined with the high inclination angle, depresses the emission below $10 \mu \mathrm{m}$. Our model does not reproduce the deep ice features at 6.0 and $6.8 \mu \mathrm{m}$, but it traces the continuum level between 5 and $8 \mu \mathrm{m}$ (where emission from the disk dominates). We note that the IRS fluxes are lower than the IRAC data points; this is likely due to the extended emission detected in IRAC Luhman et al. 2006) and thus an aperture effect. Below $2 \mu \mathrm{m}$, the nearIR scattered light component from the envelope is likely overestimated. We note that even though the luminosity of our model, $4.0 \mathrm{~L}_{\odot}$, is larger than the measured bolometric luminosity of $2.5 \mathrm{~L}_{\odot}$ (see Table 4), it is necessary to yield enough flux at all wavelengths.

\subsubsection{5. $04365+2535$}

This IRAS source is also known as TMC 1A; it is optically invisible and thus deeply embedded. It is surrounded by a cometary nebulosity in the near-infrared (Tamura et al. 1991, 1996), and it is extended both in the near-IR and at $\mathrm{mm}$ wavelengths (Park \& Kenvon 2002). Both Chandler et al. (1996) and Tamura et al. (1996) measured a bipolar outflow which is somewhat conical in shape due to the blueshifted lobe being more prominent than the redshifted one. The near-IR nebulosity is likely light from the central source scattered by dust in the outflows (Tamura et al. 1996). Also, dust continuum measured at $790 \mu \mathrm{m}$ and $1.1 \mathrm{~mm}$ is aligned with the outflow direction (Chandler et al. 1998). Chandler et al. (1996) concluded that the inclination angle of this object lies between $40^{\circ}$ and $68^{\circ}$, with a cavity of semi-opening angle between $15^{\circ}$ and $21^{\circ}$.

The SED of $04365+2535$ is fitted by an envelope model with relatively high density $\left(\rho_{1}=4.5 \times 10^{-14} \mathrm{~g} \mathrm{~cm}^{-3}\right)$, a centrifugal radius of $50 \mathrm{AU}$, a small cavity, and an inclination angle of $30^{\circ}$ (Figure 21). Compared to models by $\mathrm{KCH} 93$, both our reference density and centrifugal radius are smaller, by factors of 0.45 and 0.17 , respectively, and our model suggests a more face-on orientation as opposed to the more highly inclined model $\left(i=60^{\circ}\right)$ of KCH93 and the inclination angle determined from the outflows (Chandler et al. 1996). More recent models by Whitney et al. (1997) and Gramajo et al. (2007) resulted in a large inclination angle $\left(70^{\circ}-80^{\circ}\right)$, but in values for $R_{c}$ and $\rho_{1}$ very comparable to ours.

The high density is required to fit the deep silicate absorption feature, as well as to shift the peak of the SED to longer wavelengths. Our model does not fit the sub$\mathrm{mm}$ and $\mathrm{mm}$ fluxes measured with a large aperture $\left(40^{\prime \prime}\right.$ $\left.60^{\prime \prime}\right)$, but matches the measurements done with apertures $\lesssim 20^{\prime \prime}$ (see Table 2). This suggest the presence of some 
extended, cold dust beyond the infalling envelope. A centrifugal radius of $50 \mathrm{AU}$ was chosen to fit the emission from the silicate absorption feature out to about $50 \mu \mathrm{m}$; a model with larger values for $\rho_{1}$ and $R_{c}\left(\sim 6.0 \times 10^{-14}\right.$ $\mathrm{g} \mathrm{cm}^{-3}$ and $70 \mathrm{AU}$, respectively) would also yield a good fit, but underestimate the emission in the $20-30 \mu \mathrm{m}$ range somewhat. An even larger centrifugal radius would decrease the emission from 15 to $40 \mu \mathrm{m}$. The inclination angle of our envelope model is smaller than the value suggested by Chandler et al. (1996) and by previous models; however, we can exclude a larger inclination angle, since it would result in a steeper SED from 15 to $60 \mu \mathrm{m}$, as well as less flux in the 2 to $10 \mu \mathrm{m}$ region. We can also exclude a cavity semi-opening angle larger than about $5^{\circ}$, since it would decrease the flux in the $5-15 \mu \mathrm{m}$ wavelength range.

\subsubsection{6. $04368+2557$}

This object has a bolometric temperature of less than $70 \mathrm{~K}$, thus placing it in the Class 0 domain, the earlier, deeply embedded protostellar stage (Chen et al. 1995; Motte \& André 2001). It is very faint and extended in the near-IR (Park \& Kenyon 2002); no point source is detected in $2 \mathrm{MASS}$. The $K$-band data point in our SED plot is from Whitney et al. (1997), who only detected some faint reflection nebulosities at the position of the IRAS source. Since the central source is not detected in the near-IR, Tamura et al. (1996) conclude that this object might be seen edge-on, obscured by a disk.

Our IRS observations were centered on the position determined by interferometric observations at $2.7 \mathrm{~mm}$ by Ohashi et al. (1997a), which also coincides with the position of the source in high-resolution $7 \mathrm{~mm}$ VLA maps by Loinard et al. (2002). The latter authors resolved this IRAS source into two components separated by 0 ". 17 and suggested that it is a binary in which one component is surrounded by an almost edge-on disk. In addition, this object might have a faint companion located $20^{\prime \prime}$ to the northwest of the main source, but it was only detected at $800 \mu \mathrm{m}$ (Fuller, Ladd, \& Hodapp 1996) and not confirmed at $1.3 \mathrm{~mm}$ (Motte \& André 2001) and 2.7 mm (Ohashi et al. 1997a).

IRAS $04368+2557$ drives molecular outflows, which are located symmetrically around the central source and also perpendicular to the elongated source detected by Loinard et al. (2002). Hogerheijde et al. (1998) inferred an inclination angle $i>65^{\circ}$ from observations of the outflows. Ohashi et al. (1997a) observed this object at high resolution in the millimeter wavelength range and concluded that it is surrounded by an elongated envelope $\sim 2000 \mathrm{AU}$ in radius with large bipolar cavities, seen edge-on.

The SED of IRAS $04368+2557$ (Figure 22) is similar to that of IRAS $04302+2247$ (Figure 17); both objects have a deep silicate absorption feature and SEDs that peak between 60 and $70 \mu \mathrm{m}$. Both are seen close to edgeon, but the reference density of the IRAS $04368+2557$ model fit is higher $\left(\rho_{1}=4.0 \times 10^{-14} \mathrm{~g} \mathrm{~cm}^{-3}\right)$, and the centrifugal radius smaller $\left(R_{c}=200 \mathrm{AU}\right)$. On the other hand, about $20 \%$ changes in $\rho_{1}$ and $R_{c}$ yield comparable model fits, as long as the cavity semi-opening angle lies between $20^{\circ}$ and $30^{\circ}$, and the source is seen edge-on. We can exclude a high density as derived by KCH93 (3.2 $\times 10^{-13} \mathrm{~g} \mathrm{~cm}^{-3}$ ), but agree reasonably well with their result of $R_{c}(300 \mathrm{AU})$ and inclination angle $\left(60^{\circ}-90^{\circ}\right)$.

In the near-IR, our model reproduces the $K$-band measurement, but seems to overestimate the 3.6 and $4.5 \mu \mathrm{m}$ emission, as measured by IRAC (Hartmann et al. 2005). However, this object is extended in IRAC images, and the photometry from the $6^{\prime \prime}$ aperture shown in Figure 22 does not capture all of the strong extended emission (see also Hartmann et al. 2005).

As with IRAS $04302+2247$, the values for $\rho_{1}$ and $R_{c}$ are fairly well constrained by the depth of the silicate absorption feature, the shape of the IRS spectrum beyond $10 \mu \mathrm{m}$, and the width and peak of the SED (the 100, 160 , and $1300 \mu \mathrm{m}$ measurements are likely overestimates due to aperture effects). The similarity between the two IRAS sources suggests that some Class I objects, seen at high inclination angles, could resemble Class 0 objects.

\subsubsection{7. $04381+2540$}

This IRAS source is also known as TMC 1; it is invisible in the optical and thus deeply embedded (e.g., Moriarty-Schieven et al. 1992). It is surrounded by a near-infrared nebulosity seen in scattered light (Tamura et al. 1991; Terebey et al. 2006). The conical shape of this near-infrared emission indicates that it likely traces an evacuated outflow cavity; the $\mathrm{CO}$ molecular outflow has a semi-opening angle between $13^{\circ}$ and $19^{\circ}$ and an inclination angle between $40^{\circ}$ and $70^{\circ}$ (Chandler et al. 1996).

Dust emission oriented perpendicular to outflows was detected at $790 \mu \mathrm{m}$ (Chandler et al. 1998). Young et al. (2003) measured extended 450 and $850 \mu \mathrm{m}$ emission from this object; based on the source's properties and model calculations, they confirmed that this object is a Class I source. From radial intensity profile fits to the $1.3 \mathrm{~mm}$ map, Motte \& André (2001) derived an outer envelope radius of 3900 AU. Recently, Apai et al. (2005) found a 0 .'6 companion to IRAS $04381+2540$; they suggest that this companion might be a young brown dwarf.

A good model fit for IRAS 04381+2540 (see Figure 23) requires a somewhat higher luminosity than its bolometric luminosity of $0.7 \mathrm{~L}_{\odot}$ (see Table 4) to be able to reproduce the long-wavelength emission. By increasing the inner disk radius to 5 stellar radii, the emission in the near-IR and mid-IR out to $8 \mu \mathrm{m}$ is decreased, resulting in a better fit. The centrifugal radius of $70 \mathrm{AU}$ and the reference density of $3.0 \times 10^{-14} \mathrm{~g} \mathrm{~cm}^{-3}$ are well constrained by the depth of the silicate absorption feature and the shape of the IRS spectrum from 15 to $30 \mu \mathrm{m}$. Decreasing $R_{c}$ to $60 \mathrm{AU}$ and the inclination angle to $35^{\circ}$ yields a nearly identical fit, but we can exclude larger changes in these parameters, as well for the reference density.

Previous modeling by KCH93 yielded a value of $1.0 \times$ $10^{-13} \mathrm{~g} \mathrm{~cm}^{-3}$ for $\rho_{1}$ and $300 \mathrm{AU}$ for $R_{c}$, and an inclination angle of $30^{\circ}$. However, better-constrained models by Whitney et al. (1997) resulted in values for $\rho_{1}$ and $R_{c}$ similar to ours. Eisner et al. (2005) derived a smaller centrifugal radius (30 $\mathrm{AU})$ and also a larger reference density $\left(6.75 \times 10^{-14} \mathrm{~g} \mathrm{~cm}^{-3}\right)$.

Our results for cavity semi-opening angle $\left(\theta=10^{\circ}\right)$ and inclination $\left(i=40^{\circ}\right)$ are at the lower limits of the range suggested by Chandler et al. (1996). Our value for $\theta$ agrees well with the modeling result of Whitney et al. (1997), but our inclination angle is smaller; on the other hand, this latter quantity is consistent with the result 
of Terebey et al. (2006), but not with their cavity semiopening angle of $40^{\circ}$. Higher values for both parameters can be excluded based on the constraints placed by our IRS spectrum on the envelope models. Finally, we note that we adopted an outer envelope radius of $10000 \mathrm{AU}$, which is larger than the value derived by Motte \& André (2001), but necessary to fit the long-wavelength part of the SED.

\subsubsection{8. $04489+3042$}

Based on the M6 spectral type of IRAS $04489+3042$, White \& Hillenbrand (2004) indicated that it could be substellar, a Class I brown dwarf. However, recent spectral type determinations by Luhman (2006) suggest that its spectral type is M3-M4, thus placing it into the low-mass star regime. IRAS $04489+3042$ is not extended in the near-IR or mm (Park \& Kenyon 2002; Motte \& André 2001), and no outflow has been detected (Gomez et al. 1997). Park \& Kenyon (2002) suggest it could be in transition between Class I and II stage, probably seen close to pole-on.

Our preliminary envelope model fit for this object (Figure 24) suggests a low density $\left(\rho_{1}=1.0 \times 10^{-14} \mathrm{~g} \mathrm{~cm}^{-3}\right)$, a small centrifugal radius $\left(R_{c}=15 \mathrm{AU}\right)$, a very small cavity, and a low inclination. Models by KCH93 yielded the same reference density as our model and a similar low inclination angle, but a centrifugal radius of $70 \mathrm{AU}$. On the other hand, modeling efforts by Whitney et al. (1997) resulted in larger values for all these three parameters. We can exclude a centrifugal radius larger than about $25 \mathrm{AU}$, since it would decrease the flux over the $10-60 \mu \mathrm{m}$ spectral range. The low density and inclination angle of our model are mainly set by the very weak silicate absorption feature and roughly flat SED from about 5 to 15 $\mu \mathrm{m}$. The model does not yield a good fit of the details of the IRS spectrum below about $15 \mu \mathrm{m}$, but the overall shape of the spectrum is reproduced reasonably well. To fit the data point at $1.3 \mathrm{~mm}$ with the set of model parameters described above, we adopted an outer envelope radius of only $1000 \mathrm{AU}$; the small envelope contributes only little emission in the $\mathrm{mm}$, where the emission from the disk dominates (see the dash-dotted line in Figure 24). Since there are no sub-mm measurements available, and the IRS spectrum is relatively flat with no strong silicate feature, the current model parameters are only weakly constrained.

\subsubsection{9. СoKu Tau/1}

Coku Tau/1 was identified as a Class II object by KH95, but it is surrounded by filamentary reflection nebulae detected in the near-IR and suggested to represent the walls of outflow cavities (Padgett et al. 1999). It is seen close to edge-on, and, like IRAS $04248+2612$, is a subarcsecond binary (Padgett et al. 1999). Similar to IRAS $04248+2612$ and $04264+2433$, CoKu Tau/ 1 has a silicate emission feature and an infrared SED that is rising from 3 to about $20 \mu \mathrm{m}$ (see Figure 3).

The appearance of CoKu Tau/1 in the near-IR suggests that it is surrounded by a low-density envelope and an edge-on disk. It is likely a more evolved protostar, at a stage when the envelope has begun dissipating. The binary and the inner disk are extinguished by the outer disk, but since in the near-IR the optical depth of the outer regions of the circumbinary disk decreases, the two stars at the center are seen at near-IR wavelengths. As in the optical, there is likely also some stellar light scattered by the envelope contributing to the near-IR emission.

Our model of CoKu Tau/1 (see Figure 25) requires a low density $\left(\rho_{1}=5.0 \times 10^{-15} \mathrm{~g} \mathrm{~cm}^{-3}\right)$, a small centrifugal radius $\left(R_{c}=40 \mathrm{AU}\right)$, a cavity semi-opening angle of $5^{\circ}$, and a high inclination angle $\left(i=80^{\circ}\right)$. We adopted a luminosity of $1.1 \mathrm{~L}_{\odot}$, which is slightly higher than the measured bolometric luminosity of $1.0 \mathrm{~L}_{\odot}$ (see Table 4). The inner disk radius was set at 7 stellar radii to decrease the emission from the near-IR to $8 \mu \mathrm{m}$, which is still higher than the observed fluxes, most likely due to an overestimate of the scattered light emission from the envelope.

The shape of the IRS spectrum between 12 and $20 \mu \mathrm{m}$ constrains the centrifugal radius, while the low density is required to generate a silicate emission feature and cause the SED to peak around $30 \mu \mathrm{m}$. Decreasing $\rho_{1}$ by about $20 \%$ and increasing or decreasing $R_{c}$ by roughly the same percentage yields comparable fits, provided the inclination angle lies in the $70^{\circ}-80^{\circ}$ range. A previous model by Stark et al. (2006) yielded a reference density smaller by about a factor of three, a comparable values for $R_{c}$, but a somewhat smaller inclination angle and a larger cavity opening angle. We can exclude a larger cavity, since it would decrease the flux of the $10 \mu \mathrm{m}$ silicate emission feature.

As mentioned earlier, the silicate emission feature at $10 \mu \mathrm{m}$ is generated by the envelope. The disk just contributes a weak silicate absorption feature, which results from our simple disk model that includes only continuum emission, extinguished by the envelope. A more realistic edge-on disk would yield a somewhat stronger absorption feature; however, the envelope emission clearly dominates over that of the disk at $10 \mu \mathrm{m}$, a fact that also applies to IRAS $04264+2433$ and, to a lesser extent, IRAS 04248+2612 (long-dashed gray lines in Figures 14. 15. 25). Thus, the silicate emission feature is not greatly affected by the shape of the silicate feature originating from the disk, and our current models of low-density envelopes have some validity.

\subsubsection{DG Tau B}

This object displays some similarity to $\mathrm{HH} 30$; its nearIR HST images show an edge-on disk and a bipolar reflection nebulosity (see Fig. 1), which was interpreted as the walls of an outflow cavity (Padgett et al. 1999). In the optical, the nebula is visible, too, but the star is not detected (Stapelfeldt et al. 1997). DG Tau B is also the source of a jet, which is oriented perpendicular to the disk and along the axis of symmetry of the reflection nebula (Eislöffel \& Mundt 1998). A molecular outflow, oriented along the redshifted optical jet, has also been observed (Mitchell, Sargent, \& Mannings 1997).

Our preliminary model for DG Tau B (see Figure 26) has an inclination angle of $55^{\circ}$ and a cavity semi-opening angle of $10^{\circ}$; both values are smaller than expected from the optical and near-IR images. However, a more highly inclined envelope would result in a steeply rising SED over the mid-IR range, which would not match the observed spectrum. The centrifugal radius (and thus outer disk radius) of $60 \mathrm{AU}$ is poorly constrained; a larger value would better fit the slope of the IRS spectrum beyond 
$20 \mu \mathrm{m}$, but result in a shallower silicate feature at 10 $\mu \mathrm{m}$. The deep silicate absorption feature requires a relatively high density $\left(\rho_{1}=3.5 \times 10^{-14} \mathrm{~g} \mathrm{~cm}^{-3}\right)$, but its long-wavelength wing is not fitted very well. In addition, the model is not well-constrained at longer wavelengths, since DG Tau B lacks any sub-mm measurements, and the IRAS fluxes at 60 and $100 \mu \mathrm{m}$ are uncertain due to the proximity of DG Tau: the far-IR fluxes contain the emission of both DG Tau B and DG Tau, which lies 1' to the northeast of DG Tau B and is about two to three times brighter in the mid-IR.

Recent models by Stark et al. (2006) yielded quite different model parameters, except for the reference density; they were able to fit the near-IR images of DG Tau $\mathrm{B}$ adopting a luminosity of $0.2 \mathrm{~L}_{\odot}$, a centrifugal radius of $300 \mathrm{AU}$, a $\rho_{1}$ value of $3.75 \times 10^{-14} \mathrm{~g} \mathrm{~cm}^{-3}$, and cavity semi-opening and inclination angles of $30^{\circ}$ and $73^{\circ}$, respectively. This set of parameters would not fit the IRS spectrum. In particular, we adopted a luminosity of $2.5 \mathrm{~L}_{\odot}$; a smaller luminosity, closer to the value derived from integrating under the SED $\left(1.8 \mathrm{~L}_{\odot}\right)$, would result in less far-IR flux and require a smaller inclination angle to still reproduce the mid-infrared spectrum, which would be inconsistent with the HST images, which indicate a more edge-on orientation.

\subsubsection{HL Tau}

Even though HL Tau is considered by some a classical $\mathrm{T}$ Tauri star surrounded by an accretion disk, it is also embedded in an envelope (e.g., Motte \& André 2001; White \& Hillenbrand 2004); this is supported by our IRS spectrum, which shows the silicate feature in absorption, as well as ice absorption features in the 5-8 $\mu \mathrm{m}$ range and at $15.2 \mu \mathrm{m}$. HL Tau is also very bright at sub-mm and $\mathrm{mm}$ wavelengths, and a rotating, elongated, $2000 \mathrm{AU}$ long structure has been mapped in ${ }^{13}$ CO (Beckwith et al. 1990; Sargent \& Beckwith 1991). Stapelfeldt et al. (1995) detected a compact, one-sided reflection nebulosity in the optical; the star is not detected. HL Tau drives a powerful, extended molecular outflow which was mapped in CO emission (Monin et al. 1996). Close et al. (1997) observed HL Tau in the nearIR with adaptive optics and resolved the inner accretion disk and bipolar outflow cavities. They concluded that HL Tau is surrounded by an accretion disk of about 150 $\mathrm{AU}$ radius, inclined at $67^{\circ}$, and an infalling envelope of about 1200 AU radius. However, the environment of HL Tau is quite complicated, partly due to its proximity to XZ Tau, which is likely at the origin of an expanding shell that compresses the nebula around HL Tau (Welch et al. 2000).

The SED of HL Tau can be reproduced with an envelope model with a luminosity of $8.0 \mathrm{~L}_{\odot}$, which is somewhat larger than its measured bolometric luminosity (see Table 4), a relatively high density $\left(\rho_{1}=4.5 \times 10^{-14} \mathrm{~g}\right.$ $\mathrm{cm}^{-3}$ ), an $R_{c}$ value of $100 \mathrm{AU}$, and flattening parameter $\eta$ of 1.0, i.e., a sheet-collapse model (Figure [27). The latter quantity was adjusted to yield more flux in the 12 to $30 \mu \mathrm{m}$ region compared to the TSC models. A lower density would yield a $10 \mu \mathrm{m}$ silicate feature that is too narrow, while a density up to $6.0 \times 10^{-14} \mathrm{~g} \mathrm{~cm}^{-3}$ would still yield a good fit. An even higher density would require a larger centrifugal radius, which would result in an SED that overestimates the flux from about 25 to 200 $\mu \mathrm{m}$. The $R_{c}$ value is constrained by the shape of the IRS spectrum; we can rule out $R_{c}$ values smaller than $100 \mathrm{AU}$ and larger than about $200 \mathrm{AU}$. These results are also roughly consistent with previous modeling results by Calvet et al. (1994), who adopted a reference density of $3.2 \times 10^{-14} \mathrm{~g} \mathrm{~cm}^{-3}$ and a centrifugal radius of $200 \mathrm{AU}$.

We note that, despite the finding by Close et al. (1997) that the inclination angle of HL Tau is $67^{\circ}$ and that large cavities are present, our model indicates virtually no cavity and a low inclination. The model by Calvet et al. (1994) also suggests an outflow hole with a semi-opening angle of $10^{\circ}$; including such a cavity in our model would reduce the flux over the IRS wavelength range, but also increase the flux somewhat in the near-IR, where our model underestimates the emission. A larger inclination angle would decrease the short-wavelength flux even more and generate a deeper silicate absorption feature. On the other hand, our value for the inclination angle is closer to the value of $30^{\circ}$ determined by Calvet et al. (1994), and our disk radius $\left(\sim R_{c}\right)$ is also roughly consistent with the value of $150 \mathrm{AU}$ found by Close et al. (1997).

Our model underestimates the emission at sub$\mathrm{mm}$ and $\mathrm{mm}$ wavelengths, despite an outer envelope radius of $10,000 \mathrm{AU}$. Models of $\mathrm{HL}$ Tau by D'Alessio, Calvet, \& Hartmann (1997) have shown that the outer disk requires higher temperatures to reproduce the SED shape at longer wavelengths, implying irradiation of the circumstellar disk by the infalling envelope. Since our model does not include this additional heating, our disk emission is likely underestimated; thus, the "missing" flux at the longer wavelengths could be attributed in part to this additional disk component. In addition, dust outside the infall region could contribute to the mm emission.

\subsubsection{L1551 IRS 5}

This object is the most luminous Class I object in Taurus $\left(\mathrm{L}_{b o l}=22-28 \mathrm{~L}_{\odot}\right.$; see Table 4 ) and is therefore a wellstudied protostellar system. It consists of a $0 . .3$ binary, whose components are each surrounded by a circumstellar disk, and of a circumbinary disk and a flattened envelope (Looney, Mundy, \& Welch 1997; Rodríguez et al. 1998). It is surrounded by an extended reflection nebula at optical and near-IR wavelengths (Campbell et al. 1988; Tamura et al. 1991; White et al. 2000), and it powers a highly collimated bipolar molecular outflow (Snell \& Schloerb 1985; Moriarty-Schieven et al. 1992). Even though no optical outburst has been observed, L1551 IRS 5 is considered an FU Ori object, implying episodic events of high disk accretion and outflow rates (Hartmann \& Kenyon 1996).

The mid- and far-IR spectrum $(2-200 \mu \mathrm{m})$ of this bright Class I object was obtained by White et al. (2000) using ISO; they detected gas-phase emission lines and ice absorption features. We also detect strong ice absorption features in our IRS spectrum, in addition to a very deep and broad silicate absorption feature (see Figure 2k).

L1551 IRS 5 has been modeled in detail by Osorio et al. (2003), taking into account emission from the circumstellar and circumbinary disks, as well as the envelope. The model presented below does not include all the components of the system as in Osorio et al. (2003) 
and should therefore be considered as preliminary.

L1551 IRS 5 is the most luminous object in our sample (adopted luminosity of $25 \mathrm{~L}_{\odot}$ ). Its deep silicate absorption feature requires a high density $\left(\rho_{1}=7.0 \times 10^{-14}\right.$ $\mathrm{g} \mathrm{cm}^{-3}$ ), and the steep SED beyond $15 \mu \mathrm{m}$ a centrifugal radius of $100 \mathrm{AU}$ (see Figure 28). An $R_{c}$ value larger than about $150 \mathrm{AU}$ would result in a decrease in flux between 15 and $30 \mu \mathrm{m}$ and thus degrade the fit to the IRS spectrum. Our model indicates that the cavity is small $\left(\theta=5^{\circ}\right)$, and the object is seen at an intermediate inclination angle. Increasing $\rho_{1}$ would require a smaller inclination angle, but a larger inclination is more in accordance with the observed outflows $\left(i=65^{\circ}\right.$; Hogerheiide et al. 1998).

Compared to the models by KCH93, which suggested a reference density of $\sim 10^{-13} \mathrm{~g} \mathrm{~cm}^{-3}$, a centrifugal radius between 70 and $300 \mathrm{AU}$, and an inclination angle between $30^{\circ}$ and $60^{\circ}$, our model has a smaller, but still high, reference density, an $R_{c}$ value towards the lower end of the KCH93 range, and an inclination angle in the middle of the range determined by KCH93. Whitney et al. (1997) and, more recently, Gramajo et al. (2007), modeled this object with a reference density of only $3.75 \times 10^{-14} \mathrm{~g}$ $\mathrm{cm}^{-3}, R_{c} \sim 40 \mathrm{AU}, \theta=20^{\circ}$, and $i \gtrsim 70^{\circ}$.

Our model parameters are different from the ones found by Osorio et al. (2003), who adopted an inclination angle of $50^{\circ}, \rho_{1}=4.0 \times 10^{-13} \mathrm{~g} \mathrm{~cm}^{-3}, R_{c}=300$ $\mathrm{AU}$, and a flattening parameter $\eta=2.5$ for the envelope. We could obtain a comparable fit of the SED out to $30 \mu \mathrm{m}$ by using $L=25 \mathrm{~L}_{\odot}, \rho_{1}=2.5 \times 10^{-13} \mathrm{~g} \mathrm{~cm}^{-3}$, $R_{c}=100 \mathrm{AU}, \eta=2.0, \eta_{\text {star }}=0.1, \theta=0.1^{\circ}$, and $i=45^{\circ}$, but the far-IR emission would be overestimated. On the other hand, the sub-mm and mm emission would be better matched with the sheet-collapse model. Thus, the SED of L1551 IRS 5 can be reproduced by a TSC model, but the long-wavelength emission suggests the presence of an additional, cold, likely elongated, dust component that is outside of the infall region.

\section{DISCUSSION}

\subsection{Identification of Class I Objects}

The mid-infrared spectra of Class I objects have quite different appearances; while some differences are caused by individual envelope parameters like the density and size of the infall region, the inclination angle also plays an important role. A Class I object viewed pole-on through a cavity in the envelope can look like a Class II object with some additional long-wavelength excess emission (as some FU Ori objects appear; see Green et al. 2006), while an edge-on Class I object can have the appearance of a Class 0 object. However, the envelope around a true protostar will generate excess emission at mid-IR to $\mathrm{mm}$ wavelengths, resulting in a larger infrared excess than expected from an accretion disk alone, and less excess when compared to the large, cool envelopes around Class 0 objects that peak at sub-mm to mm wavelengths. In addition, if seen within a narrow range of inclination angles, an edge-on Class II object can appear similar to an embedded protostar (see D'Alessio et al. 1999), but it would lack additional excess emission caused by the envelope.

Observations at other wavelengths help in identifying the evolutionary state of an object: as noted by
Park \& Kenyon (2002), true Class I sources are usually extended in the near-IR and at sub-mm or $\mathrm{mm}$ wavelengths, the former due to light scattered by dust in inner envelope regions, the latter due to thermal emission by dust in the outer parts of the envelope. Furthermore, Class I objects usually have molecular outflows or jets detected in the optical/near-IR and especially at $\mathrm{mm}$ wavelengths. Objects in transition between the Class I and Class II stage are expected to be surrounded by less envelope material, in accordance with larger cavities in their envelopes.

Of the 28 Class I objects presented in this paper, 7 were previously identified as Class II objects in KH95: IRAS 04154+2823, 04158+2805, 04278+2253, CoKu Tau/1, HL Tau, IC 2087 IR, and LkHa 358. While the SED shape of these objects might indicate that they are $\mathrm{T}$ Tauri stars whose envelope has already mostly dissipated, the presence of ice features in their mid-infrared spectrum, as well as the detection of extended reflection nebulosities and outflows around some of these objects, suggests that there is still some remnant envelope material.

IRAS 04154+2823, 04278+2253, 04489+3042, IC 2087 IR, and LkHa 358, as well as some of the Class II objects presented in Furlan et al. (2006) (IRAS 04187+1927, DG Tau, DP Tau, FS Tau, HN Tau, T Tau, and XZ Tau), could be objects in transition between the Class I and II stage, when a protostar has almost completely cleared its envelope, but is still surrounded by some nebulosity. In some cases, the "evolved" protostar is still generating outflows, but due to envelope clearing it appears more and more like a classical $\mathrm{T}$ Tauri star. In some other cases, like T Tau, HN Tau, and XZ Tau, a binary consists of a T Tauri star and a more deeply embedded source, which is likely an effect of the particular viewing geometry of the system: either one component is more aligned with the outflow cavity and thus less embedded, or the more extinguished component is actually a $\mathrm{T}$ Tauri star oriented edge-on. Therefore, as stated earlier, the inclination angle is an important factor in determining the appearance of Class I and II objects.

\subsection{Ices in the Envelopes of Class I Objects}

The ice and silicate absorption features detected in the IRS spectra are also a useful tool to characterize Class I objects. The peak optical depth of these features reveals their strength and can therefore be used as an indication for their origin. To determine the optical depth of the $\mathrm{CO}_{2}$ ice feature at $15.2 \mu \mathrm{m}$ and that of the silicate absorption feature around $10 \mu \mathrm{m}$, we first used a spline fit to determine the underlying continuum. Then we derived the optical depth, $\tau$, assuming $F_{o b s}=F_{c} e^{-\tau}$, where $F_{o b s}$ and $F_{c}$ are the observed and the underlying continuum flux, respectively. The measured peak optical depths of the $\mathrm{CO}_{2}$ and silicate absorption features are shown in Figure 29, together with previous measurements by Gibb et al. (2004) and Alexander et al. (2003), who determined the optical depths of silicate and ice absorption features in ISO spectra of embedded, massive YSOs and low- and intermediate-mass YSOs, respectively. For the Alexander et al. (2003) data, we used the same conversion as in Watson et al. (2004) to convert the $\mathrm{CO}_{2}$ equivalent widths to peak optical depths.

As already pointed out by Watson et al. (2004), the low-mass Class I objects in Taurus generally have large 
peak $\mathrm{CO}_{2}$ optical depths, especially considering that their peak silicate optical depth lies below $\sim 2$ (the object with the largest silicate and $\mathrm{CO}_{2}$ optical depths is IRAS $04368+2557$, a Class 0 object). Most of the objects observed by Alexander et al. (2003) have similarly strong $\mathrm{CO}_{2}$ ice features for larger silicate optical depths, suggesting additional extinction along the line of sight towards these objects. It is expected that the two optical depths track each other along lines of sight through molecular clouds (with some dispersion due to different conditions in different clouds), since ice mantles are thought to grow in a similar manner onto dust grains in all comparably shielded regions of molecular clouds.

The gray dashed line in Figure 29 is drawn by eye to roughly separate the region occupied by the Taurus Class I objects, where extinction from the envelope dominates the optical depth, from that occupied by objects seen along molecular cloud lines of sight, where the extinction is mostly due to intervening molecular cloud material. The boundary is not well-defined, since some of the ISO observations did include objects in which local extinction by an envelope dominates, but it separates all but one of the Taurus Class I objects from the majority of the ISO targets.

Thus, we confirm the conclusions of Watson et al. (2004) that dust grains in protostellar envelopes have larger ice mantles than grains in the ambient molecular cloud, implying ice mantle growth inside the envelopes. We defer a more detailed study of the ice absorption features, including the detailed composition and thus derived temperature of the ices, to a future paper (Zasowski et al. 2008, in preparation).

We note that the objects with a peak silicate optical depth of 0 are protostars in which the silicate feature is either in emission or displays both an emission and an absorption component. As discussed in $\S 3.1$ the latter type of objects is likely seen along lines of sight that suffer from less extinction by the envelope, caused by a more face-on orientation or a lower-density envelope. Therefore, for these objects the optical depth of the silicate feature, taken as a measure of dust absorption in the envelope, is probably underestimated due to contamination by the silicate emission arising from the disk.

The one Class I object that lies in the molecular cloud region of the plot is $\mathrm{DG} \mathrm{Tau} / \mathrm{B}$; it has a deep silicate, but only a weak $\mathrm{CO}_{2}$ ice absorption feature. This could indicate that grain mantles in its envelope are less rich in $\mathrm{CO}_{2}$ ice, but on the other hand this feature is superposed on the broad and deep silicate absorption at $18 \mu \mathrm{m}$, which adds additional uncertainty to the optical depth measurement of the $\mathrm{CO}_{2}$ feature.

The three objects identified as "Class I/II objects" in Figure 29 are IRAS 04278+2253, LkHa 358, and IC 2087 IR. The former two objects have the lowest $\mathrm{CO}_{2}$ feature strengths of our Taurus sample and virtually no silicate absorption feature, which confirms that they are more evolved Class I objects. IC 2087 IR has both $\mathrm{CO}_{2}$ and silicate peak optical depths comparable to those of typical Class I objects; thus, it is still likely embedded to some extent. This shows that the optical depths of the silicate and ice absorption features can help to recognize more evolved Class I objects, but the effects of the environment and viewing angle also have to be taken into account.

\subsection{Summary of the Models}

Envelope models aid in the identification and characterization of Class I objects; when models fit the SED from the near-IR to the $\mathrm{mm}$, they reveal both the largeand small-scale structure of a protostar, its accretion disk and its envelope. The IRS spectrum, which covers the SED from 5 to $40 \mu \mathrm{m}$, is a particularly useful constraint, since the depth of the silicate feature at $10 \mu \mathrm{m}$ is connected to the density and inclination angle of the envelope, while the slope between 15 and $40 \mu \mathrm{m}$ helps to constrain the envelope shape, centrifugal radius and in part the size of the cavity. Not all parameters can be uniquely defined by a model fit to the IRS spectrum, but together with shorter- and longer-wavelength data, the parameter space can be narrowed down considerably.

We point out that, given the large number of parameters for each model, we did not determine the goodness of a model fit by $\chi^{2}$-minimization, but rather by judging by eye, based on our experience on how the different parameters affect the resulting model SED. However, we justify the choice of specific parameters and explain how the parameter space was narrowed. In addition, the more constraints a model has, in particular the availability of sub-mm and mm flux measurements and the source's luminosity, the more limited the choice of parameters becomes. The fitting by eye is much more efficient than $\chi^{2}$-minimization, and in most cases we derive good fits that are likely minima in $\chi^{2}$ space. Typical uncertainties for our model parameters are listed in the footnote of Table 3 .

We also note that several assumptions made in the modeling code could be refined to better reproduce the observations: e.g., the assumption that the cavity follows the shape of the streamlines of infalling particles, the assumption of a spherically symmetric density distribution when computing the equilibrium temperature in the envelope, and the adoption of only the inner, optically thick regions of a flat accretion disk as the disk component. Some of our 22 models of Class I objects should be treated as preliminary, given the assumptions made in the modeling code and/or some weaker constraints due to the lack of data, but our models already reveal a large range in parameters necessary to obtain fits (see Table 3 for a summary of the model parameters).

We computed models for the majority of Class I objects in our sample; however, we did not attempt to model objects with poor observational constraints for their SEDs, or objects which are likely more evolved Class I objects and for which these envelope models might not apply. Thus, we did not attempt to model HH 30, whose spectrum is very noisy and whose overall SED is poorly constrained by the scarcity of measurements at other wavelengths. Also IRAS 04181+2654 B, a member of a binary system, was not modeled, since it lacks long-wavelength data (we modeled component $\mathrm{A}$, which was observed at $1.3 \mathrm{~mm})$. The three sources with decreasing SEDs over the IRS spectral range, IRAS $04278+2253$, IC 2087 IR, and LkHa 358, were also not modeled. Finally, no model was produced for GV Tau, since its IRS spectrum is uncertain, and the fact that the two components are separated by $1^{\prime \prime} 3$ (which corresponds to about $180 \mathrm{AU}$ at the distance of the Taurus star-forming region) likely introduces asymmetries in the envelope of the more embedded 
component.

Our envelope models indicate centrifugal radii, and thus disk sizes, that cover a range from 10 AU up to 300 AU, with a median value of 60 AU. Since the centrifugal radius is thought to increase rapidly with time $\left(\propto t^{3}\right)$, we could conclude that the objects in our sample span somewhat different ages (about a factor of 3 ). However, the main reason for the differences in centrifugal radii is likely different initial conditions of the collapse, since the initial centrifugal radius strongly depends on the mass of the central condensation and the initial angular velocity of the cloud core $\left(R_{c} \propto M^{3} \Omega^{2}\right.$; Terebey, Shu, \& Cassen 1984). In addition, some of the more deeply embedded protostars that have been identified as Class 0/I objects, like IRAS $04166+2706$ and $04368+2557$, have large $R_{c}$ values, which would not be expected if the spread in centrifugal radii was only due to time evolution. Since a disk size of a few $100 \mathrm{AU}$ is comparable to the radii of accretion disks found around classical T Tauri stars (e.g., Dutrey et al. 1996), our median value for $R_{c}$ (and thus outer disk radii) of 60 AU suggests that the accretion disks found around protostars will expand, as is expected due to angular momentum transfer in the accretion process.

We note that the disk component dominates the flux in the $3-8 \mu \mathrm{m}$ range for most of the models; for about $2 / 3$ of these objects the fraction of the luminosity contributed by the star, $\eta_{\text {star }}$, is either 0.1 or 0.2 , i.e., $80-90 \%$ of the luminosity is generated by accretion. About half of the objects that have no dominant disk contribution in the $3-8 \mu \mathrm{m}$ range have luminosities dominated by the star $\left(\eta_{\text {star }}=0.8\right)$, and the other half have smaller values for $\eta_{\text {star }}$, but generally large inclination angles. Thus, the Class I objects in our sample whose luminosity is dominated by accretion (and which likely have large mass accretion rates through their disks) generate SEDs that are dominated by the disk in the $3-8 \mu \mathrm{m}$ range, except if the disk emission is diminished due to a high inclination angle.

The reference density $\rho_{1}$ varies from $4.0 \times 10^{-15} \mathrm{~g} \mathrm{~cm}^{-3}$ to $7.0 \times 10^{-14} \mathrm{~g} \mathrm{~cm}^{-3}$, with a median value of $3.0 \times 10^{-14}$ $\mathrm{g} \mathrm{cm}^{-3}$, very similar to the value found by KCH93. $\rho_{1}$ can be used to estimate the mass infall rate from the envelope onto the disk by applying Equation[1 for a $0.5 \mathrm{M}_{\odot}$ object, the mass infall rates for the objects in our Class I sample lie between $5.3 \times 10^{-7}$ and $9.3 \times 10^{-6} \mathrm{M}_{\odot} \mathrm{yr}^{-1}$, about two orders of magnitude larger than the accretion rates of classical T Tauri stars (e.g., Gullbring et al. 1998). This would confirm previous results that Class I objects are at an earlier evolutionary stage than $\mathrm{T}$ Tauri stars and still accreting a substantial fraction of the final stellar mass (Muzerolle, Hartmann, \& Calvet 1998).

Even though the cavity semi-opening angle and, in some cases, the inclination angle are usually less wellconstrained than the reference density and centrifugal radius, we observe that our models indicate a large range of inclination angles, covering values from $15^{\circ}$ to $89^{\circ}$ (with a median value of $55^{\circ}$ ), and a smaller range of cavity semi-opening angles $\left(0.1^{\circ}\right.$ to $27^{\circ}$, with a median of $\left.6^{\circ}\right)$.

In Table 4 we list the luminosities of the Class I objects in our sample and their inclination angles. Both the values of bolometric luminosity from the literature and those we derived by integrating under the SED are actually apparent luminosities, since envelopes are not spherically symmetric. Thus, we would expect the apparent bolometric luminosity to be larger than the system luminosity (which enters as a model parameter) at low inclination angles, while at high inclination angles the opposite should be the case. The data in Table 4 shows that this trend is only weak for our sample of Class I objects; in general, our model luminosities are higher than the apparent bolometric luminosities. This could suggest that we overestimated the luminosity of some of our lower-inclination sources.

We note that most of our models do not require an initially flattened density distribution $(\eta \gtrsim 1)$; all but 5 of the 22 objects we modeled can be fitted by TSC models, which assume an initially spherically symmetric density distribution. However, we cannot exclude that sheetcollapse model fits could also be found for the remaining 17 Class I objects for which only TSC models have been considered so far. Thus, as a starting point for models, it seems that star-forming cores in Taurus can be described by the approximation of collapsing, singular isothermal spheres.

In a few cases we found that an additional component of cold dust seems to be present, since certain models are able to reproduce the sub-mm and $\mathrm{mm}$ fluxes measured in smaller apertures $\left(10^{\prime \prime}-20^{\prime \prime}\right)$, but not the measurements at similar wavelengths derived from large apertures $\left(40^{\prime \prime}-60^{\prime \prime}\right)$. In particular the $1.3 \mathrm{~mm}$ data points from Motte \& André (2001), which correspond to fluxes integrated over a beam $60^{\prime \prime}$ in diameter, are often higher than the model prediction. As suggested by Jayawardhana et al. (2001), cold dust of roughly constant density outside of the infall region could contribute to the long-wavelength emission. In these cases envelope models could aid in the distinction between emission from infalling envelope material and emission from the surrounding dense cloud.

When comparing our modeling results with previous efforts, we note that our results often differ considerably. However, in this work we have the additional constraints through our IRS spectra, which, when combined with near-IR and far-IR to $\mathrm{mm}$ data, allows us to determine the 9 parameters entering in the envelope models. In particular the $10 \mu \mathrm{m}$ silicate absorption feature allows us to narrow down the parameter space. A next step in modeling would involve reproducing the images of the Class I sources at the same time as the SEDs to better take into account the envelope geometry and asymmetries.

\section{CONCLUSIONS}

After analyzing the IRS spectra and SEDs of 28 Class I objects in the Taurus star-forming region, and generating envelope models for 22 of these objects, we conclude the following:

- Almost all protostars we observed display ice absorption features in their mid-IR spectra, which originate in the envelopes around these young stars. In particular, the $\mathrm{CO}_{2}$ ice feature at $15.2 \mu \mathrm{m}$ is ubiquitous and strong among the Class I objects in our sample, signifying an origin in the cold outer envelope regions. The envelope is also generally responsible for a deep silicate absorption feature at $10 \mu \mathrm{m}$. The three objects with silicate emission features in their spectra, IRAS 04248+2612, 04264+2433, and CoKu Tau/1 are likely Class I objects seen through low-density envelopes, which are in an ad- 
vanced stage of dispersal and thus considered to be more evolved.

- The SEDs of Class I objects peak in the mid- to far-IR, where the emission is dominated by the envelope. Objects with flat SEDs in the infrared spectral range could be in transition between the Class I and II stage. The inclination angle of protostellar systems strongly affects the appearance of their mid-IR SED; Class I objects seen pole-on could appear as Class II objects with additional long-wavelength excess emission, while edgeon Class I objects have SED shapes similar to Class 0 objects, but with less flux in the sub-mm and mm wavelength region.

- The IRS spectra are instrumental in constraining envelope model parameters, in particular the reference density $\rho_{1}$ (which is linked to the mass infall rate) and centrifugal radius $R_{c}$. The depth of the silicate feature and the shape of the spectrum between 12 and $30 \mu \mathrm{m}$ provide constraints which can be further tightened by model fits to near-IR, far-IR, sub-mm, and $\mathrm{mm}$ observations. The median values for $\rho_{1}$ and $R_{c}$ for the Class I objects we modeled are $3 \times 10^{-14} \mathrm{~g} \mathrm{~cm}^{-3}$ and $60 \mathrm{AU}$, respectively, with a relatively large range for both parameters. The disk contribution dominates the emission in the $3-8 \mu \mathrm{m}$ range for most of the modeled Class I objects, confirming the importance of disk accretion luminosity at this early evolutionary stage. Most SEDs can be reproduced by TSC models, thus not requiring an initially flattened density distribution of the collapsing cloud core; only 5 of the 22 objects we modeled needed sheet-collapse models for an adequate fit of their SEDs.

The IRS spectra of Class I objects in Taurus display a variety in appearances and add valuable constraints to envelope models. Fits to the observed SEDs yield the parameters describing their physical structure, and thus aid in confirming and refining our understanding of protostellar cloud collapse and evolution.

Part of this work was carried out while the first author was supported by a NASA Postdoctoral Program Fellowship at the NASA Astrobiology Institute at the University of California, Los Angeles, administered by Oak Ridge Associated Universities through a contract with NASA. This work is based on observations made with the Spitzer Space Telescope, which is operated by the Jet Propulsion Laboratory, California Institute of Technology, under NASA contract 1407. Support for this work was provided by NASA through contract number 1257184 issued by JPL/Caltech. N.C. and L.H. acknowledge support from NASA Origins grants NAG513210 and NAG5-9670, and STScI grant AR-09524.01A. P.D. acknowledges grants from PAPIIT, UNAM, and CONACyT, Mexico. This publication makes use of data products from the Two Micron All Sky Survey, which is a joint project of the University of Massachusetts and the Infrared Processing and Analysis Center/California Institute of Technology, funded by the National Aeronautics and Space Administration and the National Science Foundation. It has also made use of the SIMBAD and VizieR databases, operated at CDS (Strasbourg, France), NASA's Astrophysics Data System Abstract Service, and of the NASA/ IPAC Infrared Science Archive operated by JPL, California Institute of Technology (Caltech), under contract with NASA.

Facilities: Spitzer(IRS)

\section{REFERENCES}

Adams, F. C., \& Shu, F. H. 1986, ApJ, 308, 836

Adams, F. C., Lada, Ch. J., \& Shu, F. H. 1987, ApJ, 312, 788

Adams, F. C., Emerson, J. P., \& Fuller, G. A. ApJ, 357, 606

Alexander, R. D., Casali, M. M., André, P., Persi, P., \& Eiroa, C. 2003, A\&A, 401, 613

André, P., Ward-Thompson, D., \& Barsony, M. 1993, ApJ, 406, 122

André, P., \& Montmerle, T. 1994, ApJ, 420, 837

Andrews, S. M., \& Williams, J. P. 2005, ApJ, 631, 1134

Andrews, S. M., \& Williams, J. P. 2007, ApJ, 659, 705

Apai, D., Tóth, L. V., Henning, Th., Vavrek, R., Kovács, Z., \& Lemke, D. 2005, A\&A, 433, L33

Arce, H. G., \& Goodman, A. A. 2002, ApJ, 575, 928

Barsony, M., \& Kenyon, S. J. 1992, ApJ, 384, L53

Beckwith, S. V. W., Sargent, A. I., Chini, R. S., \& Güsten, R. 1990, AJ, 99, 924

Beichman, C. A., Myers, P. C., Emerson, J. P., Harris, S., Mathieu, R., Benson, P. J., \& Jennings, R. E. 1986, ApJ, 307, 337

Bertout, C., Robichon, N., \& Arenou, F. 1999, A\&A, 352, 574

Bontemps, S., André, P., Terebey, S., \& Cabrit, S. 1996, A\&A, 311,858

Bowey J. E., Adamson, A. J., \& Yates, J. A. 2003, MNRAS, 340, 1173

Briceño, C., Luhman, K. L., Hartmann, L., Stauffer, J. R., \& Kirkpatrick, J. D. 2002, ApJ, 580, 317

Burrows, Ch. J., Stapelfeldt, K. R., Watson, A. M., Krist, J. E., Ballester, G. E., Clarke, J. T., Crisp, D., Gallagher, J. S. III, et al. 1996, ApJ, 473, 437

Calvet, N., Hartmann, L., Kenyon, S. J., \& Whitney, B. A. 1994, ApJ, 434, 330

Calvet, N., Muzerolle, J., Briceño, C., Hernández, J., Hartmann, L., Saucedo, J. L., \& Gordon, K. D. 2004, AJ, 128, 1294
Campbell, B., Persson, S. E., Strom, S. E., \& Grasdalen, G. L. 1988, AJ, 95, 1173

Chandler, C. J., Terebey, S., Barsony, M., Moore, T. J. T., \& Gautier, T. N. 1996, ApJ, 471, 308

Chandler, C. J., Barsony, M., \& Moore, T. J. T. 1998, MNRAS, 299,789

Chandler, C. J., \& Richer, J. S. 2000, ApJ, 530, 851

Chen, H., Myers, P. C., Ladd, E. F., \& Wood, D. O. S. 1995 ApJ, 445, 377

Chini, R., Ward-Thompson, D., Kirk, J. M., Nielbock, M., Reipurth, B., \& Sievers, A. 2001, A\&A, 369, 155

Close, L. M., Roddier, F., Northcott, M. J., Roddier, C., \& Graves, J. E. 1997, ApJ, 478, 766

Cohen, M., \& Schwartz, R. D. 1987, ApJ, 316, 311

Cohen, M., Megeath, S. T., Hammersley, P. L., Martín-Luis, F., \& Stauffer, J. 2003, AJ, 125, 2645

Cotera, A. S., Whitney, B. A., Young, E., Wolff, M. J., Wood, K., Povich, M., Schneider, G., Rieke, M., \& Thompson, R. 2001 ApJ, 556, 958

D'Alessio, P., Calvet, N., \& Hartmann, L. 1997, ApJ, 474, 397

D’Alessio, P., Calvet, N., Hartmann, L., Lizano, S., \& Cantó, J. 1999, ApJ, 527, 893

Devine, D., Reipurth, B., Bally, J., \& Balonek, Th. J. 1999, AJ, 117,2931

Draine, B. T., \& Lee, H. M. 1984, ApJ, 285, 89

Duchêne, G., Bouvier, J., Bontemps, S., André, P., \& Motte, F. 2004, A\&A, 427, 651

Dutrey, A., Guilloteau, S., Duvert, G., Prato, L., Simon, M., Schuster, K., \& Ménard, F. 1996, A\&A, 309, 493

Ehrenfreund, P., Boogert, A. C. A., Gerakines, P. A., Jansen, D. J., Schutte, W. A., Tielens, A. G. G. M., \& van Dishoeck, E. F. 1996, A\&A, 315, L341 
Ehrenfreund, P., Kerkhof, O., Schutte, W. A., Boogert, A. C. A., Gerakines, P. A., Dartois, E., d'Hendecourt, L., Tielens, A. G. G. M., et al. 1999, A\&A, 350, 240

Eislöffel, J., \& Mundt, R. 1998, ApJ, 115, 1554

Eisner, J. A., Hillenbrand, L. A., Carpenter, J. M., \& Wolf, S. 2005, ApJ, 635, 396

Fuller, G. A., Ladd, E. F., \& Hodapp, K.-W. 1996, ApJ, 463, L97

Furlan, E., Hartmann, L., Calvet, N., D'Alessio, P., Franco-Hernández, R., Forrest, W. J., Watson, D. M., Uchida, K. I., et al. 2006, ApJS, 165, 568

Gibb, E. L., Whittet, D. C. B., Boogert, A. C. A., \& Tielens, A. G. G. M. 2004, ApJS, 151, 35

Gomez, M., Whitney, B. A., \& Kenyon, S. J. 1997, AJ, 114, 1138

Gramajo, L. V., Whitney, B. A., Kenyon, S. J., Gómez, M., \& Merrill, K. M. 2007, AJ, 133, 1911

Green, J. D., Hartmann, L., Calvet, N., Watson, D. M., Ibrahimov, M., Furlan, E., Sargent, B., \& Forrest, W. J. 2006, ApJ, 648, 1099

Gullbring, E., Hartmann, L., Briceño, C., \& Calvet, N. 1998, ApJ, 492, 323

Hartigan, P., Edwards, S., \& Ghandour, L. 1995, ApJ, 452, 736

Hartmann, L., Boss, A., Calvet, N., \& Whitney, B. 1994, ApJ, 430, L49

Hartmann, L., \& Kenyon, S. J. 1996, ARA\&A, 34, 207

Hartmann, L., Calvet, N., \& Boss, A. 1996, ApJ, 464, 387

Hartmann, L., Calvet, N., Allen, L., Chen, H., \& Jayawardhana, R. 1999, AJ, 118, 1784

Hartmann, L., Megeath, S. T., Allen, L., Luhman, K., Calvet, N., D'Alessio, P., Franco-Hernández, R., \& Fazio, G. 2005, ApJ 629,881

Heyer, M. H., Snell, R. L., Goldsmith, P. F., \& Myers, P. C. 1987, ApJ, 321, 370

Higdon, S. J. U., et al. 2004, PASP, 116, 975

Hogerheijde, M. R., van Dishoeck, E. F., Blake, G. A., \& van Langevelde, H. J. 1998, ApJ, 502, 315

Houck, J. R., et al. 2004, ApJS, 154, 18

Jayawardhana, R., Hartmann, L., \& Calvet, N. 2001, ApJ, 548, 310

Kenyon, S. J., Hartmann, L. W., Strom, K. M., \& Strom, S. E. 1990, AJ, 99, 869

Kenyon, S. J., Calvet, N., \& Hartmann, L. 1993a, ApJ, 414, 676

Kenyon, S. J., Whitney, B. A., Gomez, M., \& Hartmann, L. 1993, ApJ, 414, 773

Kenyon, S. J., Dobrzycka, D., \& Hartmann, L. 1994b, AJ, 108, 1872

Kenyon, S. J., \& Hartmann, L. 1995, ApJS, 101, 117 (KH95)

Kenyon, S. J., Brown, D. I., Tout, Ch. A., \& Berlind, P. 1998, AJ, 115,2491

Koresko, C. D., Herbst, T. M., \& Leinert, Ch. 1997, ApJ, 480, 741

Lada, Ch. J., \& Wilking, B. A. 1984, ApJ, 287, 610

Lada, Ch. J. 1987, in Star Forming Regions, proceedings of the IAU Symposium No. 115, ed. M. Peimbert \& J. Jugaku, Dordrecht:Reidel, 1

Ladd, E. F., Adams, F. C., Casey, S., Davidson, J. A., Fuller, G. A., Harper, D. A., Myers, P. C., \& Padman, R. 1991, ApJ, 382, 555

Leinert, Ch., \& Haas, M. 1989, ApJ, 342, L39

Loinard, L., Rodríguez, L. F., D'Alessio, P., Wilner, D. J., \& Ho, P. T. P. 2002, ApJ, 581, L109

Looney L. W., Mundy, L. G., \& Welch, W. J. 1997, ApJ, 484, L157

Lucas, P. W., \& Roche, P. F. 1997, MNRAS, 286, 895

Lucas, P. W., \& Roche, P. F. 1998, MNRAS, 299, 723

Luhman, K. L. 2000, ApJ, 544, 1044

Luhman, K. L. 2006, ApJ, 645, 676

Luhman, K. L., Whitney, B. A., Meade, M. R., Babler, B. L., Indebetouw, R., Bracker, S., \& Churchwell, E. B. 2006, ApJ, 647,1180

Mathis, J. S., Rumpl, W., \& Nordsieck, K. H. 1977, ApJ, 217, 425

Ménard, F., Monin, J.-L., Angelucci, F., \& Rouan, D. 1993, ApJ, 414, L117

Ménard, F., Dougados, C., Magnier, E., Cuillandre, J.-C., Fahlman, G., Forveille, T., Lai, O., Manset, N., et al. 2001, BAAS, 198, 4803

Mitchell, G. F., Sargent, A. I., \& Mannings, V. 1997, ApJ, 483, L127

Mitchell, R. M., \& Robinson, G. 1981, MNRAS, 196, 801
Monin, J.-L., Pudritz, R. E., \& Lazareff, B. 1996, A\&A, 305, 572 Moriarty-Schieven, G. H., Wannier, P. G., Tamura, M., \& Keene, J. 1992, ApJ, 400, 260

Moriarty-Schieven, G. H., Wannier, P. G., Keene, J., \& Tamura, M. 1994, ApJ, 436, 800

Motte, F., \& André, P. 2001, A\&A, 365, 440

Muzerolle, J., Hartmann, L., \& Calvet, N. 1998, AJ, 116, 2965

Muzerolle, J., Hillenbrand, L., Calvet, N., Briceño, C., Hartmann, L. 2003a, ApJ, 592, 266

Myers, P. C., Fuller, G. A., Mathieu, R. D., Beichman, C. A., Benson, P. J., Schild, R. E., \& Emerson, J. P. 1987, ApJ, 319, 340

Ohashi, N., Hayashi, M., Kawabe, R., \& Ishiguro, M. 1996, ApJ, 466,317

Ohashi, N., Hayashi, M., Ho, P. T. P., \& Momose, M. 1997a, ApJ, 475, 211

Ohashi, N., Hayashi, M., Ho, P. T. P., Momose, M., Tamura, M., Hirano, N., \& Sargent, A. 1997b, ApJ, 488, 317

Onishi, T., Mizuno, A., Kawamura, A., Ogawa, H., \& Fukui, Y. 1998, ApJ, 502, 296

Onishi, T., Mizuno, A., Kawamura, A., Tachihara, K., \& Fukui, Y. 2002, ApJ, 575, 950

Osorio, M., D'Alessio, P., Muzerolle, J., Calvet, N., \& Hartmann, L. 2003, ApJ, 586, 1148

Osterloh, M., \& Beckwith, S. V. W. 1995, ApJ, 439, 288

Padgett, D. L., Brandner, W., Stapelfeldt, K. R., Strom, S. E.,

Terebey, S., \& Koerner, D. 1999, AJ, 117, 1490

Park, S., \& Kenyon, S. J. 2002, AJ, 123, 3370

Pollack, J. B., Hollenbach, D., Beckwith, S., Simonelli, D. P., Roush, T., \& Fong, W. 1994, ApJ, 421, 615

Reipurth, B., Chini, R., Krügel, E., Kreysa, E., \& Sievers, A. 1993, A\&A, 273, 221

Reipurth, B., Yu, K. C., Heathcote, S., Bally, J., \& Rodríguez, L. F. 2000, AJ, 120, 1449

Rodríguez, L. F., D'Alessio, P., Wilner, D. J., Ho, P. T. P., Torrelles, J. M., Curiel, S., Gómez, Y., Lizano, S., et al. 1998, Nature, 395, 355

Sargent, A. I., \& Beckwith, S. V. W. 1991, ApJ, 382, L31

Shu, F., Najita, J., Ostriker, E., Wilkin, F., Ruden, S., \& Lizano, S. 1994, ApJ, 429, 781

Shuping, R. Y., Chiar, J. E., Snow, Th. P., \& Kerr, Th. 2001, ApJ, 547, L161

Snell, R. L., \& Schloerb, F. P. 1985, ApJ, 295, 490

Stapelfeldt, K. R., Burrows, Ch. J., Krist, J. E., Trauger, J. T., Hester, J. J., Holtzman, J. A., Ballester, G. E., Casertano, S., et al. 1995, ApJ, 449, 888

Stapelfeldt, K. R., et al. 1997, in IAU Symp. 182, Herbig-Haro Flows and the Birth of Stars, ed. B. Reipurth \& C. Bertout (Dordrecht: Kluwer), 355

Stark, D. P., Whitney, B. A., Stassun, K., \& Wood, K. 2006, ApJ, 649, 900

Strom, K. M., \& Strom, S. E. 1994, ApJ, 424, 237

Tafalla, M., Santiago, J., Johnstone, D., \& Bachiller, R. 2004, A\&A, 423, L21

Tamura, M., Gatley, I., Waller, W., \& Werner, M. W. 1991, ApJ, 374, L25

Tamura, M., Ohashi, N., Hirano, N., Itoh, Y., \& Moriarty-Schieven, G. H. 1996, AJ, 112, 2076

Tegler, S. C., Weintraub, D. A., Rettig, T. W., Pendleton, Y. J., Whittet, D. C. B., \& Kulesa, C. A. 1995, ApJ, 439, 279

Terebey, S., Shu, F. H., \& Cassen, P. 1984, ApJ, 286, 529 (TSC)

Terebey, S., van Buren, D., Padgett D. L., Hancock, T., \& Brundage, M. 1998, ApJ, 507, L71

Terebey, S., van Buren, D., Matthews, K., \& Padgett, D. L. 2000, AJ, 119, 2341

Terebey, S., van Buren, D., Brundage, M., \& Hancock, T. 2006, ApJ, 637, 811

van Dishoeck, E. F. 2004, ARA\&A, 42, 119

Watson, D. M., Kemper, F., Calvet, N., Keller, L. D., Furlan, E., Hartmann, L., Forrest, W. J., Chen, C. H., et al. 2004, ApJS, 154,391

Weaver, W. B., \& Jones, G. 1992, ApJS, 78, 239

Weingartner, J. C., \& Draine, B. T. 2001, ApJ, 548, 296

Welch, Wm. J., Hartmann, L., Helfer, T., \& Briceño, C. 2000, ApJ, 540, 362

Werner, M. W., et al. 2004, ApJS, 154, 1 
White, G. J., Liseau, R., Men'shchikov, A. B., Justtanont, K.,

Nisini, B., Benedettini, M., Caux, E., Ceccarelli, C., et al. 2000, A\&A, 364, 741

White, R. J., \& Ghez, A. M. 2001, ApJ, 556, 265

White, R. J., \& Hillenbrand, L. A. 2004, ApJ, 616, 998

Whitney, B. A., Kenyon, S. J., \& Gómez, M. 1997, ApJ, 485, 703

Wolf, S., Padgett, D. L., \& Stapelfeldt, K. R. 2003, ApJ, 588, 373
Wood, K., Kenyon, S. J., Whitney, B., \& Turnbull, M. 1998, ApJ, 497, 404

Young, C. H., Shirley, Y. L., Evans, N. J. II, \& Rawlings, J. M. C. 2003, ApJS, 145, 111 
TABLE 1

Properties of Observed Class I Objects

\begin{tabular}{|c|c|c|c|c|c|}
\hline $\begin{array}{c}\text { Name } \\
\text { (1) }\end{array}$ & $\begin{array}{l}\text { Alt. name } \\
\text { (2) }\end{array}$ & $\begin{array}{c}\text { Multiplicity }^{a} \\
\text { (3) }\end{array}$ & $\begin{array}{l}\text { Spectral } \\
\text { Type } \\
(4)\end{array}$ & $\begin{array}{c}\mathrm{L}_{b o l} \\
\left(L_{\odot}\right) \\
(5)\end{array}$ & $\begin{array}{c}\text { References } \\
\text { (6) }\end{array}$ \\
\hline $04016+2610$ & L1489 IRS & $\mathrm{s}$ & K4 & 3.7 & 1,2 \\
\hline $04108+2803 \mathrm{~B}^{b}$ & L1495 IRS & $21.2^{\prime \prime}$ & $\cdots$ & 0.62 & 2 \\
\hline $04154+2823$ & $\ldots$ & $\mathrm{s}$ & M0.5-M4.5 & 0.33 & 3,4 \\
\hline $04158+2805$ & $\cdots$ & $\mathrm{s}$ & M5-M6 & 0.20 & $1,5,6$ \\
\hline $04166+2706$ & $\cdots$ & $\mathrm{s}$ & $\ldots$ & 0.5 & 7 \\
\hline $04169+2702$ & $\cdots$ & s & $\cdots$ & 0.8 & 2 \\
\hline $04181+2654 \mathrm{~A}^{d}$ & $\cdots$ & $31.3^{\prime \prime}$ & $\cdots$ & 0.26 & 2 \\
\hline $04181+2654 \mathrm{~B}^{d}$ & $\cdots$ & $31.3^{\prime \prime}$ & $\cdots$ & 0.25 & 2 \\
\hline $04239+2436(\mathrm{~A}, \mathrm{~B})$ & $\cdots$ & $0.30^{\prime \prime}$ & $\cdots$ & 1.27 & 8,2 \\
\hline $04248+2612(\mathrm{~A}, \mathrm{~B}, \mathrm{C})^{c}$ & HH31 IRS2 & $0.16^{\prime \prime}, 4.55^{\prime \prime}$ & M4-M5 & 0.36 & $9,10,1,5,2$ \\
\hline $04264+2433$ & Elias 6 & $\mathrm{~s}$ & M1 & 0.37 & 1,2 \\
\hline $04278+2253(\mathrm{~A}, \mathrm{~B})$ & $\cdots$ & $6.8^{\prime \prime}$ & $\mathrm{G} 8+\mathrm{K} 7$ & 7.2 & 1,6 \\
\hline $04295+2251$ & L1536 IRS & $\mathrm{s}$ & $\cdots$ & 0.44 & 2 \\
\hline $04302+2247$ & $\ldots$ & $\mathrm{s}$ & $\cdots$ & 0.34 & 2 \\
\hline $04325+2402(\mathrm{~A}, \mathrm{~B}, \mathrm{C})^{e}$ & L1535 IRS & $0.24^{\prime \prime}, 8.15^{\prime \prime}$ & $\ldots$ & 0.9 & $11,10,12$ \\
\hline $04361+2547(\mathrm{~A}, \mathrm{~B})$ & TMR 1 & $0.31^{\prime \prime}$ & $\cdots$ & 3.8 & 14,6 \\
\hline $04365+2535$ & TMC 1A & $\mathrm{s}$ & $\cdots$ & 2.4 & 4 \\
\hline $04368+2557(\mathrm{~A}, \mathrm{~B})$ & L1527 IRS & $0.17^{\prime \prime}$ & $\cdots$ & 1.6 & 15,6 \\
\hline $04381+2540(\mathrm{~A}, \mathrm{~B})$ & TMC 1 & $0.6^{\prime \prime}$ & $\cdots$ & 0.73 & 13,4 \\
\hline $04489+3042$ & $\cdots$ & $\mathrm{s}$ & M3-M4 & 0.30 & $5,1,2$ \\
\hline $\mathrm{CoKu} \mathrm{Tau/1} \mathrm{(A,B)}$ & $\cdots$ & $0.24^{\prime \prime}$ & $\mathrm{K} 7+\mathrm{M}$ & $>0.29$ & $9,1,2$ \\
\hline DG Tau B & $\cdots$ & $\mathrm{s}$ & $\ldots$ & $>0.02$ & 2 \\
\hline GV Tau (A,B) & Haro 6-10 & $1.3^{\prime \prime}$ & K7 & 6.98 & $10,1,2$ \\
\hline $\mathrm{HH} 30$ & $\cdots$ & $\mathrm{s}$ & M0 & $>0.1$ & 1,18 \\
\hline HL Tau & $04287+1807$ & $\mathrm{~s}$ & K5 & 6.60 & 1,2 \\
\hline IC 2087 IR & $04369+2539$ & $\mathrm{~s}$ & $\mathrm{~K} 4$ & 3.80 & 1,2 \\
\hline L1551 IRS5 (A,B) & $04287+1801$ & $0.3^{\prime \prime}$ & G-K & 28 & $16,17,12$ \\
\hline LkHa 358 & $\mathrm{CoKu} \mathrm{Tau} / 2$ & $\mathrm{~s}$ & M5.5 & 0.59 & 3 \\
\hline
\end{tabular}

REFERENCES. - (1) White \& Hillenbrand (2004); (2) Kenyon \& Hartmann (1995); (3) Luhman (2000); (4) Mvers et al. (1987); (5) Luhman (2006); (6) Kenvon et al. (1990); (7) Young et al. (2003); (8) Reipurth et al. (2000); (9) Padgett et al. (1999); (10) Duchêne et al. (2004); (11) Hartmann et al. (1999); (12) Motte \& André (2001); (13) Apai et al. (2005); (14) Tereber et al. (1998); (15) Loinard et al. (2002); (16) Rodríguez et al. (1998); (17) Kenvon et al. (1998); (18) Reipurth et al. (1993)

Note. - Column (1) gives the name of the object, column (2) other object names commonly found in the literature, column (3) the multiplicity of the object (see note (a) below), column (4) the spectral type, column (5) the luminosity of the source taken from the literature, and column (6) gives the references for the data listed in the previous columns.

a "s" means single star; for multiple systems, the separation between the components in arcseconds is listed.

${ }^{b} 04108+2803 \mathrm{~B}$ is separated by $21^{\prime \prime}$ from $04108+2803 \mathrm{~A}$.

${ }^{c} 04248+2612 \mathrm{~A}$ and B are separated by $0.16^{\prime \prime}$ (Padgett et al. 1999); a third component was detected by Duchêne et al. (2004) at a distance of $4.55^{\prime \prime}$ and a position angle of $15^{\circ}$.

$d^{2} 31.3^{\prime \prime}$ is the separation between $04181+2654 \mathrm{~A}$ and $\mathrm{B}$.

e $04325+2402$ is a possible triple system; the sub-arsecond binary may just be a single source surrounded by a complex reflection nebula (Hartmann et al. 1999). 
Furlan et al.

TABLE 2

Long-Wavelength Data of Class I Objects

\begin{tabular}{|c|c|c|c|c|}
\hline Name & $\begin{array}{c}\lambda \\
(\mu \mathrm{m})\end{array}$ & $\begin{array}{l}\text { Flux } \\
(\mathrm{Jy})\end{array}$ & $\begin{array}{l}\text { Aperture size } \\
\text { (FWHM) }\end{array}$ & Reference \\
\hline \multirow{7}{*}{$04016+2610$} & 160 & 46.0 & $50^{\prime \prime}$ & 1 \\
\hline & 350 & 12.48 & $9^{\prime \prime}$ & 2 \\
\hline & 450 & 4.23 & $40^{\prime \prime}$ & 4 \\
\hline & 800 & 0.58 & $16 !^{\prime \prime} 8$ & 5 \\
\hline & 850 & 0.59 & $40^{\prime \prime}$ & 4 \\
\hline & 1100 & 0.18 & $18 .^{\prime \prime} 5$ & 5 \\
\hline & 1300 & 0.15 & $60^{\prime \prime}$ & 6 \\
\hline \multirow[t]{5}{*}{$04108+2803 \mathrm{~B}$} & 450 & 1.13 & $40^{\prime \prime}$ & 4 \\
\hline & 800 & 0.085 & $16 !^{\prime \prime} 8$ & 5 \\
\hline & 850 & 0.17 & $40^{\prime \prime}$ & 4 \\
\hline & 1100 & $<0.1$ & $18 . .5$ & 5 \\
\hline & 1300 & 0.04 & $60^{\prime \prime}$ & 6 \\
\hline \multirow[t]{5}{*}{$04154+2823$} & 350 & 0.44 & $9^{\prime \prime}$ & 2 \\
\hline & 450 & 0.495 & $9^{\prime \prime}$ & 2 \\
\hline & 800 & $<0.1$ & $16 !^{\prime \prime} 8$ & 5 \\
\hline & 850 & 0.14 & $15^{\prime \prime}$ & 2 \\
\hline & 1100 & $<0.1$ & $18 . .5$ & 5 \\
\hline \multirow{2}{*}{$04158+2805$} & 880 & 0.067 & $\sim 5^{\prime \prime}$ & 12 \\
\hline & 1300 & 0.11 & $60^{\prime \prime}$ & 6 \\
\hline \multirow[t]{6}{*}{$04166+2706$} & 350 & 6.94 & $9^{\prime \prime}$ & 2 \\
\hline & 450 & 4.2 & $40^{\prime \prime}$ & 4 \\
\hline & 800 & 0.65 & $16^{\prime \prime} 8$ & 3 \\
\hline & 850 & 1.08 & $40^{\prime \prime}$ & 4 \\
\hline & 1100 & 0.30 & $18^{\prime \prime} 5$ & 3 \\
\hline & 1300 & 0.80 & $60^{\prime \prime}$ & 6 \\
\hline \multirow[t]{6}{*}{$04169+2702$} & 350 & 7.34 & $9^{\prime \prime}$ & 2 \\
\hline & 450 & 6.09 & $40^{\prime \prime}$ & 4 \\
\hline & 800 & 0.75 & $16^{\prime \prime} 8$ & 5 \\
\hline & 850 & 1.14 & $40^{\prime \prime}$ & 4 \\
\hline & 1100 & 0.28 & $18^{\prime \prime} 5$ & 5 \\
\hline & 1300 & 0.73 & $60^{\prime \prime}$ & 6 \\
\hline $04181+2654 \mathrm{~A}$ & 1300 & 0.23 & $60^{\prime \prime}$ & 6 \\
\hline $04181+2654 \mathrm{~B}$ & $\ldots$ & $\ldots$ & $\ldots$ & $\ldots$ \\
\hline \multirow[t]{6}{*}{$04239+2436(\mathrm{~A}, \mathrm{~B})$} & 350 & 1.14 & $9^{\prime \prime}$ & 2 \\
\hline & 450 & $<0.66$ & $9^{\prime \prime}$ & 2 \\
\hline & 800 & 0.33 & $16 !^{\prime \prime} 8$ & 5 \\
\hline & 850 & 0.21 & $15^{\prime \prime}$ & 2 \\
\hline & 1100 & 0.11 & $18 !^{\prime \prime} 5$ & 5 \\
\hline & 1300 & 0.17 & $60^{\prime \prime}$ & 6 \\
\hline \multirow[t]{6}{*}{$04248+2612(\mathrm{~A}, \mathrm{~B}, \mathrm{C})$} & 350 & 1.18 & $9^{\prime \prime}$ & 2 \\
\hline & 450 & 2.96 & $40^{\prime \prime}$ & 4 \\
\hline & 800 & 0.25 & $16 !^{\prime \prime} 8$ & 5 \\
\hline & 850 & 0.56 & $40^{\prime \prime}$ & 4 \\
\hline & 1100 & 0.10 & $18 . .5$ & 5 \\
\hline & 1300 & 0.45 & $60^{\prime \prime}$ & 6 \\
\hline \multirow[t]{3}{*}{$04264+2433$} & 450 & 0.63 & $40^{\prime \prime}$ & 4 \\
\hline & 850 & 0.13 & $40^{\prime \prime}$ & 4 \\
\hline & 1300 & 0.03 & $60^{\prime \prime}$ & 6 \\
\hline \multirow[t]{2}{*}{$04278+2253(\mathrm{~A}, \mathrm{~B})$} & 450 & $<0.69$ & $9^{\prime \prime}$ & 2 \\
\hline & 850 & 0.04 & $15^{\prime \prime}$ & 2 \\
\hline $04295+2251$ & 350 & 1.34 & $9^{\prime \prime}$ & 2 \\
\hline & 450 & 2.66 & $40^{\prime \prime}$ & 4 \\
\hline & 800 & 0.24 & $16 !^{\prime \prime} 8$ & 5 \\
\hline & 850 & 0.42 & $40^{\prime \prime}$ & 4 \\
\hline & 1100 & 0.09 & $18 . .5$ & 5 \\
\hline & 1300 & 0.115 & $60^{\prime \prime}$ & 6 \\
\hline $04302+2247$ & 350 & 2.87 & $9^{\prime \prime}$ & 2 \\
\hline & 450 & 2.09 & $40^{\prime \prime}$ & 4 \\
\hline & 800 & 0.34 & $16 !^{\prime \prime} 8$ & 5 \\
\hline & 850 & 0.57 & $40^{\prime \prime}$ & 4 \\
\hline & 1100 & 0.15 & $188^{\prime \prime} 5$ & 5 \\
\hline & 1300 & 0.18 & $60^{\prime \prime}$ & 6 \\
\hline $04325+2402(\mathrm{~A}, \mathrm{~B}, \mathrm{C})$ & 160 & 38.0 & $50^{\prime \prime}$ & 1 \\
\hline & 450 & 0.61 & $9^{\prime \prime}$ & 2 \\
\hline & 800 & 0.30 & $16 !^{\prime \prime} 8$ & 5 \\
\hline & 850 & 0.19 & $15^{\prime \prime}$ & 2 \\
\hline & 1100 & 0.07 & $18{ }^{\prime \prime} 5$ & 5 \\
\hline & 1300 & 0.52 & $60^{\prime \prime}$ & 6 \\
\hline $04361+2547(\mathrm{~A}, \mathrm{~B})$ & 450 & 2.35 & $40^{\prime \prime}$ & 4 \\
\hline & 800 & 0.63 & $16 !^{\prime \prime} 8$ & 5 \\
\hline & 850 & 0.64 & $40^{\prime \prime}$ & 4 \\
\hline & 1100 & 0.19 & $18 . .5$ & 5 \\
\hline
\end{tabular}


Spitzer Spectra and Models of Protostars in Taurus

TABLE 2 - Continued

\begin{tabular}{|c|c|c|c|c|}
\hline Name & $\begin{array}{c}\lambda \\
(\mu \mathrm{m})\end{array}$ & $\begin{array}{l}\text { Flux } \\
(\mathrm{Jy})\end{array}$ & $\begin{array}{l}\text { Aperture size } \\
\text { (FWHM) }\end{array}$ & Reference \\
\hline & 1300 & 0.44 & $60^{\prime \prime}$ & 6 \\
\hline \multirow{6}{*}{$04365+2535$} & 350 & 20.6 & $45^{\prime \prime}$ & 10 \\
\hline & 450 & 12.7 & $45^{\prime \prime}$ & 10 \\
\hline & 800 & 1.01 & $16 !^{\prime \prime} 8$ & 5 \\
\hline & 850 & 1.80 & $45^{\prime \prime}$ & 10 \\
\hline & 1100 & 0.44 & $18^{\prime \prime} 5$ & 5 \\
\hline & 1300 & 0.45 & $60^{\prime \prime}$ & 6 \\
\hline \multirow{7}{*}{$04368+2557(\mathrm{~A}, \mathrm{~B})$} & 160 & 69.0 & $50^{\prime \prime}$ & 1 \\
\hline & 350 & 12.0 & $45^{\prime \prime}$ & 1 \\
\hline & 450 & 2.85 & $9^{\prime \prime}$ & 2 \\
\hline & 800 & 1.52 & $16 !^{\prime \prime} 8$ & 5 \\
\hline & 850 & 0.895 & $15^{\prime \prime}$ & 2 \\
\hline & 1100 & 0.48 & $18^{\prime \prime} 5$ & 5 \\
\hline & 1300 & 1.50 & $60^{\prime \prime}$ & 6 \\
\hline \multirow{5}{*}{$04381+2540(\mathrm{~A}, \mathrm{~B})$} & 450 & 2.82 & $40^{\prime \prime}$ & 4 \\
\hline & 800 & 0.29 & $16 !^{\prime \prime} 8$ & 5 \\
\hline & 850 & 0.56 & $40^{\prime \prime}$ & 4 \\
\hline & 1100 & 0.12 & $18^{\prime \prime} 5$ & 5 \\
\hline & 1300 & 0.30 & $60^{\prime \prime}$ & 6 \\
\hline $04489+3042$ & 1300 & $\gtrsim 0.015$ & $60^{\prime \prime}$ & 6 \\
\hline \multirow[t]{3}{*}{$\mathrm{CoKu} \mathrm{Tau} / 1(\mathrm{~A}, \mathrm{~B})$} & 450 & $<0.52$ & $9^{\prime \prime}$ & 2 \\
\hline & 850 & 0.035 & $15^{\prime \prime}$ & 2 \\
\hline & 1300 & $<0.012$ & $11^{\prime \prime}$ & 9 \\
\hline DG Tau B & 1300 & 0.31 & $\ldots$ & 11 \\
\hline \multirow{6}{*}{ GV Tau $(\mathrm{A}, \mathrm{B})$} & 350 & 1.68 & $9^{\prime \prime}$ & 2 \\
\hline & 450 & 1.81 & $9^{\prime \prime}$ & 2 \\
\hline & 800 & 0.57 & $16^{\prime \prime}$ & 8 \\
\hline & 850 & 0.28 & $15^{\prime \prime}$ & 2 \\
\hline & 1100 & 0.18 & $19^{\prime \prime}$ & 8 \\
\hline & 1300 & 0.20 & $60^{\prime \prime}$ & 6 \\
\hline HH 30 & 1300 & 0.035 & $60^{\prime \prime}$ & 6 \\
\hline \multirow[t]{6}{*}{ HL Tau } & 350 & 26.4 & $45^{\prime \prime}$ & 10 \\
\hline & 450 & 16.8 & $45^{\prime \prime}$ & 10 \\
\hline & 800 & 2.58 & $15 !^{\prime \prime} 8$ & 7 \\
\hline & 850 & 2.97 & $45^{\prime \prime}$ & 10 \\
\hline & 1100 & 1.11 & $18 !^{\prime \prime} 4$ & 7 \\
\hline & 1300 & 1.20 & $60^{\prime \prime}$ & 6 \\
\hline \multirow[t]{2}{*}{ IC 2087 IR } & 450 & 1.365 & $9^{\prime \prime}$ & 2 \\
\hline & 850 & 0.50 & $15^{\prime \prime}$ & 2 \\
\hline \multirow{6}{*}{ L1551 IRS5 $(\mathrm{A}, \mathrm{B})$} & 350 & 164 & $45^{\prime \prime}$ & 10 \\
\hline & 450 & 94 & $45^{\prime \prime}$ & 10 \\
\hline & 800 & 8.05 & $16 !^{\prime \prime} 8$ & 5 \\
\hline & 850 & 12.1 & $45^{\prime \prime}$ & 10 \\
\hline & 1100 & 2.77 & $18 . .5$ & 5 \\
\hline & 1300 & 3.40 & $60^{\prime \prime}$ & 6 \\
\hline LkHa 358 & 1300 & 0.032 & $11^{\prime \prime}$ & 9 \\
\hline
\end{tabular}

References. - (1) Ladd et al. (1991); (2) Andrews \& Williams (2005); (3) Barsony \& Kenyon (1992); (4) Young et al. (2003); (5) Moriarty-Schieven et al. (1994); (6) Motte \& André (2001); (7) Adams et al. (1990); (8) Chandler et al. (1998); (9) Osterloh \& Beckwith (1995); (10) Chandler \& Richer (2000); (11) Padgett et al. (1999); (12) Andrews \& Williams (2007) 
TABLE 3

Model Fits for Class I Objects

\begin{tabular}{|c|c|c|c|c|c|c|c|c|c|c|}
\hline $\begin{array}{c}\text { Name } \\
(1)\end{array}$ & $\begin{array}{c}\mathrm{L} \\
\left(L_{\odot}\right) \\
(2)\end{array}$ & $\begin{array}{c}\rho_{1} \\
\left(\mathrm{~g} \mathrm{~cm}^{-3}\right) \\
(3)\end{array}$ & $\begin{array}{c}R_{c} \\
(\mathrm{AU}) \\
(4)\end{array}$ & $\begin{array}{c}\mathrm{R}_{\text {diskmin }} \\
\left(\mathrm{R}_{\text {star }}\right) \\
(5)\end{array}$ & $\begin{array}{c}\mathrm{R}_{\max } \\
(\mathrm{AU}) \\
(6)\end{array}$ & $\begin{array}{l}\eta \\
(7)\end{array}$ & $\begin{array}{r}\eta_{\text {star }} \\
(8)\end{array}$ & $\begin{array}{c}\mathrm{CO}_{2} \text { ice } \\
\text { abundance } \\
(9)\end{array}$ & $\begin{array}{c}\theta \\
(10)\end{array}$ & $\begin{array}{r}\mathrm{i} \\
(11)\end{array}$ \\
\hline $04016+2610$ & 4.5 & $4.5 \times 10^{-14}$ & 100 & 1 & 6000 & 1.0 & 0.1 & $1.5 \times 10^{-4}$ & $5^{\circ}$ & $40^{\circ}$ \\
\hline $04108+2803 \mathrm{~B}$ & 0.7 & $1.5 \times 10^{-14}$ & 40 & 3 & 6000 & TSC & 0.3 & $1.0 \times 10^{-4}$ & $10^{\circ}$ & $40^{\circ}$ \\
\hline $04154+2823$ & 0.35 & $7.0 \times 10^{-15}$ & 10 & 1 & 5000 & TSC & 0.1 & $1.0 \times 10^{-4}$ & $5^{\circ}$ & $20^{\circ}$ \\
\hline $04158+2805$ & 0.3 & $2.0 \times 10^{-14}$ & 60 & 3 & 5000 & TSC & 0.7 & $4.0 \times 10^{-5}$ & $5^{\circ}$ & $30^{\circ}$ \\
\hline $04166+2706$ & 0.6 & $4.5 \times 10^{-14}$ & 300 & 5 & 10000 & TSC & 0.2 & $2.0 \times 10^{-5}$ & $6^{\circ}$ & $85^{\circ}$ \\
\hline $04169+2702$ & 1.5 & $3.2 \times 10^{-14}$ & 100 & 1 & 10000 & 1.0 & 0.8 & $6.0 \times 10^{-5}$ & $0.1^{\circ}$ & $75^{\circ}$ \\
\hline $04181+2654 \mathrm{~A}$ & 0.7 & $2.0 \times 10^{-14}$ & 50 & 5 & 10000 & TSC & 0.2 & $7.0 \times 10^{-5}$ & $7^{\circ}$ & $20^{\circ}$ \\
\hline $04239+2436$ & 1.5 & $1.3 \times 10^{-14}$ & 10 & 1 & 5000 & TSC & 0.1 & $1.0 \times 10^{-4}$ & $5^{\circ}$ & $15^{\circ}$ \\
\hline $04248+2612$ & 0.4 & $4.0 \times 10^{-15}$ & 30 & 5 & 10000 & TSC & 0.3 & $5.0 \times 10^{-5}$ & $15^{\circ}$ & $70^{\circ}$ \\
\hline $04264+2433$ & 0.7 & $5.0 \times 10^{-15}$ & 30 & 5 & 10000 & TSC & 0.8 & $5.0 \times 10^{-5}$ & $13^{\circ}$ & $87^{\circ}$ \\
\hline $04295+2251$ & 0.8 & $8.0 \times 10^{-15}$ & 20 & 5 & 10000 & TSC & 0.2 & $1.0 \times 10^{-4}$ & $5^{\circ}$ & $70^{\circ}$ \\
\hline $04302+2247$ & 1.0 & $3.0 \times 10^{-14}$ & 300 & 1 & 10000 & TSC & 0.3 & $4.0 \times 10^{-5}$ & $22^{\circ}$ & $89^{\circ}$ \\
\hline $04325+2402$ & 0.9 & $3.0 \times 10^{-14}$ & 100 & 3 & 5000 & 1.0 & 0.8 & $4.0 \times 10^{-5}$ & $15^{\circ}$ & $80^{\circ}$ \\
\hline $04361+2547$ & 4.0 & $2.0 \times 10^{-14}$ & 100 & 2 & 10000 & 1.5 & 0.9 & $7.0 \times 10^{-5}$ & $15^{\circ}$ & $80^{\circ}$ \\
\hline $04365+2535$ & 2.5 & $4.5 \times 10^{-14}$ & 50 & 1 & 10000 & TSC & 0.1 & $7.0 \times 10^{-5}$ & $5^{\circ}$ & $30^{\circ}$ \\
\hline $04368+2557$ & 1.8 & $4.0 \times 10^{-14}$ & 200 & 1 & 10000 & TSC & 0.5 & $1.0 \times 10^{-4}$ & $27^{\circ}$ & $89^{\circ}$ \\
\hline $04381+2540$ & 1.0 & $3.0 \times 10^{-14}$ & 70 & 5 & 10000 & TSC & 0.1 & $1.2 \times 10^{-4}$ & $10^{\circ}$ & $40^{\circ}$ \\
\hline $04489+3042$ & 0.3 & $1.0 \times 10^{-14}$ & 15 & 1 & 1000 & TSC & 0.7 & $4.0 \times 10^{-5}$ & $1^{\circ}$ & $20^{\circ}$ \\
\hline CoKu Tau/1 & 1.1 & $5.0 \times 10^{-15}$ & 40 & 7 & 5000 & TSC & 0.8 & $5.0 \times 10^{-5}$ & $5^{\circ}$ & $80^{\circ}$ \\
\hline DG Tau B & 2.5 & $3.5 \times 10^{-14}$ & 60 & 1 & 10000 & TSC & 0.1 & $3.0 \times 10^{-5}$ & $10^{\circ}$ & $55^{\circ}$ \\
\hline HL Tau & 8.0 & $4.5 \times 10^{-14}$ & 100 & 2 & 10000 & 1.0 & 0.2 & $4.0 \times 10^{-5}$ & $1^{\circ}$ & $15^{\circ}$ \\
\hline L1551 IRS5 & 25.0 & $7.0 \times 10^{-14}$ & 100 & 5 & 10000 & TSC & 0.3 & $3.0 \times 10^{-4}$ & $5^{\circ}$ & $45^{\circ}$ \\
\hline
\end{tabular}

Note. - Column (1) gives the name of the object, column (2) the total luminosity $\left(L_{s t a r}+L_{d i s k}\right)$ of the system, column (3) the reference density $\rho_{1}$, column (4) the centrifugal radius, column (5) the inner disk radius $\left(1 \mathrm{R}_{\text {star }}=2 \mathrm{R}_{\odot}\right)$, column (6) the outer radius of the envelope, column (7) gives the flattening parameter $\eta$, column (8) the fraction of the luminosity arising from the star, column (9) the $\mathrm{CO}_{2}$ ice abundance, column (10) the semi-opening angle of the cavity, and column (11) the inlincation angle.

Typical uncertainties for our model parameters are as follows: $L \pm 10 \%, \rho_{1} \pm 15 \%, R_{c} \pm 20 \%, \mathrm{R}_{\text {diskmin }} \pm 10 \%, \mathrm{R}_{\max } \pm 25 \%, \eta \pm 0.25$ (if not TSC model), $\eta_{\text {star }} \pm 0.05, \mathrm{CO}_{2}$ ice abundance $\pm 10 \%, \theta \pm 2^{\circ}, i \pm 5^{\circ}$. Note that most uncertainties are correlated; e.g., a decrease in $\rho_{1}$ should be accompanied by a decrease in $R_{c}$ and/or increase in $i$ to still result in a comparable fit. 
Spitzer Spectra and Models of Protostars in Taurus

TABLE 4

Comparison of Luminosities and InClination Angles

\begin{tabular}{|c|c|c|c|c|c|c|c|}
\hline $\begin{array}{c}\text { Name } \\
(1)\end{array}$ & $\begin{array}{c}\mathrm{L}_{b o l}\left(L_{\odot}\right) \\
\text { (literature) } \\
(2)\end{array}$ & $\begin{array}{l}\text { Ref. } \\
(3)\end{array}$ & $\begin{array}{c}\mathrm{L}_{b o l}\left(L_{\odot}\right) \\
(\text { measured }) \\
(4)\end{array}$ & $\begin{array}{c}\mathrm{L}_{b o l}\left(L_{\odot}\right) \\
(\text { model }) \\
(5)\end{array}$ & $\begin{array}{c}\mathrm{i}(\mathrm{deg}) \\
\text { (literature) } \\
(6)\end{array}$ & $\begin{array}{l}\text { Ref. } \\
(7)\end{array}$ & $\begin{array}{c}\mathrm{i}(\mathrm{deg}) \\
\text { (model) } \\
(8)\end{array}$ \\
\hline $04016+2610$ & 3.70 & 1 & 3.6 & 4.5 & 60 & 7 & 40 \\
\hline $04108+2803 \mathrm{~B}$ & 0.62 & 1 & 0.5 & 0.7 & $\cdots$ & . & 40 \\
\hline $04154+2823$ & 0.33 & 2 & 0.4 & 0.35 & $\ldots$ & $\ldots$ & 20 \\
\hline $04158+2805$ & 0.20 & 3 & 0.2 & 0.3 & $\ldots$ & $\ldots$ & 30 \\
\hline $04166+2706$ & $0.5(0.40)$ & $4(3)$ & 0.4 & 0.6 & $\ldots$ & $\ldots$ & 85 \\
\hline $04169+2702$ & $0.8(1.4)$ & $1(3)$ & 1.2 & 1.5 & 60 & 10 & 75 \\
\hline $04181+2654 \mathrm{~A}$ & $0.26(0.70)$ & $1(3)$ & 0.7 & 0.7 & $\cdots$ & ... & 20 \\
\hline $04181+2654 \mathrm{~B}$ & 0.25 & 1 & 0.3 & $\ldots$ & $\ldots$ & $\ldots$ & $\cdots$ \\
\hline $04239+2436(\mathrm{~A}, \mathrm{~B})$ & 1.27 & 1 & 1.5 & 1.5 & . & $\ldots$ & 15 \\
\hline $04248+2612(\mathrm{~A}, \mathrm{~B}, \mathrm{C})$ & 0.36 & 1 & 0.2 & 0.4 & 78 & 8 & 70 \\
\hline $04264+2433$ & 0.37 & 1 & 0.5 & 0.7 & $\cdots$ & $\cdots$ & 87 \\
\hline $04278+2253(\mathrm{~A}, \mathrm{~B})$ & 7.2 & 3 & 5.7 & $\ldots$ & $\ldots$ & $\ldots$ & $\ldots$ \\
\hline $04295+2251$ & $0.44(0.64)$ & $1(2)$ & 0.5 & 0.8 & $\ldots$ & $\ldots$ & 70 \\
\hline $04302+2247$ & 0.34 & 1 & 0.3 & 1.0 & 90 & 8 & 89 \\
\hline $04325+2402(\mathrm{~A}, \mathrm{~B}, \mathrm{C})$ & $0.9(0.70)$ & $5(1)$ & 1.2 & 0.9 & 60 & 7 & 80 \\
\hline $04361+2547(\mathrm{~A}, \mathrm{~B})$ & $3.8(2.90)$ & $3(1)$ & 2.5 & 4.0 & 60 & 7 & 80 \\
\hline $04365+2535$ & 2.4 & 2 & 2.8 & 2.5 & 55 & 7 & 30 \\
\hline $04368+2557(\mathrm{~A}, \mathrm{~B})$ & 1.6 & 3 & 1.9 & 1.8 & 75 & 7 & 89 \\
\hline $04381+2540(\mathrm{~A}, \mathrm{~B})$ & 0.73 & 2 & 0.7 & 1.0 & 55 & 7 & 40 \\
\hline $04489+3042$ & 0.30 & 1 & 0.3 & 0.3 & $\cdots$ & $\cdots$ & 20 \\
\hline CoKu Tau/1 (A,B) & $>0.29$ & 1 & 1.0 & 1.1 & $\sim 90$ & 9 & 80 \\
\hline DG Tau B & $>0.02$ & 1 & 1.8 & 2.5 & $\sim 90$ & 9 & 55 \\
\hline GV Tau (A,B) & 6.98 & 1 & 9.0 & $\ldots$ & 30 & 7 & $\ldots$ \\
\hline HН 30 & $>0.1$ & 6 & 0.02 & $\ldots$ & $\sim 90$ & 12 & $\ldots$ \\
\hline HL Tau & $6.60(7.1)$ & $1(6)$ & 6.9 & 8.0 & 67 & 11 & 15 \\
\hline IC 2087 IR & 3.80 & 1 & 4.9 & $\cdots$ & & $\cdots$ & $\cdots$ \\
\hline L1551 IRS5 (A,B) & $28(21.90)$ & $5(1)$ & 23.2 & 25.0 & $65(56)$ & $7(8)$ & 45 \\
\hline LkHa 358 & 0.59 & 13 & 0.3 & & & & \\
\hline
\end{tabular}

References. - (1) Kenyon \& Hartmann (1995); (2) Myers et al. (1987); (3) Kenyon et al. (1990) (4) Young et al. (2003); (5) Motte \& André (2001); (6) Reipurth et al. (1993); (7) Hogerheijde et al. (1998); (8) Lucas \& Roche (1997); (9) Padgett et al. (1999); (10) Ohashi et al. (1997b); (11) Close et al. (1997); (12) Burrows et al. (1996); (13) Luhman (2000)

Note. - Column (1) gives the name of the object, columns (2) and (3) the bolometric luminosity of the source taken from the literature and its reference, respectively, column (4) gives the bolometric luminosity measured by integrating under the SED, column (5) the luminosity used in the models, columns (6) and (7) give the inclination angle taken from the literature and its reference, respectively, and column (8) gives the inclination angle used in the models. 


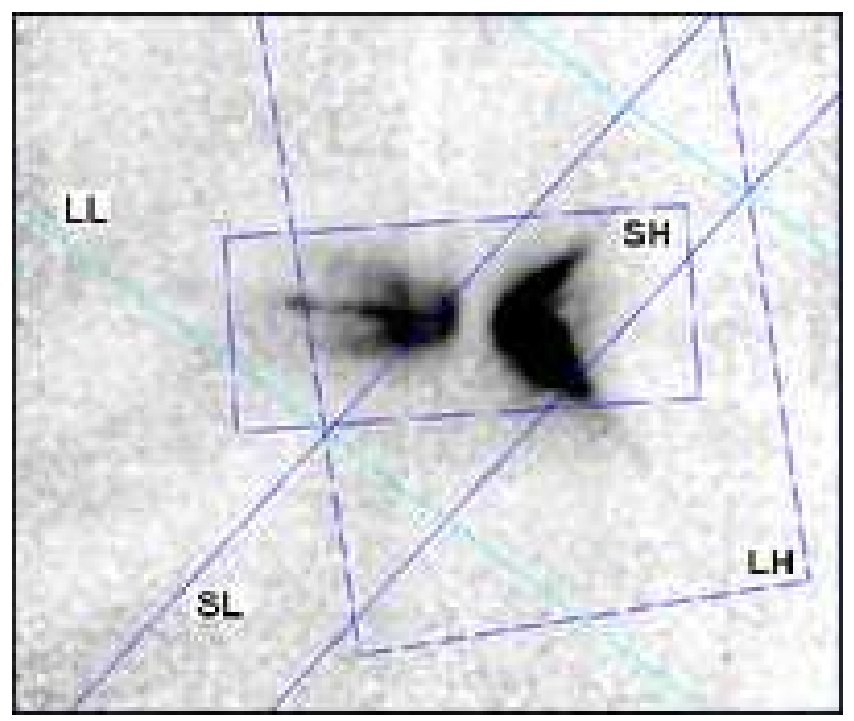

FIG. 1.- The IRS SL, LL, SH, and LH slit positions (only one of the two nod positions is shown) superposed on the NICMOS J-band (F110W) image of DG Tau B from Padgett et al. (1999); note that for this object the LL module was not used and is shown for illustrative purposes only. The slits, with their different widths and orientations, cover different parts of the object.

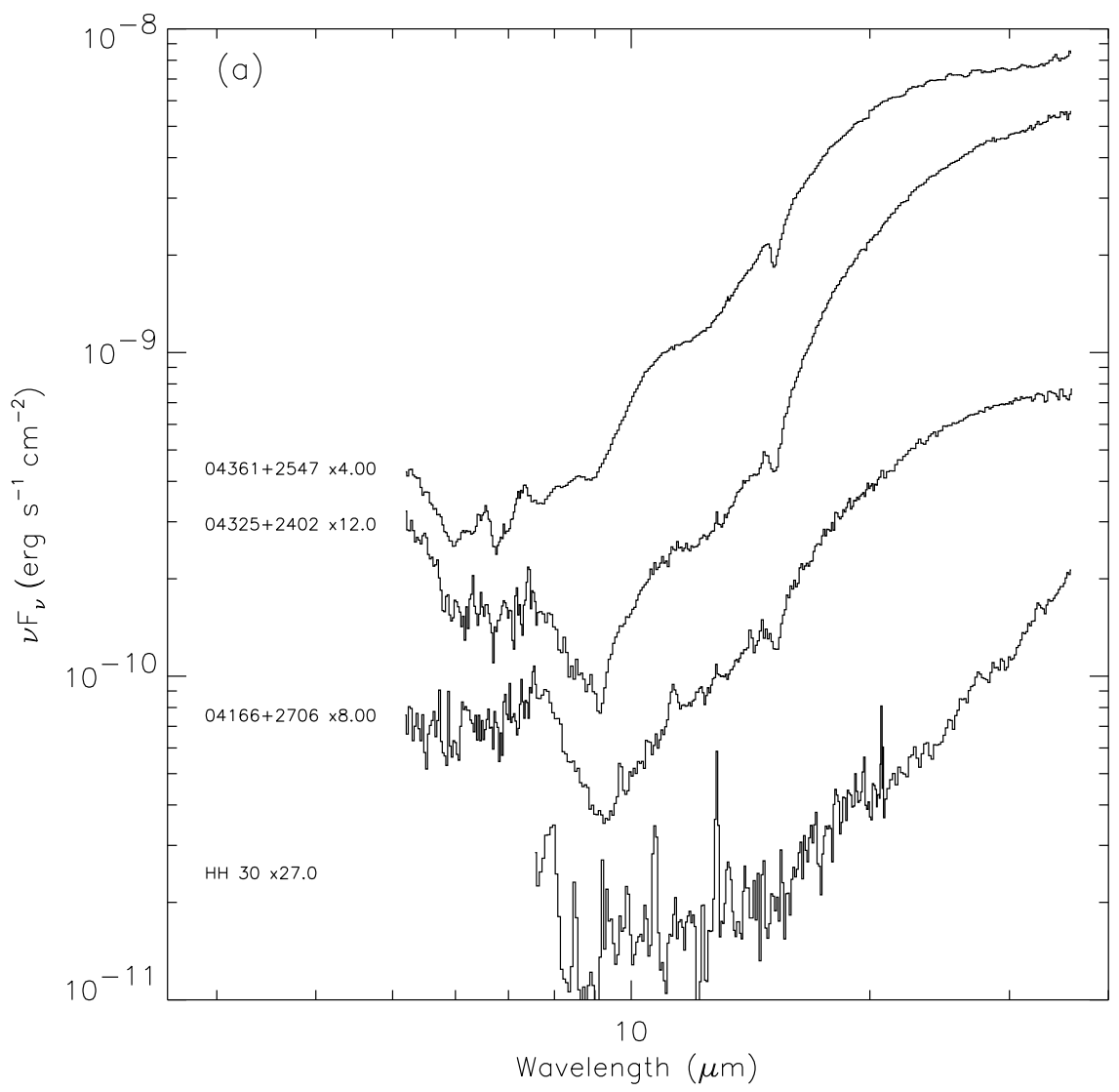

FIG. 2.- IRS Spectra of Class I Objects in Taurus. 


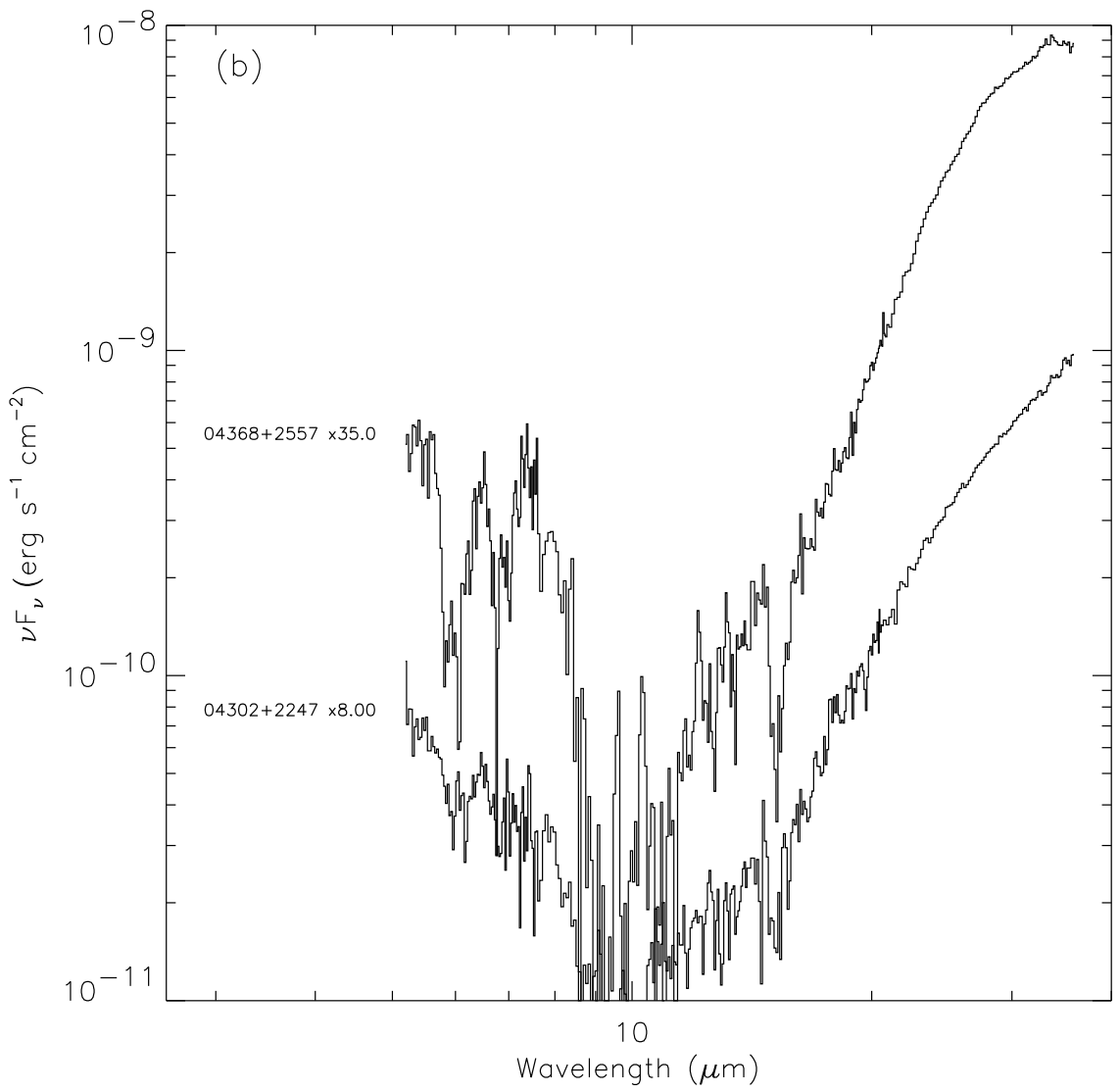

Fig. 2. - continued.

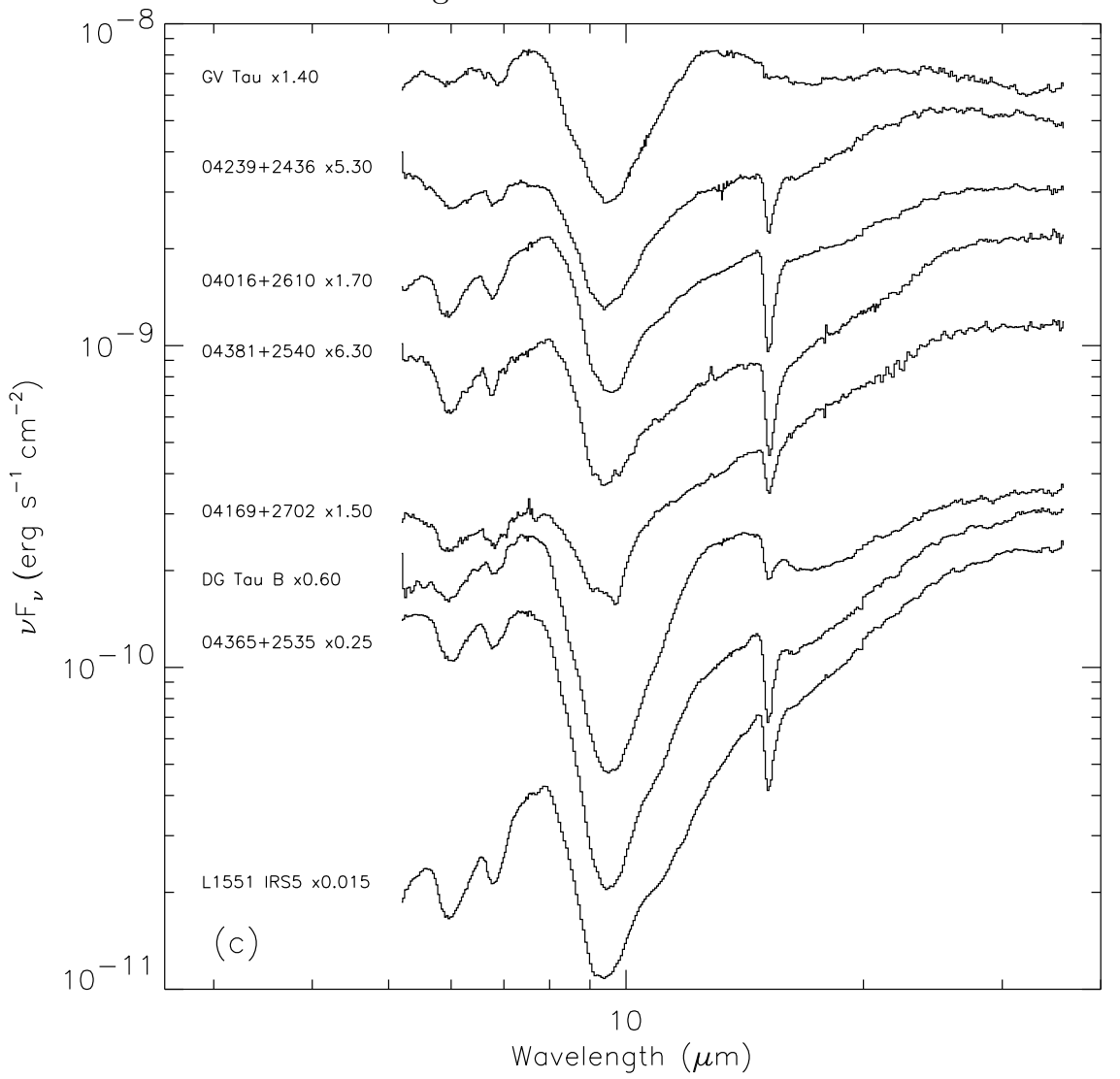

Fig. 2. - continued. 
Furlan et al.

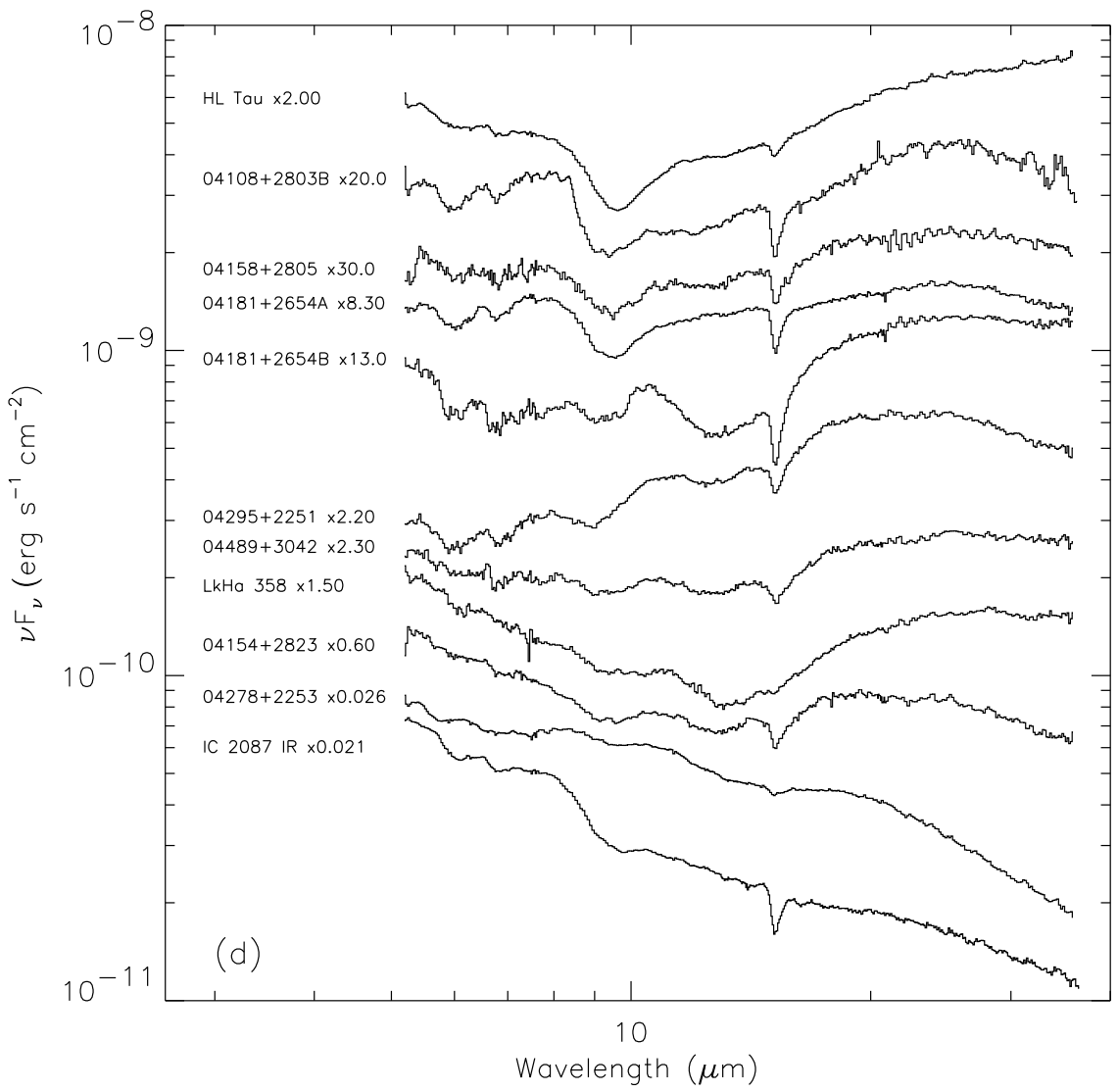

Fig. 2. - continued.

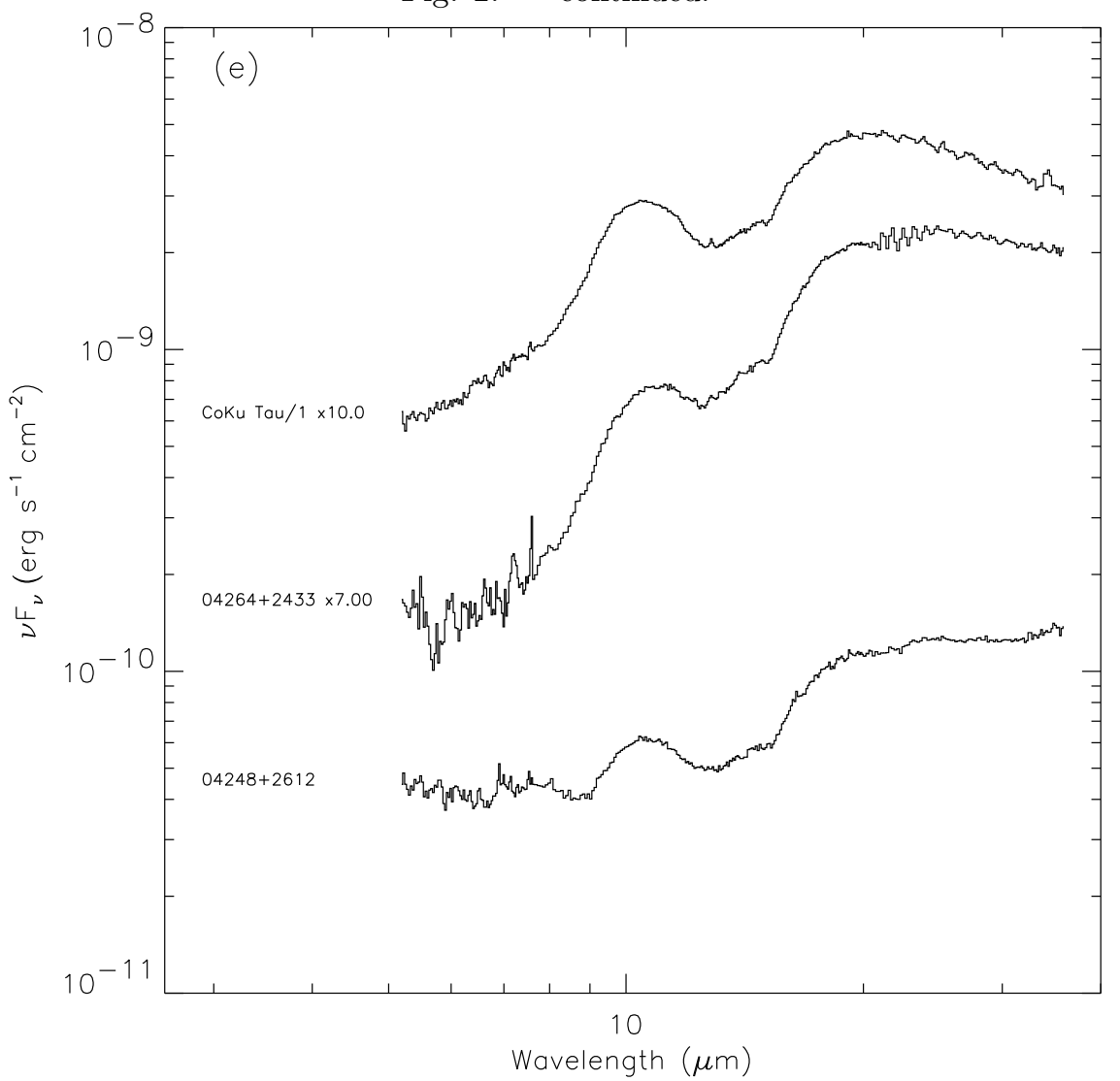

Fig. 2. - continued. 


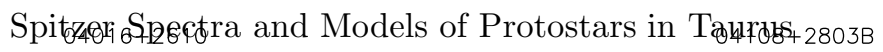
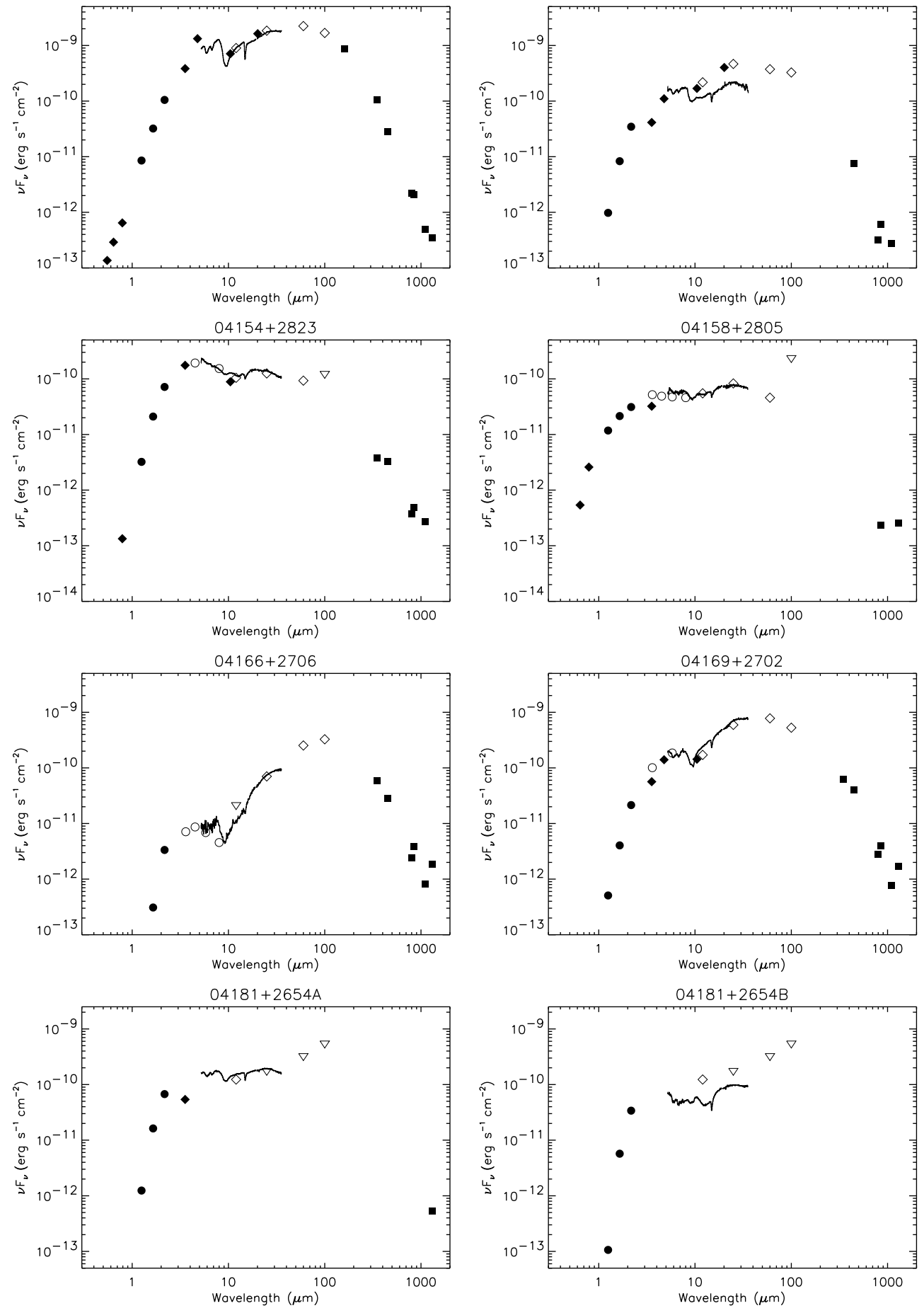

FIG. 3.- SED plots of the Class I objects in our sample, ordered alphabetically by their name. Optical to mid-IR, ground-based photometry is shown as filled diamonds, the 2MASS J, $\mathrm{H}$, and $\mathrm{K}_{s}$ fluxes as filled circles, the IRAC 3.6, 4.5, 5.8, and 8.0 $\mu$ m fluxes as open circles, the IRAS 12, 25, and $60 \mu \mathrm{m}$ fluxes as open diamonds or open, upside down triangles, if upper limit, and sub-mm and mm fluxes, where available, as filled squares. The IRS spectrum is also shown. No corrections for reddening have been applied. 
Furlan et al.
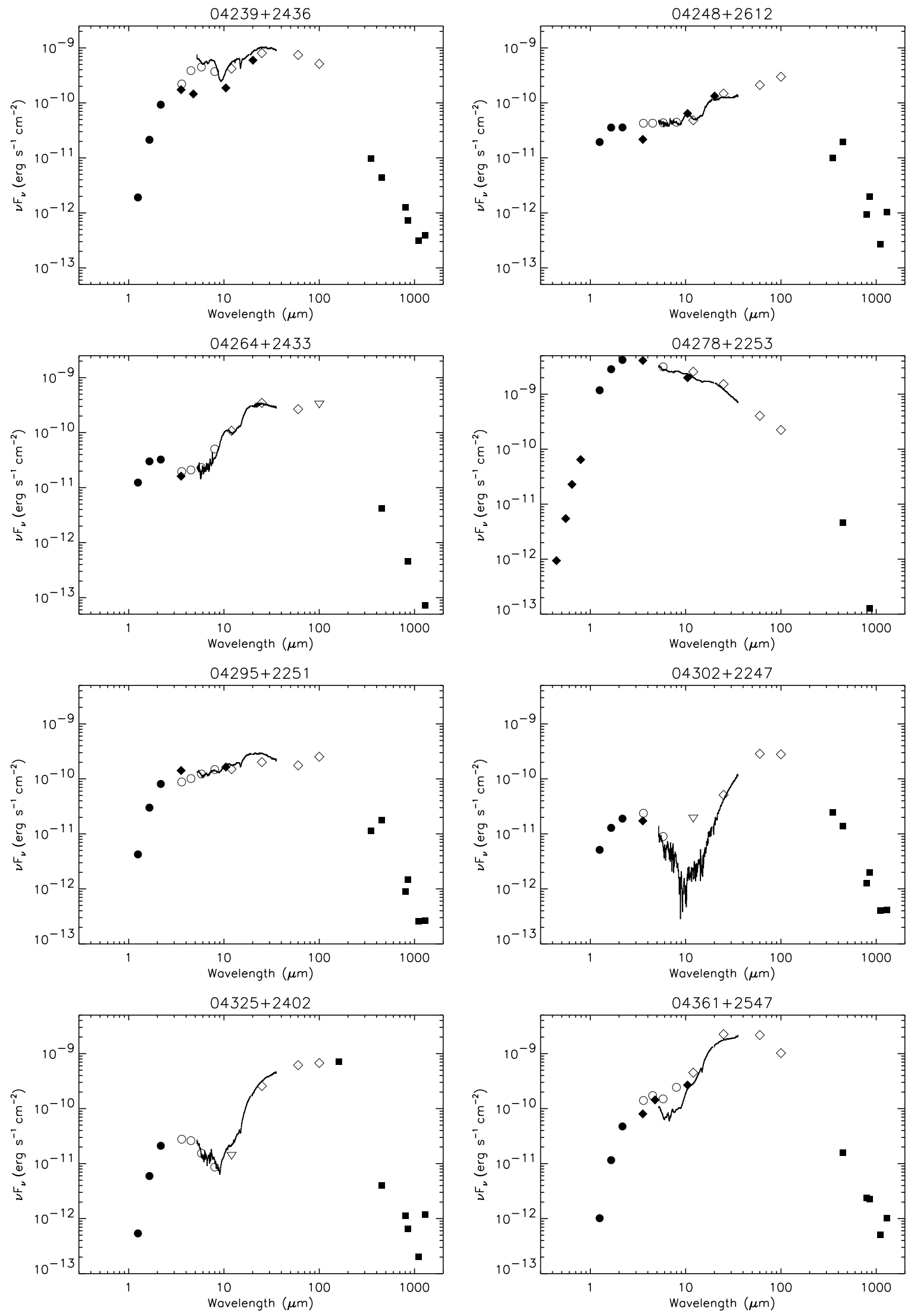

Fig. 3. - continued. 
Spitzer Spectra and Models of Protostars in Taurus
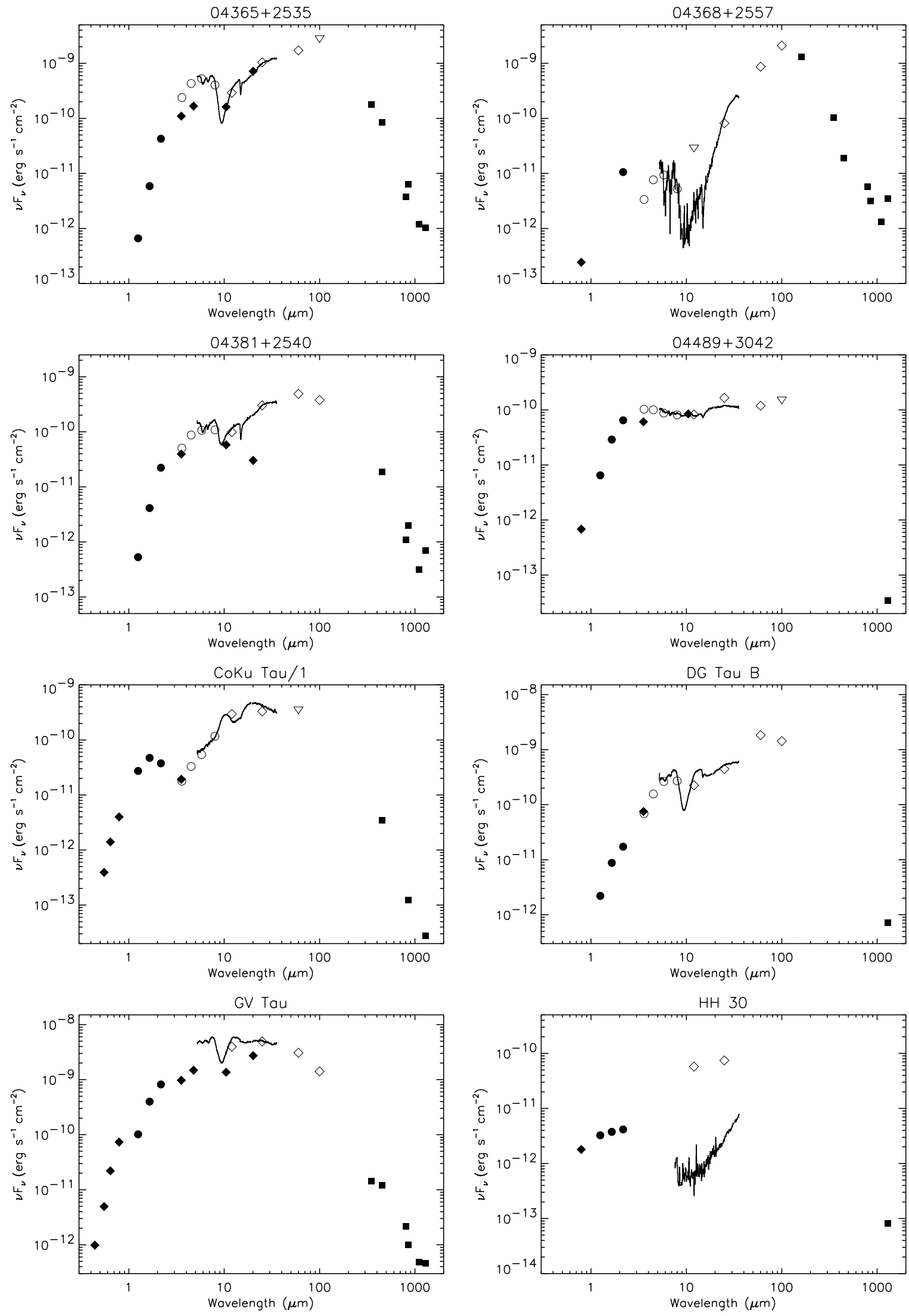

Fig. 3. - continued. 
Furlan et al.
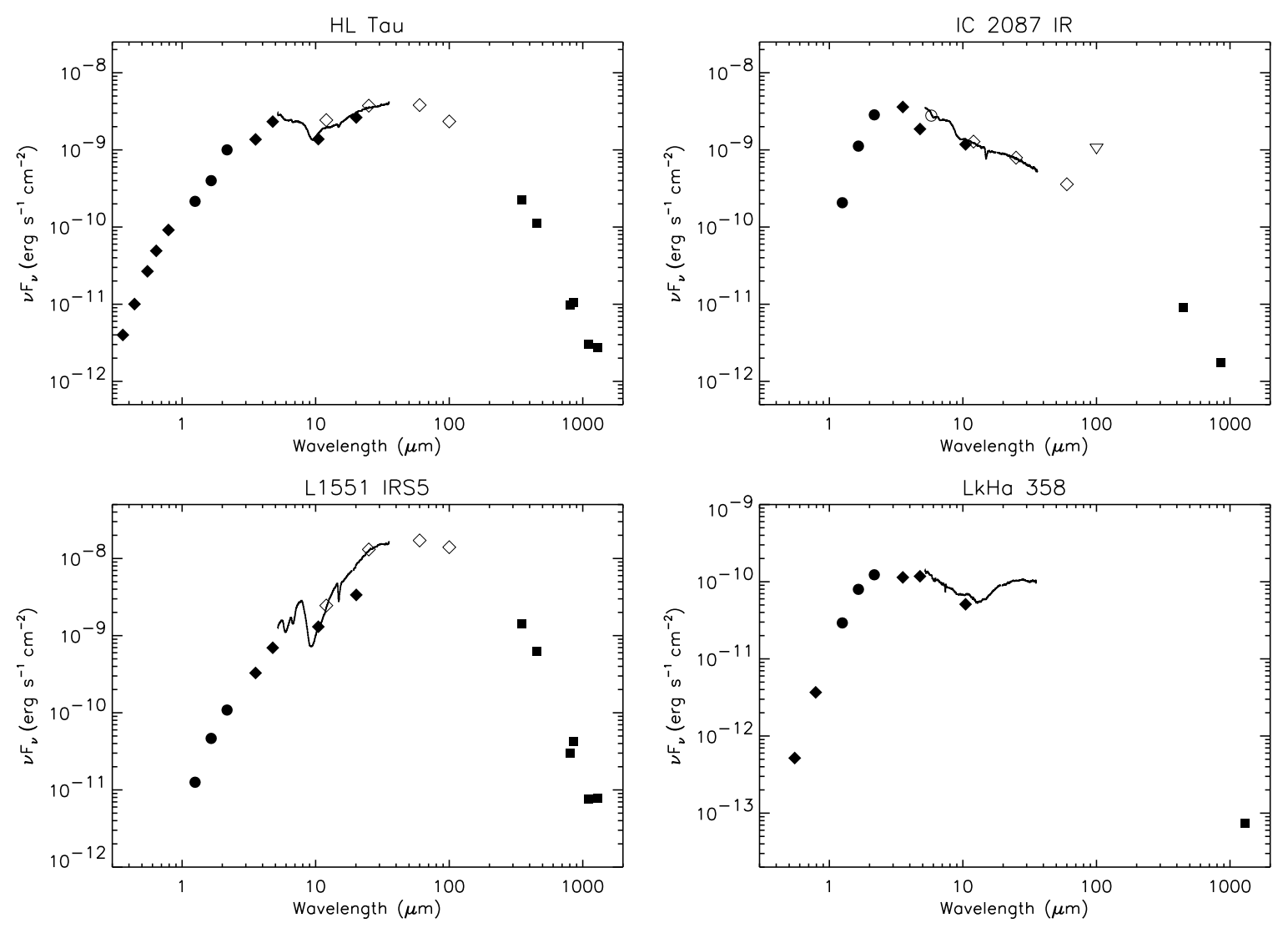

Fig. 3. - continued.
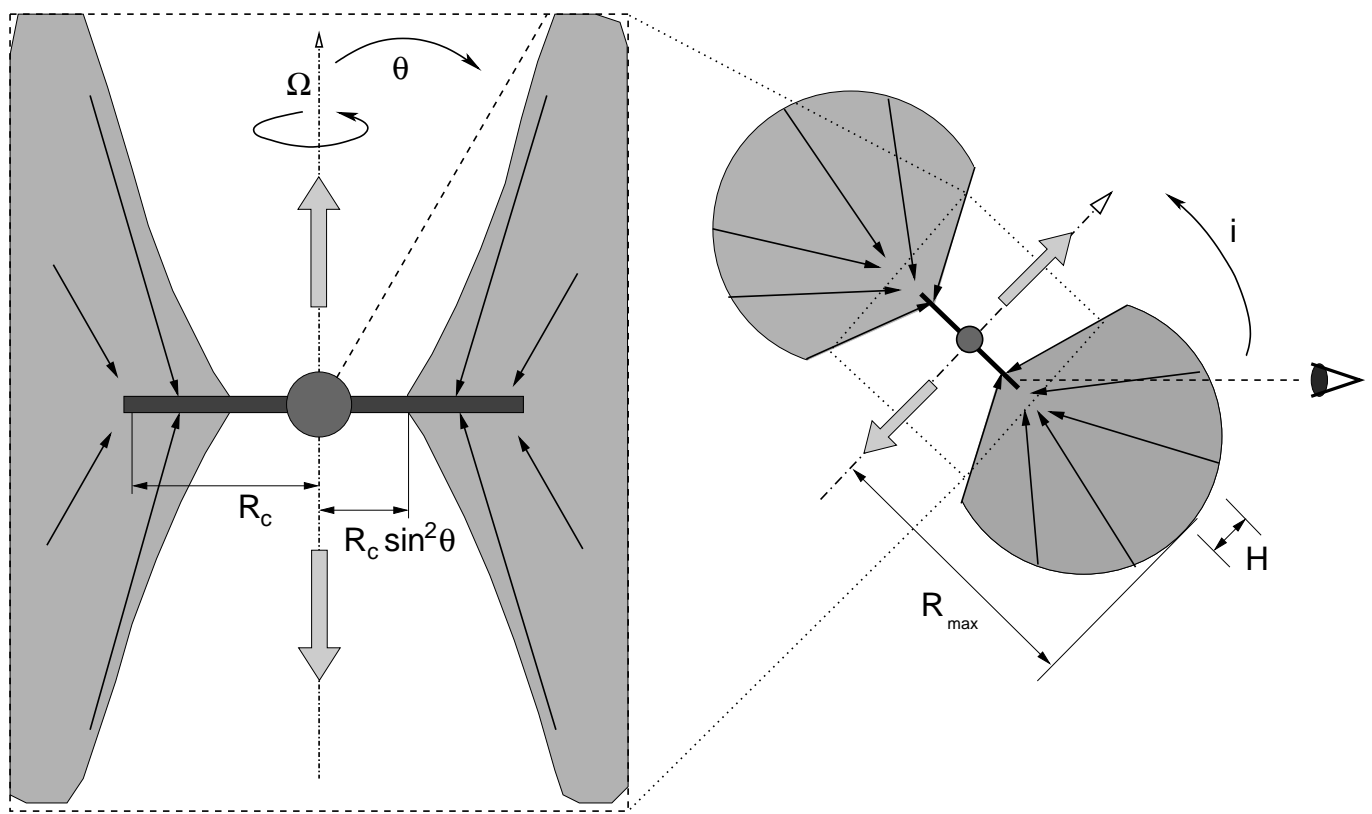

FIG. 4.- A sketch of an envelope model as described in $\S 4.1$ 


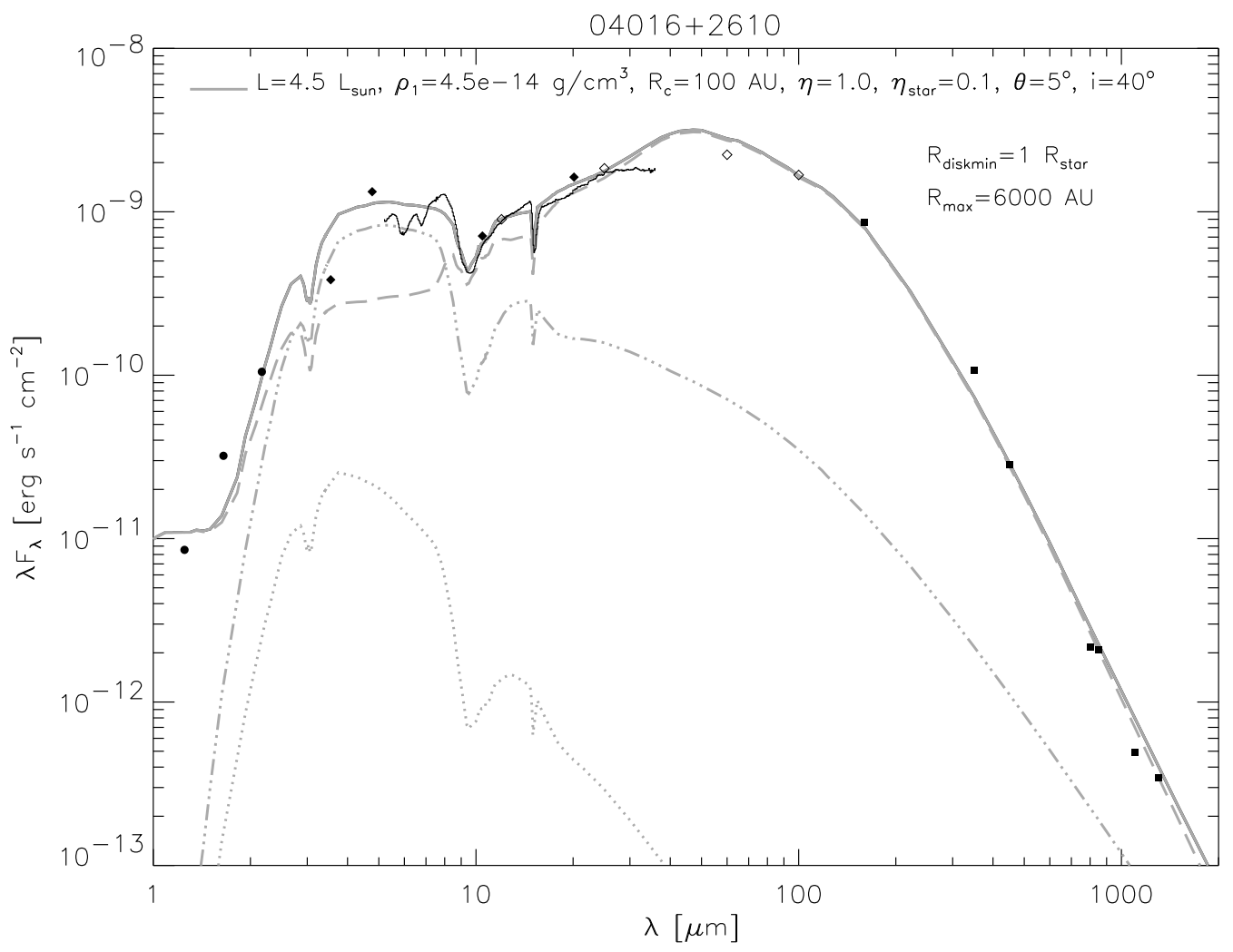

FIG. 5. - IRS spectrum and photometric data of $04016+2610$, and an envelope model fit with $\mathrm{L}=4.5 \mathrm{~L} \odot, \rho_{1}=4.5 \times 10^{-14} \mathrm{~g} \mathrm{~cm}^{-3}$, $R_{c}=100 \mathrm{AU}, \eta=1.0, \eta_{\text {star }}=0.1, \theta=5^{\circ}, \mathrm{i}=40^{\circ}$, an inner disk radius of 1 stellar radius, and an outer envelope radius of $6000 \mathrm{AU}$. The $\mathrm{CO} \mathrm{C}_{2}$ ice abundance set to $1.5 \times 10^{-4}$. The gray lines represent the different model components: the envelope (long-dashed line), the star (dotted line) and the disk (dash-dotted line), both extinguished by the envelope, and the sum of all components (solid gray line).

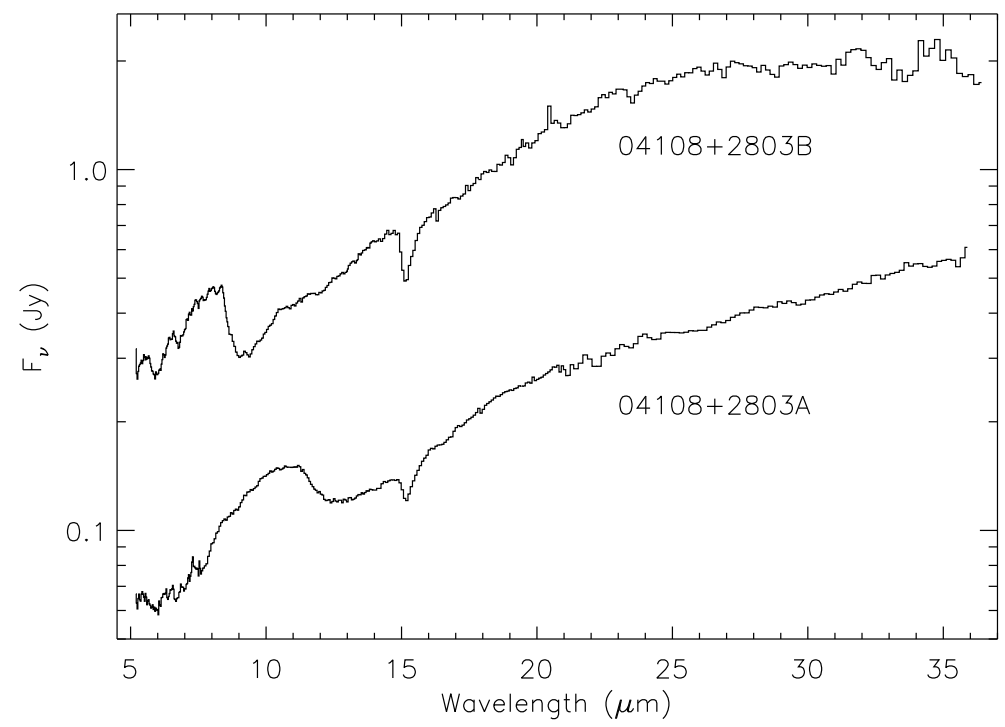

FIG. 6. - IRS spectra of both components of the $21^{\prime \prime}$ binary IRAS $04108+2803$, plotted on the same scale. While observing $04108+2803 \mathrm{~A}$, the bright B component partly entered the LL slit, and therefore the spectrum of component A beyond $14 \mu \mathrm{m}$ contains an increasing amount of emission from component B; the spectrum of A is dominated by emission from B beyond about $20 \mu \mathrm{m}$. 


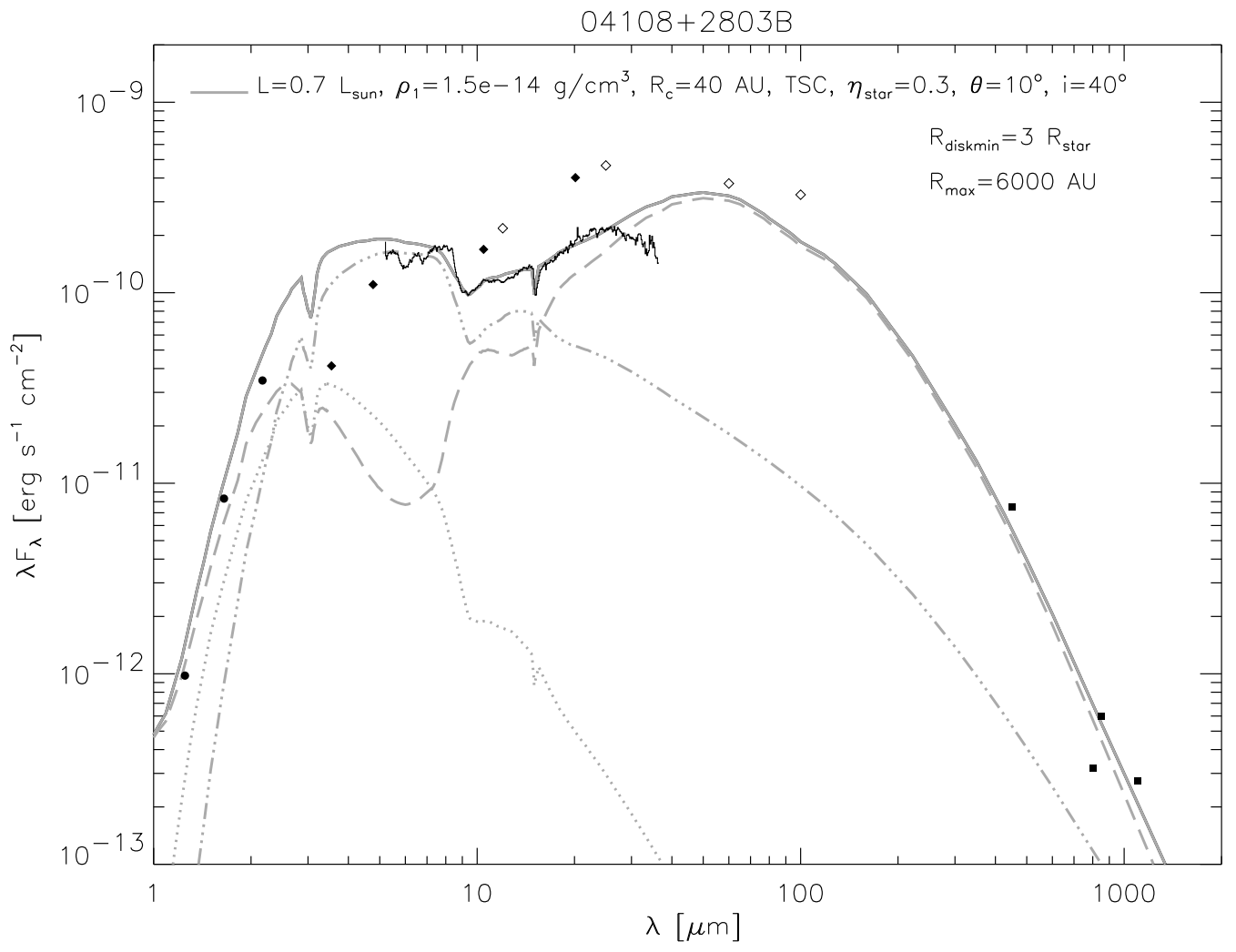

FIG. 7.- IRS spectrum and photometric data of $04108+2803 \mathrm{~B}$, and an envelope model fit with $\mathrm{L}=0.7 \mathrm{~L}_{\odot}, \rho_{1}=1.5 \times 10^{-14} \mathrm{~g} \mathrm{~cm}^{-3}$, $R_{c}=40 \mathrm{AU}$, initial TSC density distribution, $\eta_{\text {star }}=0.3, \theta=10^{\circ}, \mathrm{i}=40^{\circ}$, an inner disk radius of 3 stellar radii, and an outer envelope radius of $6000 \mathrm{AU}$. The $\mathrm{CO}_{2}$ ice abundance was set to $1.0 \times 10^{-4}$. The gray lines represent the model components as in Figure 5

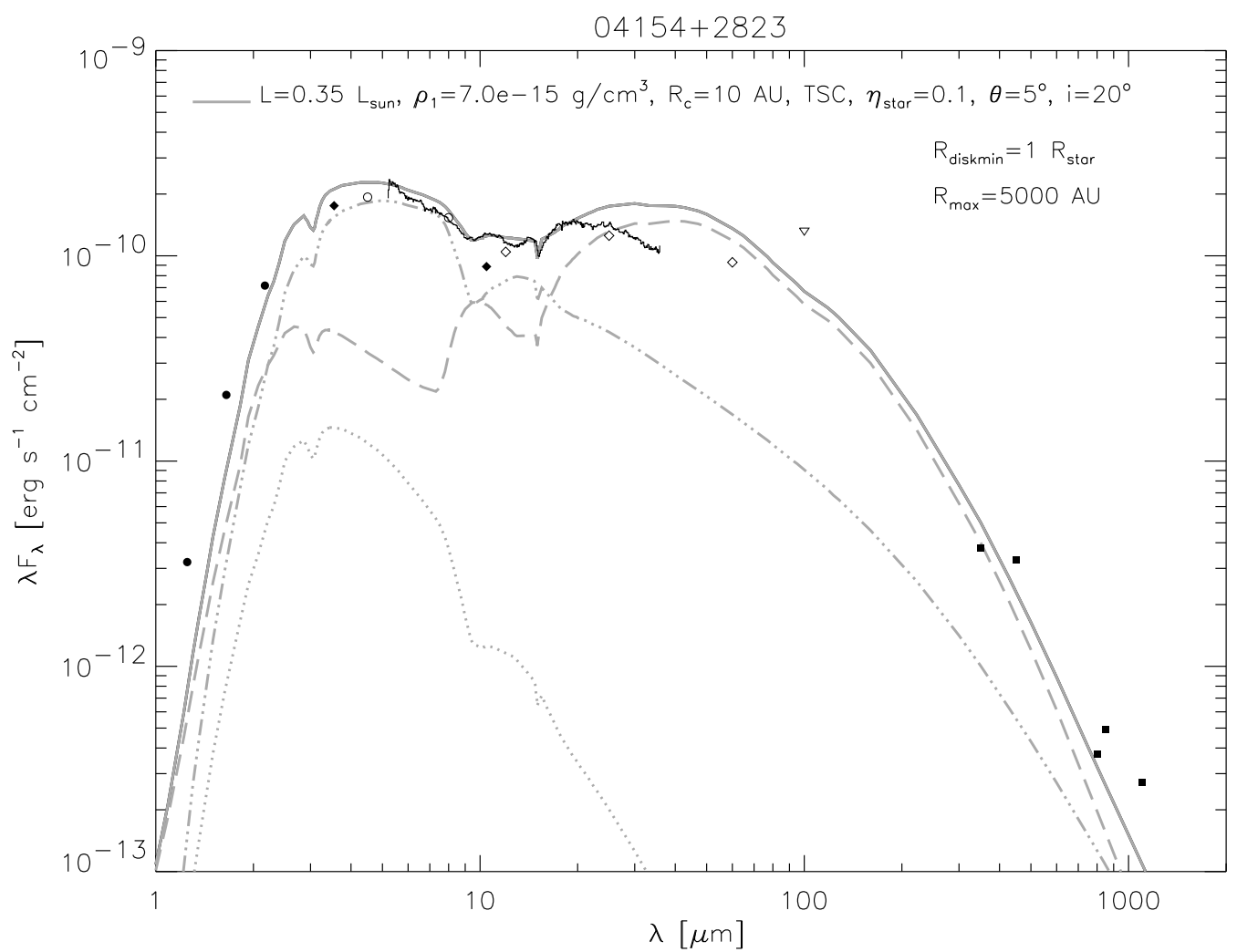

FIG. 8. - IRS spectrum and photometric data of $04154+2823$, and an envelope model fit with $\mathrm{L}=0.35 \mathrm{~L}_{\odot}, \rho_{1}=7.0 \times 10^{-15} \mathrm{~g} \mathrm{~cm}^{-3}$, $R_{c}=10 \mathrm{AU}$, initial TSC density distribution, $\eta_{\text {star }}=0.1, \theta=5^{\circ}, \mathrm{i}=20^{\circ}$, an inner disk radius of 1 stellar radius, and an outer envelope radius of $5000 \mathrm{AU}$. The $\mathrm{CO}_{2}$ ice abundance was set to $1.0 \times 10^{-4}$. The gray lines represent the model components as in Figure 5 


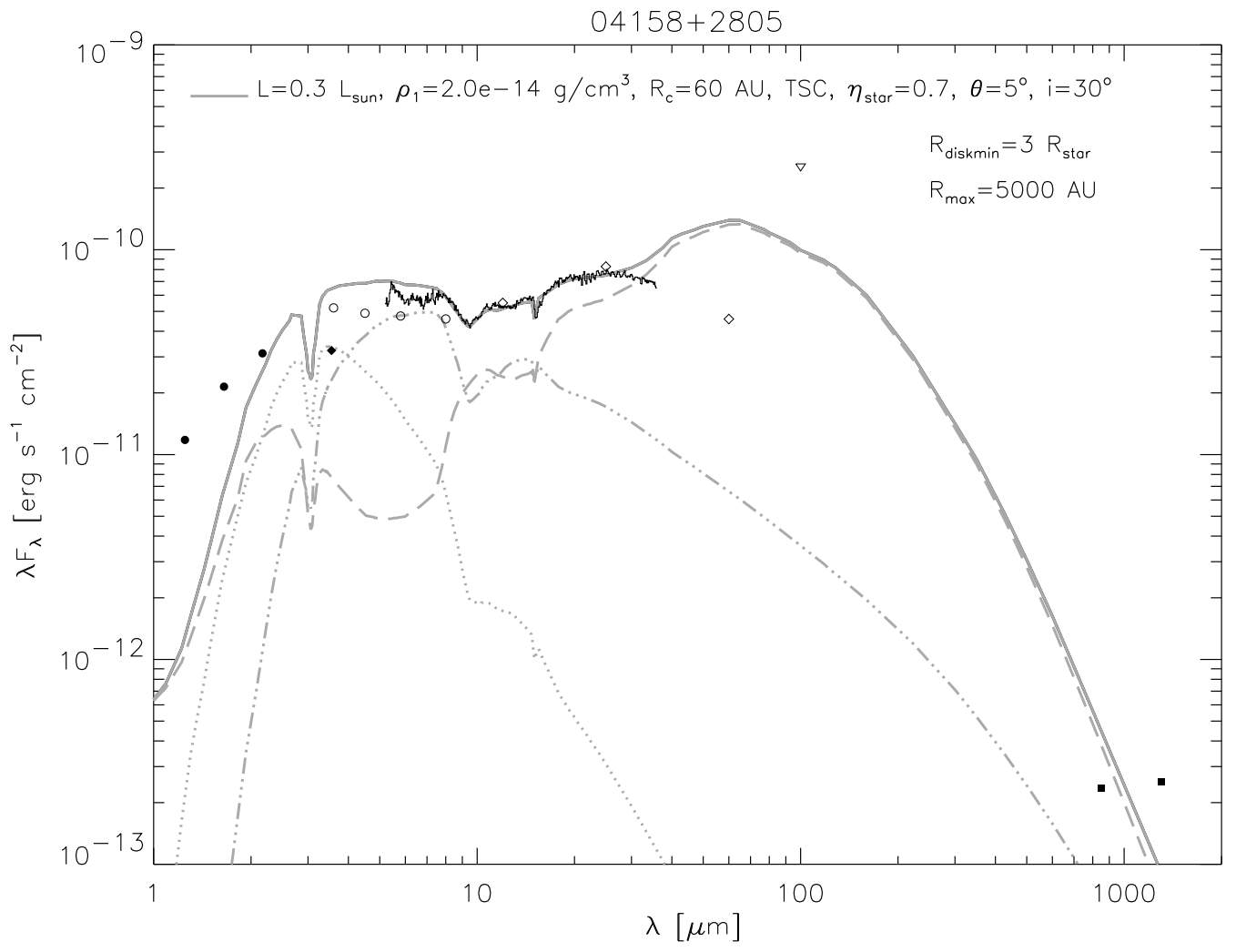

FIG. 9.- IRS spectrum and photometric data of $04158+2805$, and an envelope model fit with $\mathrm{L}=0.3 \mathrm{~L} \odot, \rho_{1}=2.0 \times 10^{-14} \mathrm{~g} \mathrm{~cm}^{-3}$, $R_{c}=60 \mathrm{AU}$, initial TSC density distribution, $\eta_{s t a r}=0.7, \theta=5^{\circ}, \mathrm{i}=30^{\circ}$, an inner disk radius of 3 stellar radii, and an outer envelope radius of $5000 \mathrm{AU}$. The $\mathrm{CO}_{2}$ ice abundance was set to $4.0 \times 10^{-5}$. The gray lines represent the model components as in Figure 5

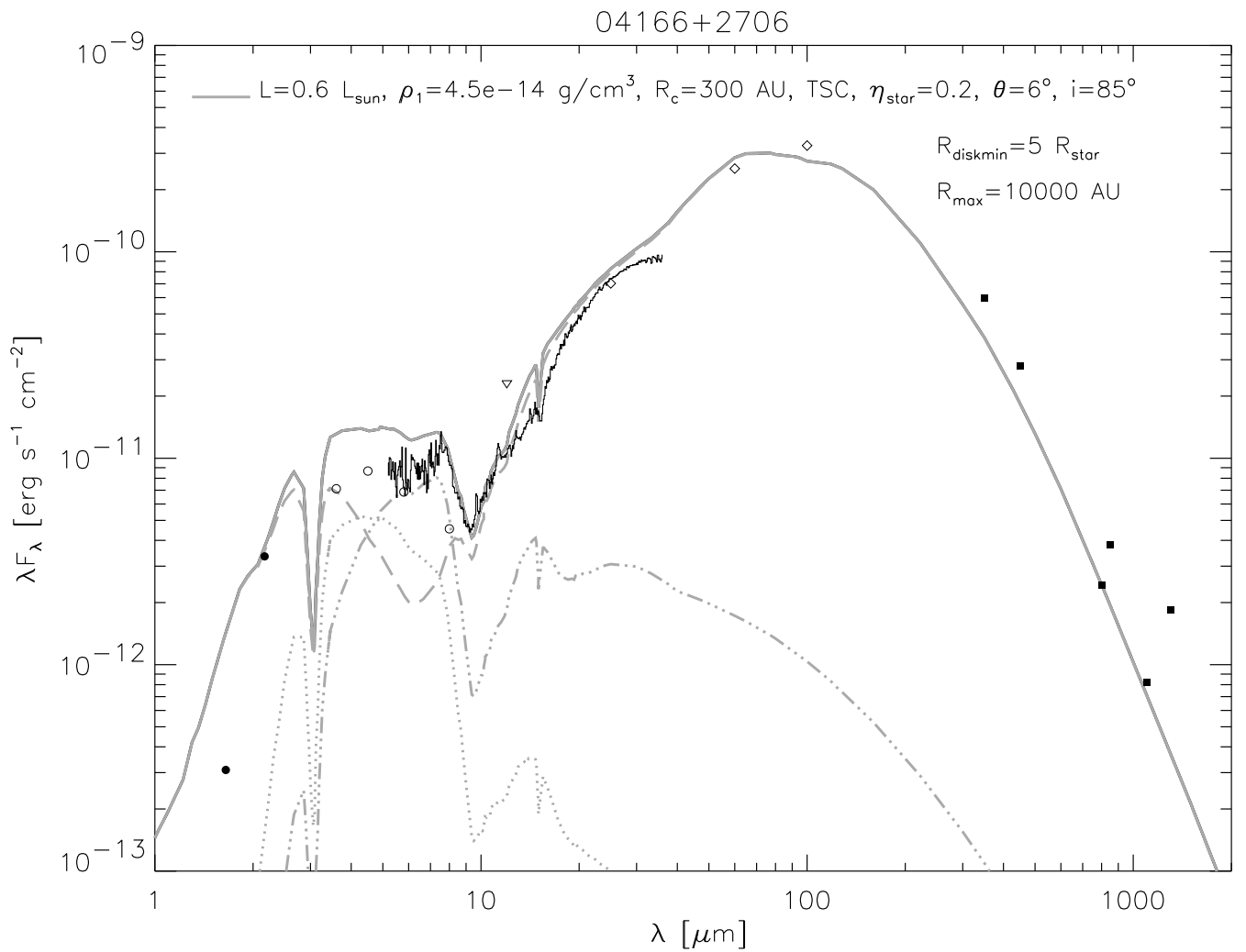

FIG. 10.- IRS spectrum and photometric data of 04166+2706, and an envelope model fit with $\mathrm{L}=0.6 \mathrm{~L} \odot, \rho_{1}=4.5 \times 10^{-14} \mathrm{~g} \mathrm{~cm}^{-3}$, $R_{c}=300 \mathrm{AU}$, initial TSC density distribution, $\eta_{\text {star }}=0.2, \theta=6^{\circ}, \mathrm{i}=85^{\circ}$, an inner disk radius of 5 stellar radii, and an outer envelope radius of $10000 \mathrm{AU}$. The $\mathrm{CO}_{2}$ ice abundance was set to $2.0 \times 10^{-5}$. The gray lines represent the model components as in Figure 5 


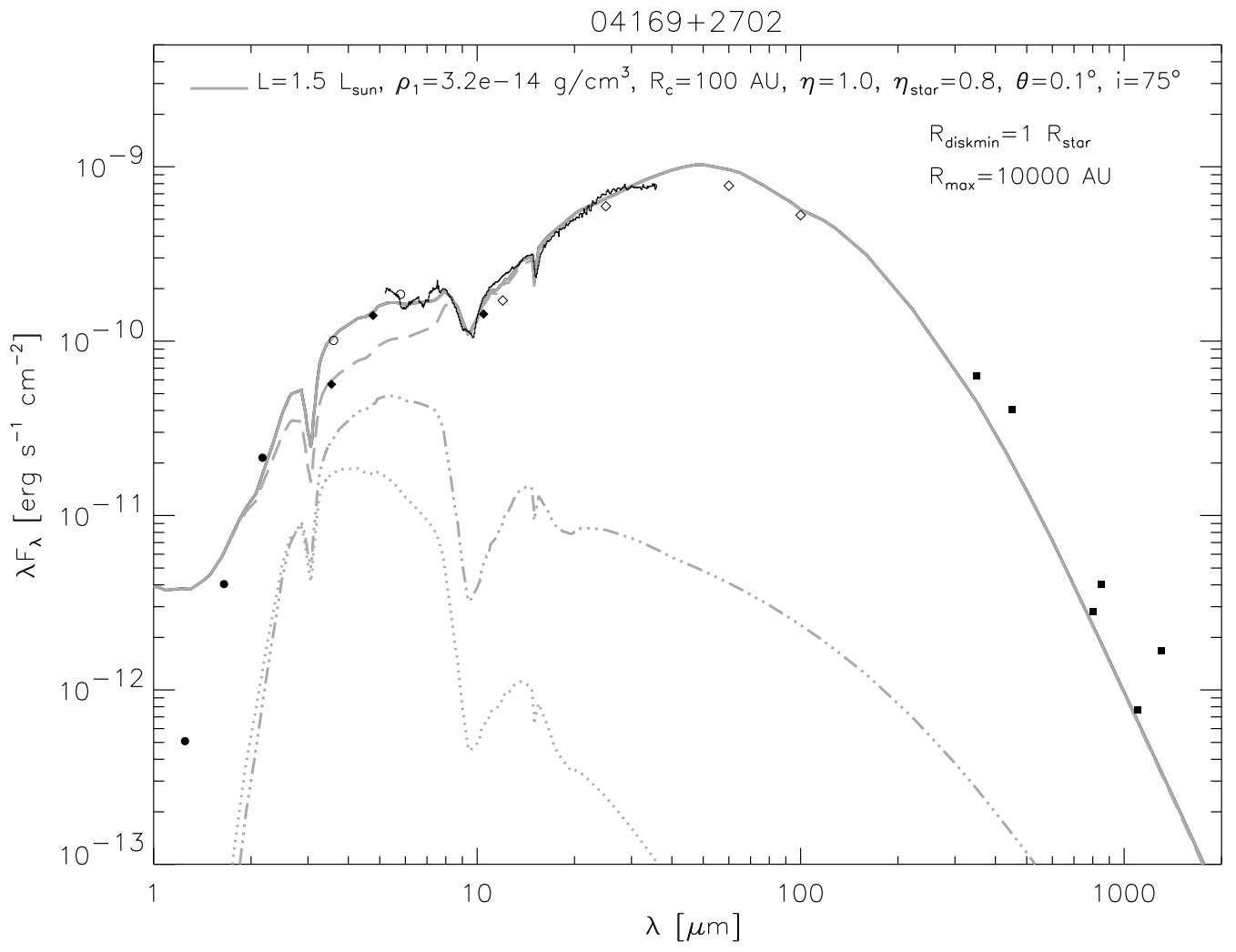

FIG. 11.- IRS spectrum and photometric data of 04169+2702, and an envelope model fit with $\mathrm{L}=1.5 \mathrm{~L}_{\odot}, \rho_{1}=3.2 \times 10^{-14} \mathrm{~g} \mathrm{~cm}^{-3}$, $R_{c}=100 \mathrm{AU}, \eta=1.0, \eta_{\text {star }}=0.8, \theta=0.1^{\circ}, \mathrm{i}=75^{\circ}$, an inner disk radius of 1 stellar radius, and an outer envelope radius of $10000 \mathrm{AU}$. The $\mathrm{CO}_{2}$ ice abundance was set to $6.0 \times 10^{-5}$. The gray lines represent the model components as in Figure 5

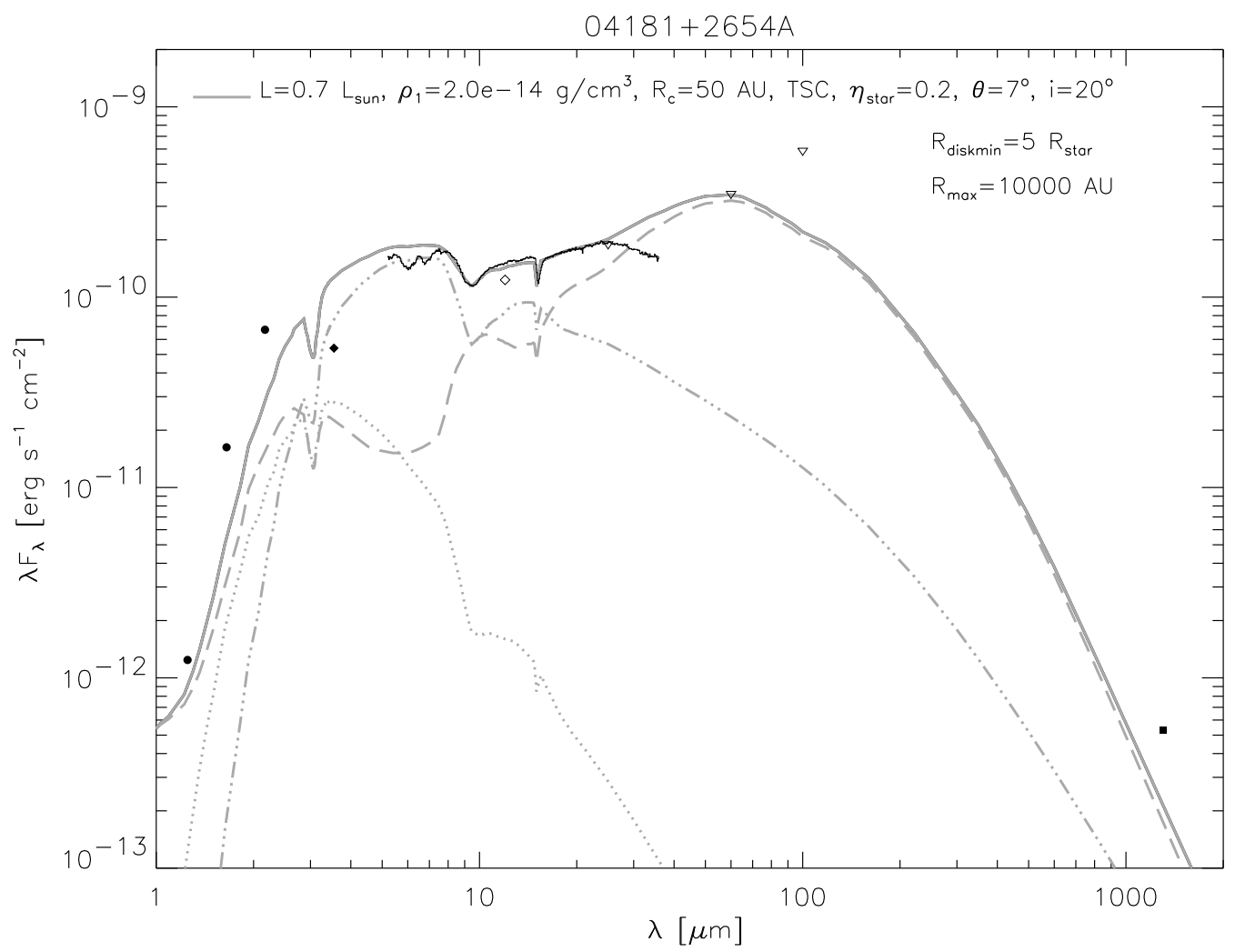

FIG. 12.- IRS spectrum and photometric data of $04181+2654 \mathrm{~A}$, and an envelope model fit with $\mathrm{L}=0.7 \mathrm{~L}_{\odot}, \rho_{1}=2.0 \times 10^{-14} \mathrm{~g} \mathrm{~cm}^{-3}$, $R_{c}=50 \mathrm{AU}$, initial TSC density distribution, $\eta_{\text {star }}=0.2, \theta=7^{\circ}, \mathrm{i}=20^{\circ}$, an inner disk radius of 5 stellar radii, and an outer envelope radius of $10000 \mathrm{AU}$. The $\mathrm{CO}_{2}$ ice abundance was set to $7.0 \times 10^{-5}$. The gray lines represent the model components as in Figure 5 


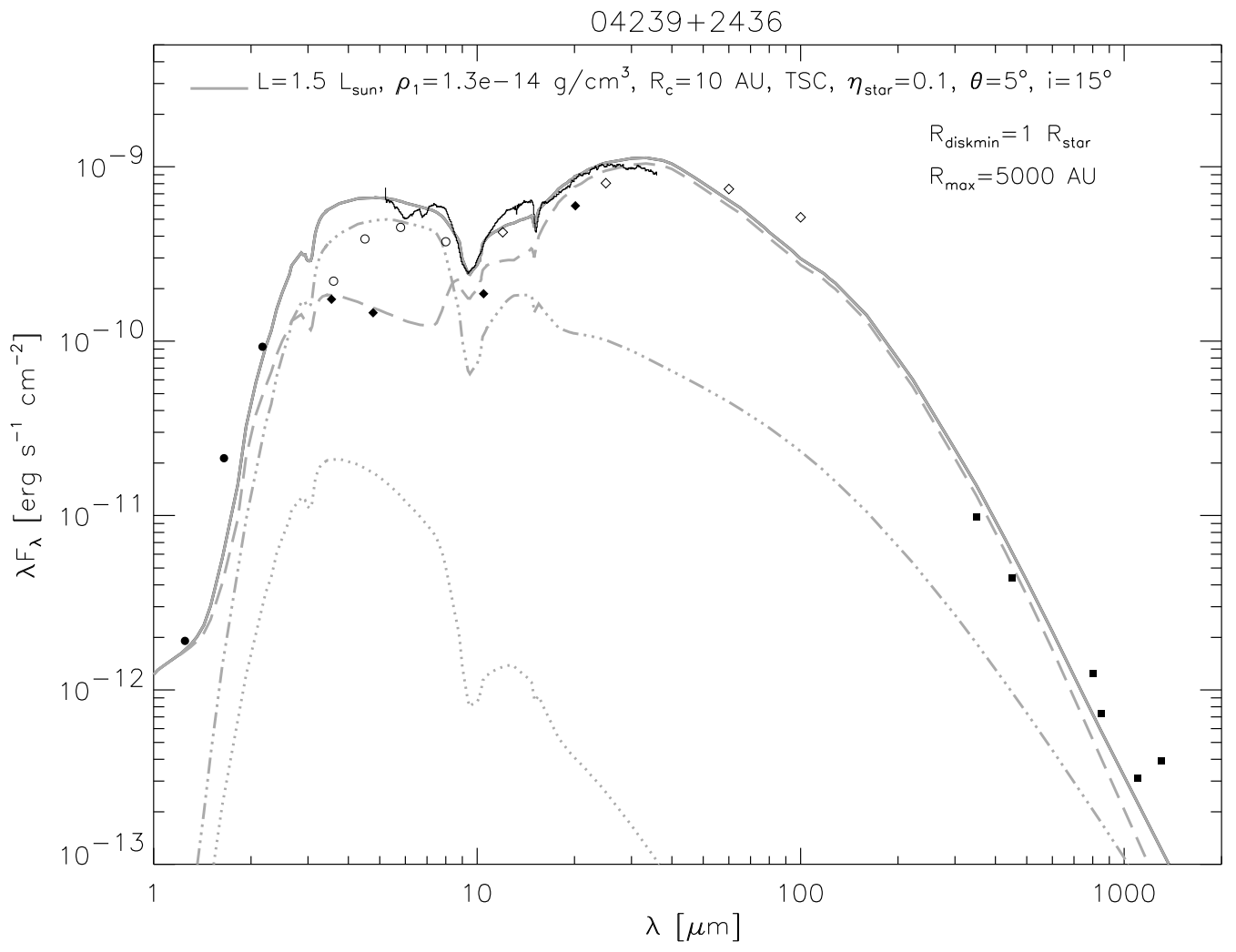

FIG. 13.- IRS spectrum and photometric data of 04239+2436, and an envelope model fit with $\mathrm{L}=1.5 \mathrm{~L}_{\odot}, \rho_{1}=1.3 \times 10^{-14} \mathrm{~g} \mathrm{~cm}^{-3}$, $R_{c}=10 \mathrm{AU}$, initial TSC density distribution, $\eta_{\text {star }}=0.1, \theta=5^{\circ}, \mathrm{i}=15^{\circ}$, an inner disk radius of 1 stellar radius, and an outer envelope radius of $5000 \mathrm{AU}$. The $\mathrm{CO}_{2}$ ice abundance was set to $1.0 \times 10^{-4}$. The gray lines represent the model components as in Figure 5

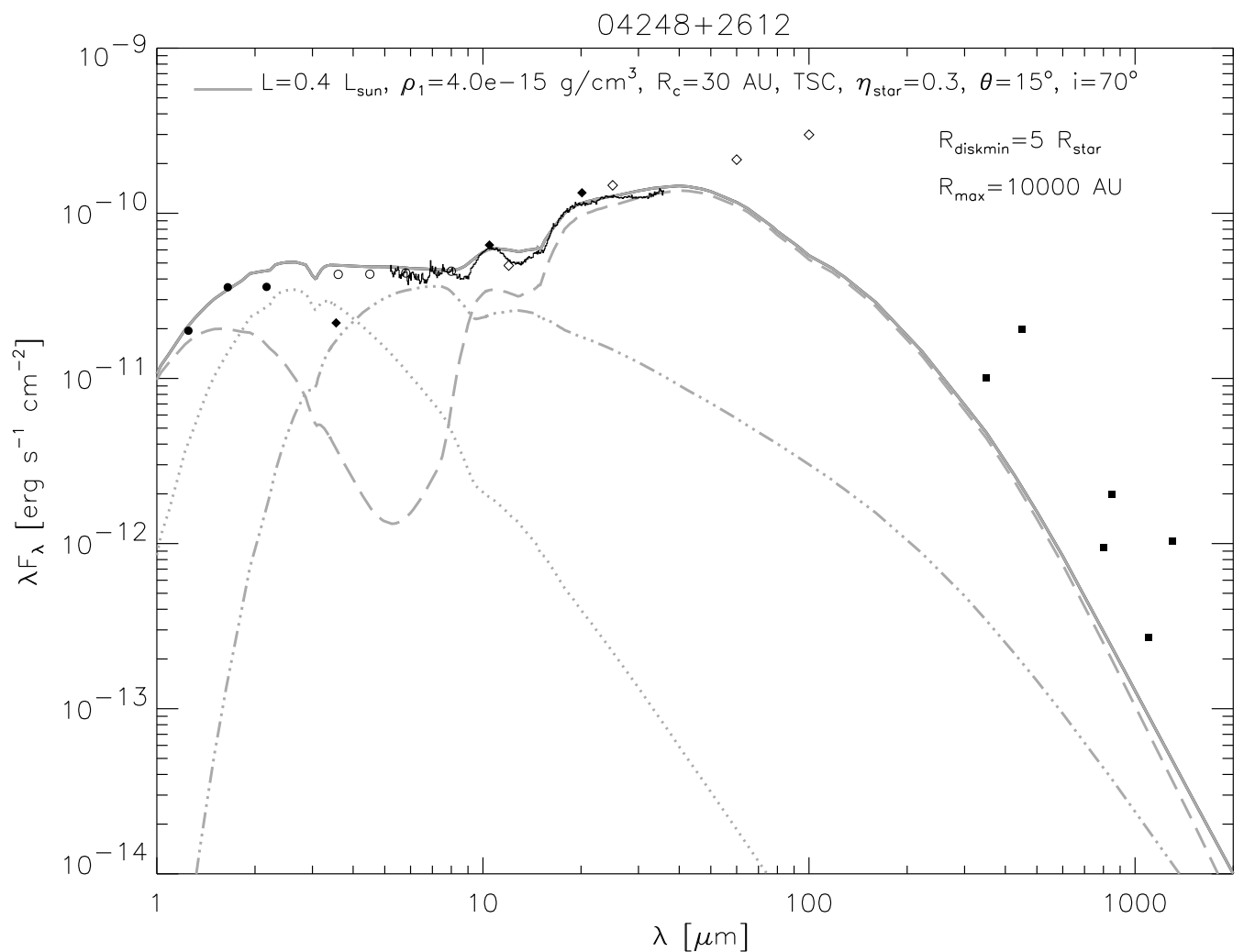

FIG. 14.- IRS spectrum and photometric data of 04248+2612, and an envelope model fit with $\mathrm{L}=0.4 \mathrm{~L}_{\odot}, \rho_{1}=4.0 \times 10^{-15} \mathrm{~g} \mathrm{~cm}^{-3}$, $R_{c}=30 \mathrm{AU}$, initial TSC density distribution, $\eta_{\text {star }}=0.3, \theta=15^{\circ}, \mathrm{i}=70^{\circ}$, an inner disk radius of 5 stellar radii, and an outer envelope radius of $10000 \mathrm{AU}$. The $\mathrm{CO}_{2}$ ice abundance was set to $5.0 \times 10^{-5}$. The gray lines represent the model components as in Figure 5 


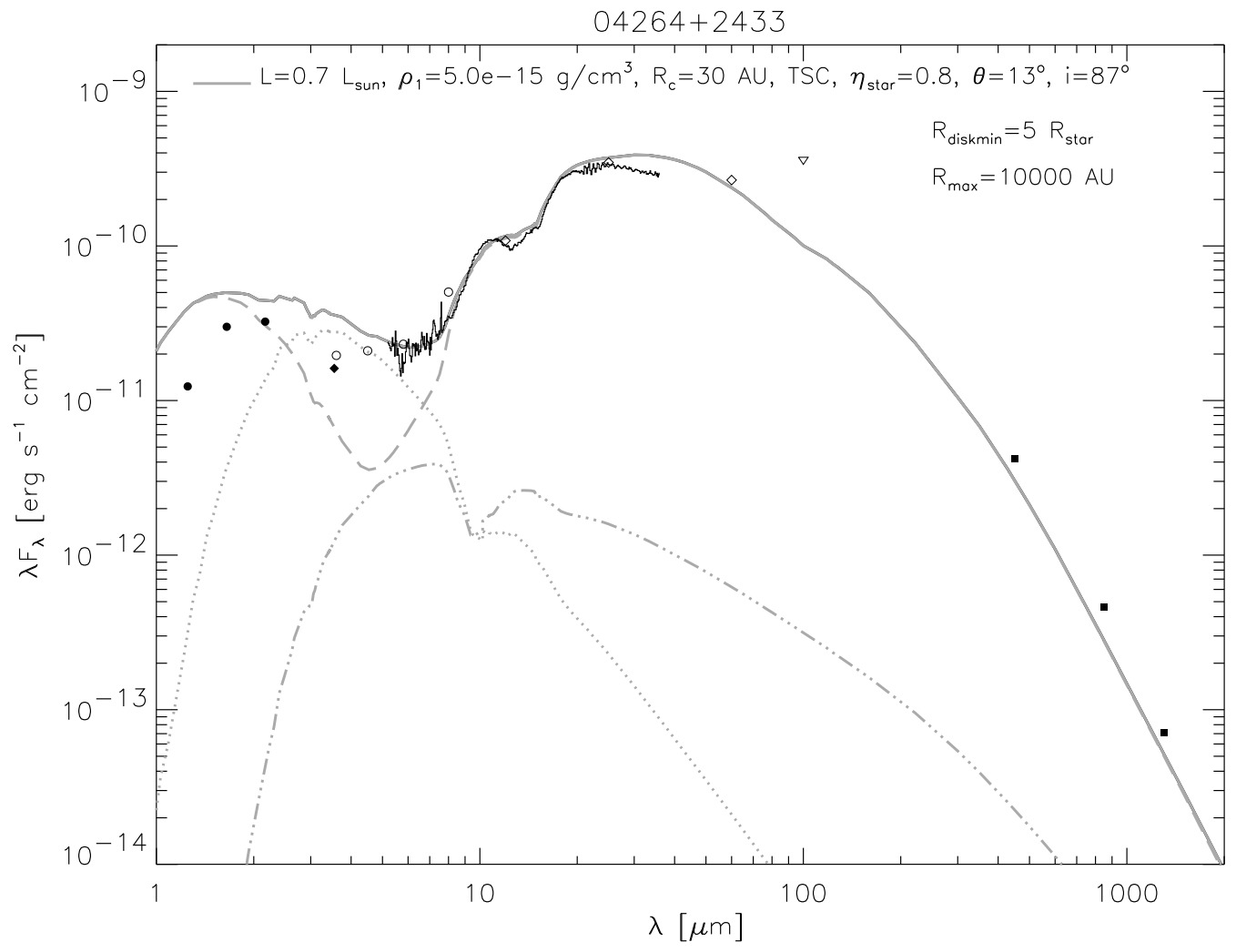

FIG. 15. - IRS spectrum and photometric data of $04264+2433$, and an envelope model fit with $\mathrm{L}=0.7 \mathrm{~L} \odot, \rho_{1}=5.0 \times 10^{-15} \mathrm{~g} \mathrm{~cm}^{-3}$, $R_{c}=30 \mathrm{AU}$, initial TSC density distribution, $\eta_{\text {star }}=0.8, \theta=13^{\circ}, \mathrm{i}=87^{\circ}$, an inner disk radius of 5 stellar radii, and an outer envelope radius of $10000 \mathrm{AU}$. The $\mathrm{CO}_{2}$ ice abundance was set to $5.0 \times 10^{-5}$. The gray lines represent the model components as in Figure 5

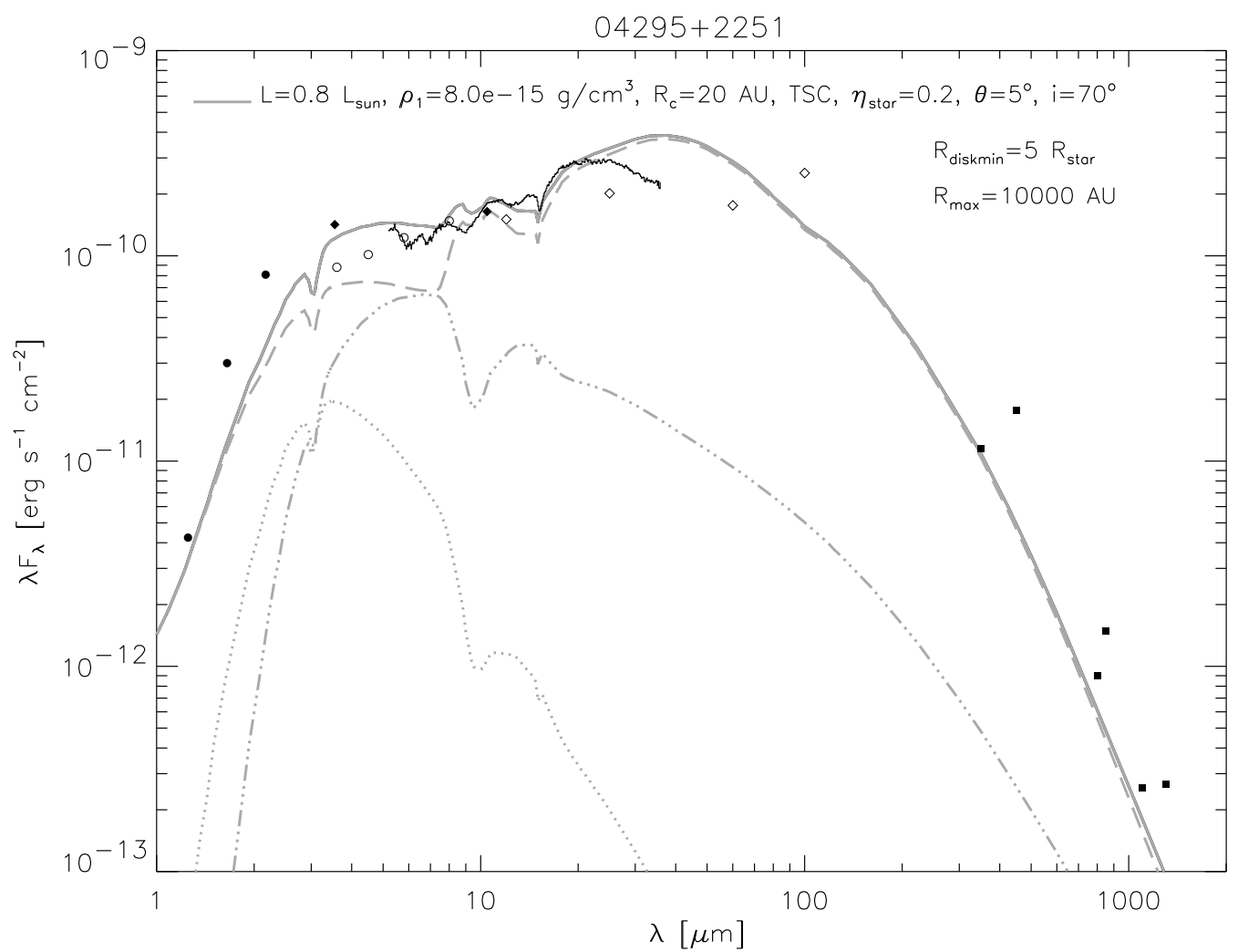

FIG. 16. - IRS spectrum and photometric data of 04295+2251, and an envelope model fit with $\mathrm{L}=0.8 \mathrm{~L} \odot, \rho_{1}=8.0 \times 10^{-15} \mathrm{~g} \mathrm{~cm}^{-3}$, $R_{c}=20 \mathrm{AU}$, initial TSC density distribution, $\eta_{\text {star }}=0.2, \theta=5^{\circ}, \mathrm{i}=70^{\circ}$, an inner disk radius of 5 stellar radii, and an outer envelope radius of $10000 \mathrm{AU}$. The $\mathrm{CO}_{2}$ ice abundance was set to $1.0 \times 10^{-4}$. The gray lines represent the model components as in Figure 5 


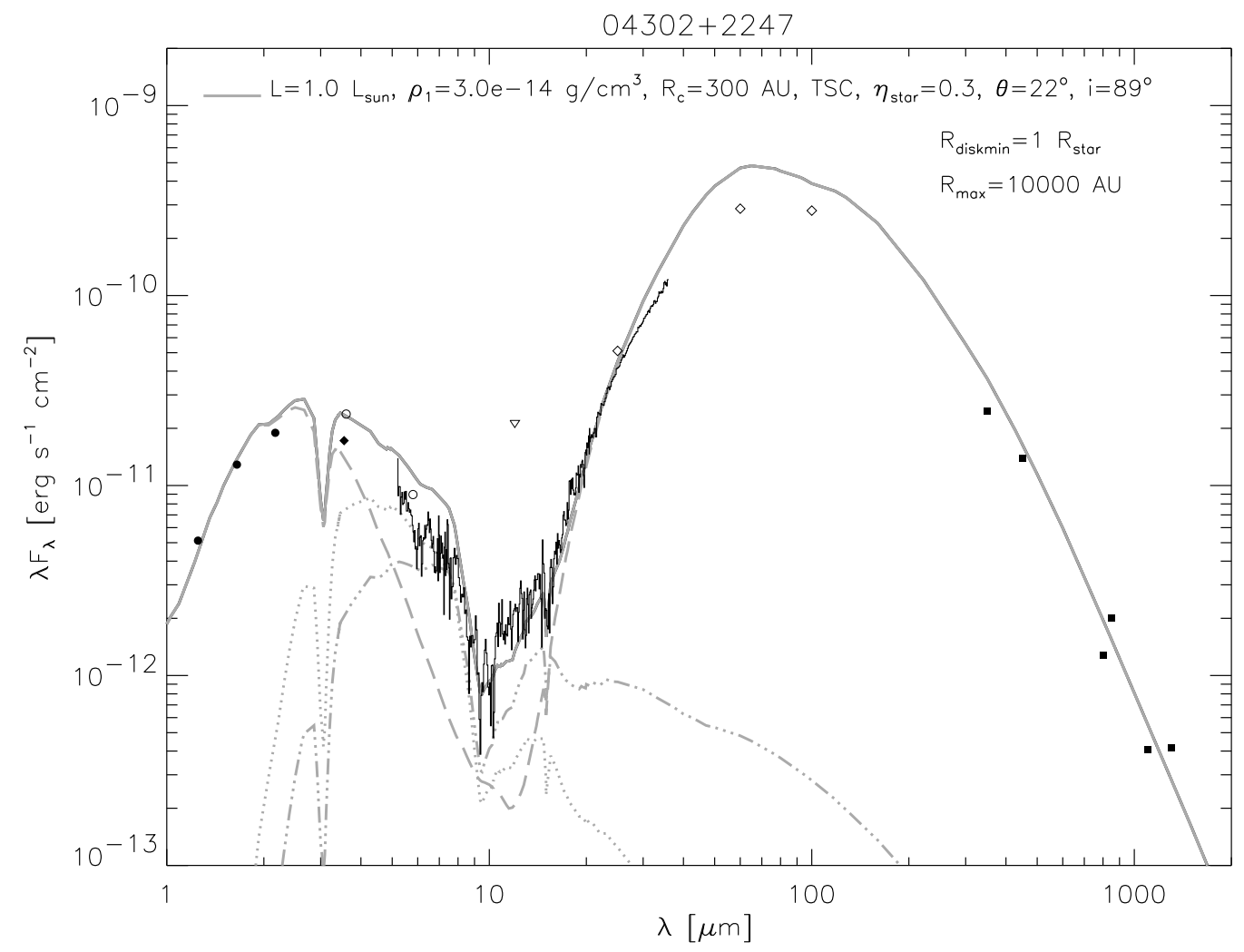

FIG. 17. - IRS spectrum and photometric data of $04302+2247$, and an envelope model fit with $\mathrm{L}=1.0 \mathrm{~L} \odot, \rho_{1}=3.0 \times 10^{-14} \mathrm{~g} \mathrm{~cm}^{-3}$, $R_{c}=300 \mathrm{AU}$, initial TSC density distribution, $\eta_{\text {star }}=0.3, \theta=22^{\circ}, \mathrm{i}=89^{\circ}$, an inner disk radius of 1 stellar radius, and an outer envelope radius of $10000 \mathrm{AU}$. The $\mathrm{CO}_{2}$ ice abundance was set to $4.0 \times 10^{-5}$. The gray lines represent the model components as in Figure 5

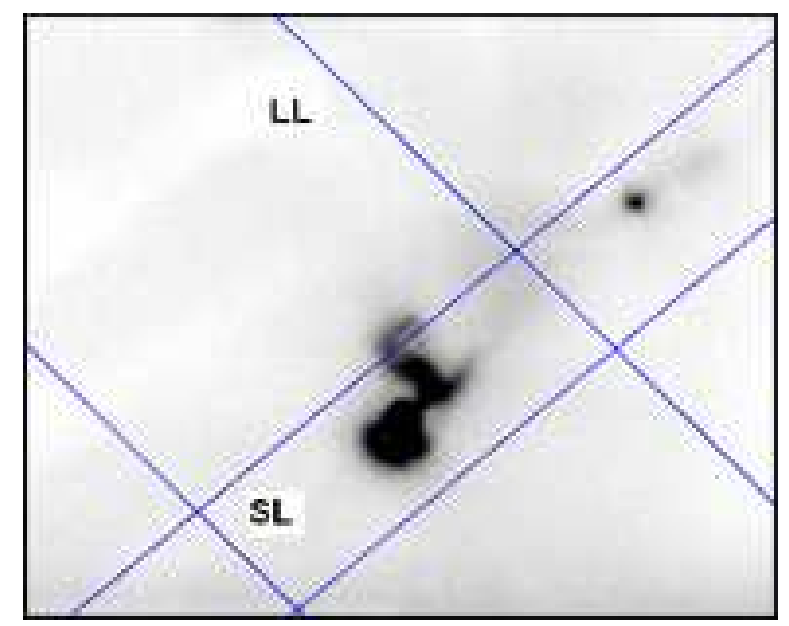

FIG. 18. - The IRS SL and LL slit positions superposed on the NICMOS K-band (F205W) image of IRAS $04325+2402$ by Hartmann et al. (1999). The SL slit (3"'6 wide) runs from the lower left to upper right, while the wider LL slit extends from the upper left to the lower right. The full slit lengths are larger than the size of the image. 


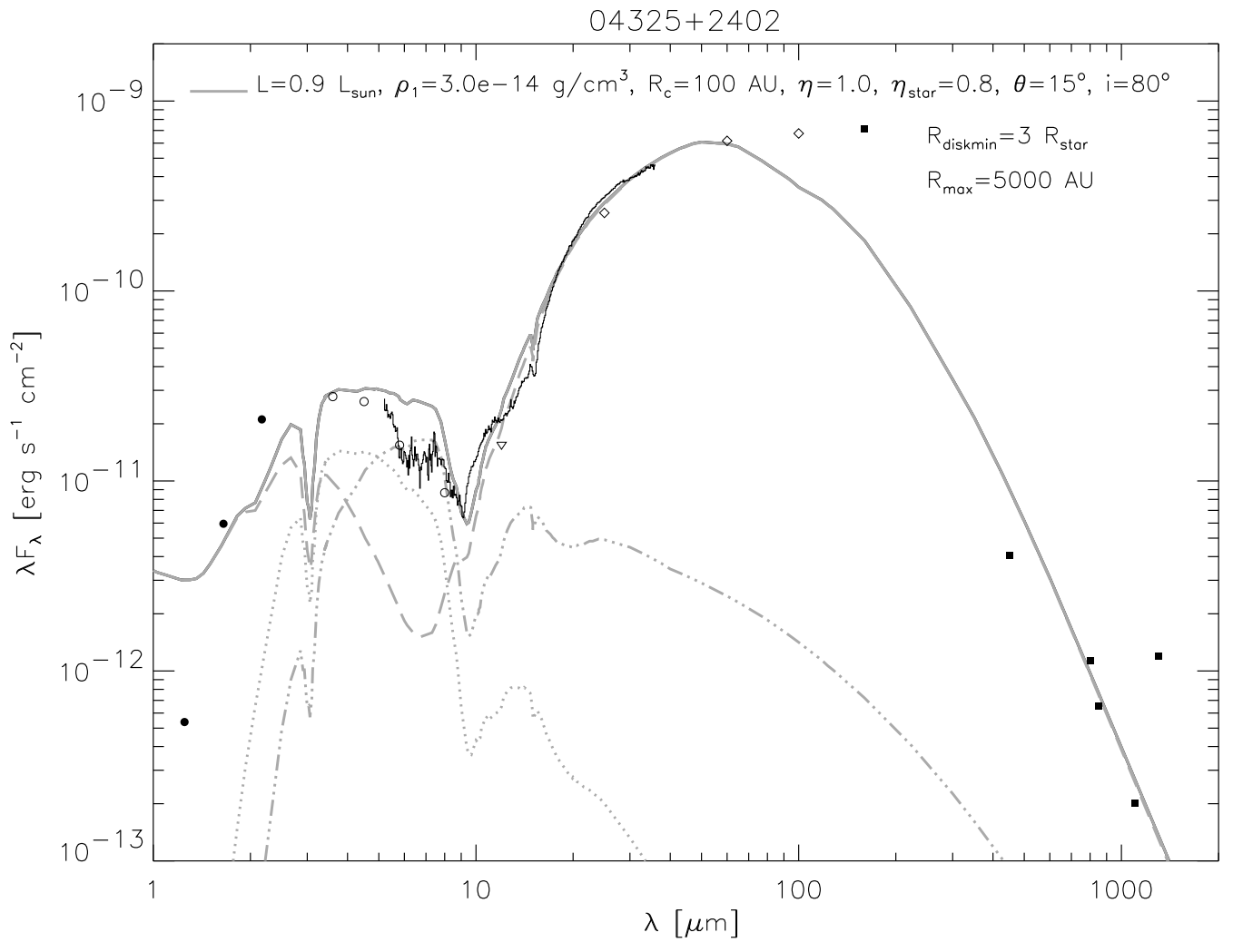

FIG. 19. - IRS spectrum and photometric data of $04325+2402$, and an envelope model fit with $\mathrm{L}=0.9 \mathrm{~L}_{\odot}, \rho_{1}=3.0 \times 10^{-14} \mathrm{~g} \mathrm{~cm}{ }^{-3}$, $R_{c}=100 \mathrm{AU}, \eta=1.0, \eta_{\text {star }}=0.8, \theta=15^{\circ}, \mathrm{i}=80^{\circ}$, an inner disk radius of 3 stellar radii, and an outer envelope radius of $5000 \mathrm{AU}$. The $\mathrm{CO}{ }_{2}$ ice abundance was set to $4.0 \times 10^{-5}$. The gray lines represent the model components as in Figure 5

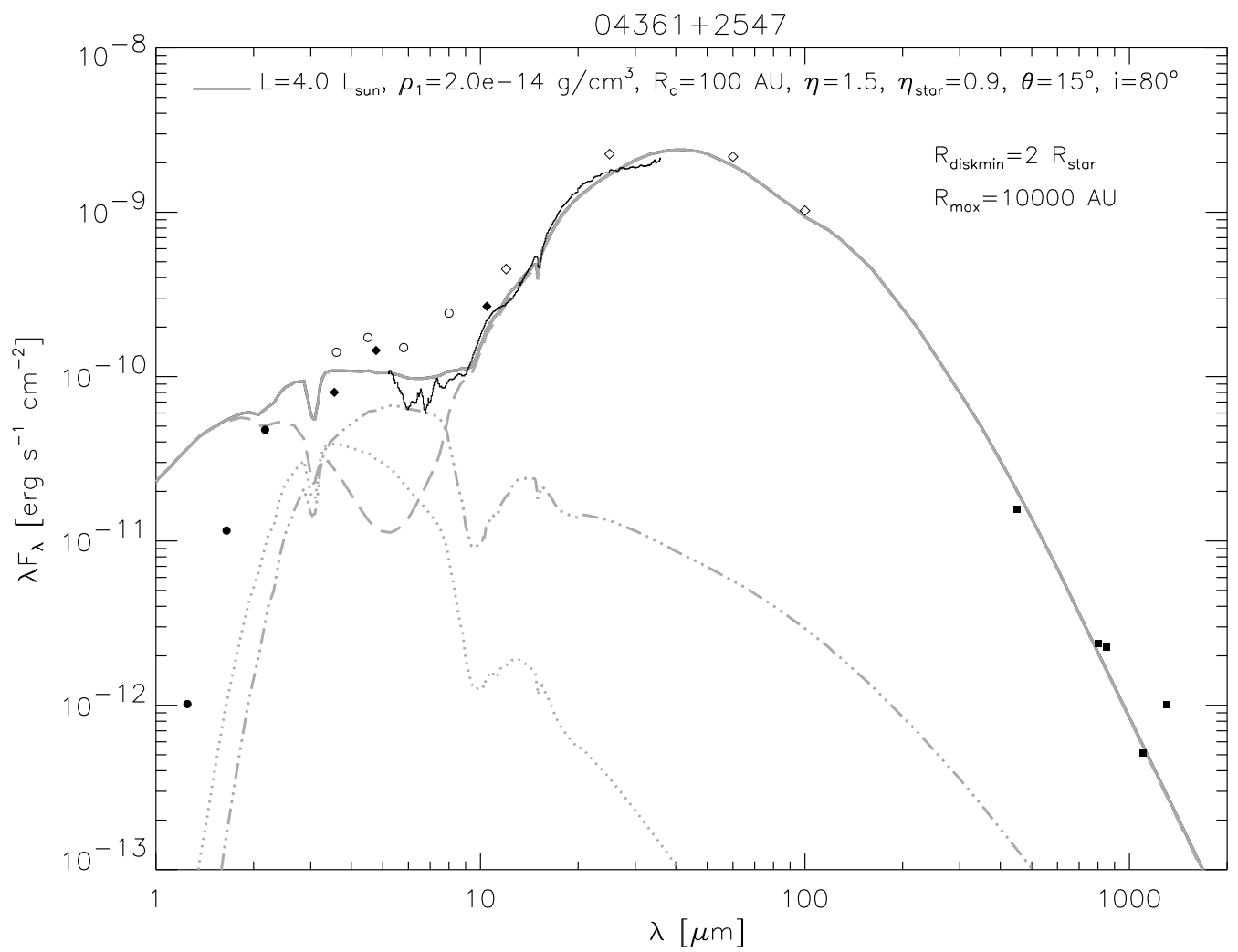

FIG. 20. - IRS spectrum and photometric data of $04361+2547$, and an envelope model fit with $\mathrm{L}=4.0 \mathrm{~L} \odot, \rho_{1}=2.0 \times 10^{-14} \mathrm{~g} \mathrm{~cm}-3$, $R_{c}=100 \mathrm{AU}, \eta=1.5, \eta_{\text {star }}=0.9, \theta=15^{\circ}, \mathrm{i}=80^{\circ}$, an inner disk radius of 2 stellar radii, and an outer envelope radius of $10000 \mathrm{AU}^{\circ}$ The $\mathrm{CO}_{2}$ ice abundance was set to $7.0 \times 10^{-5}$. The gray lines represent the model components as in Figure 5 


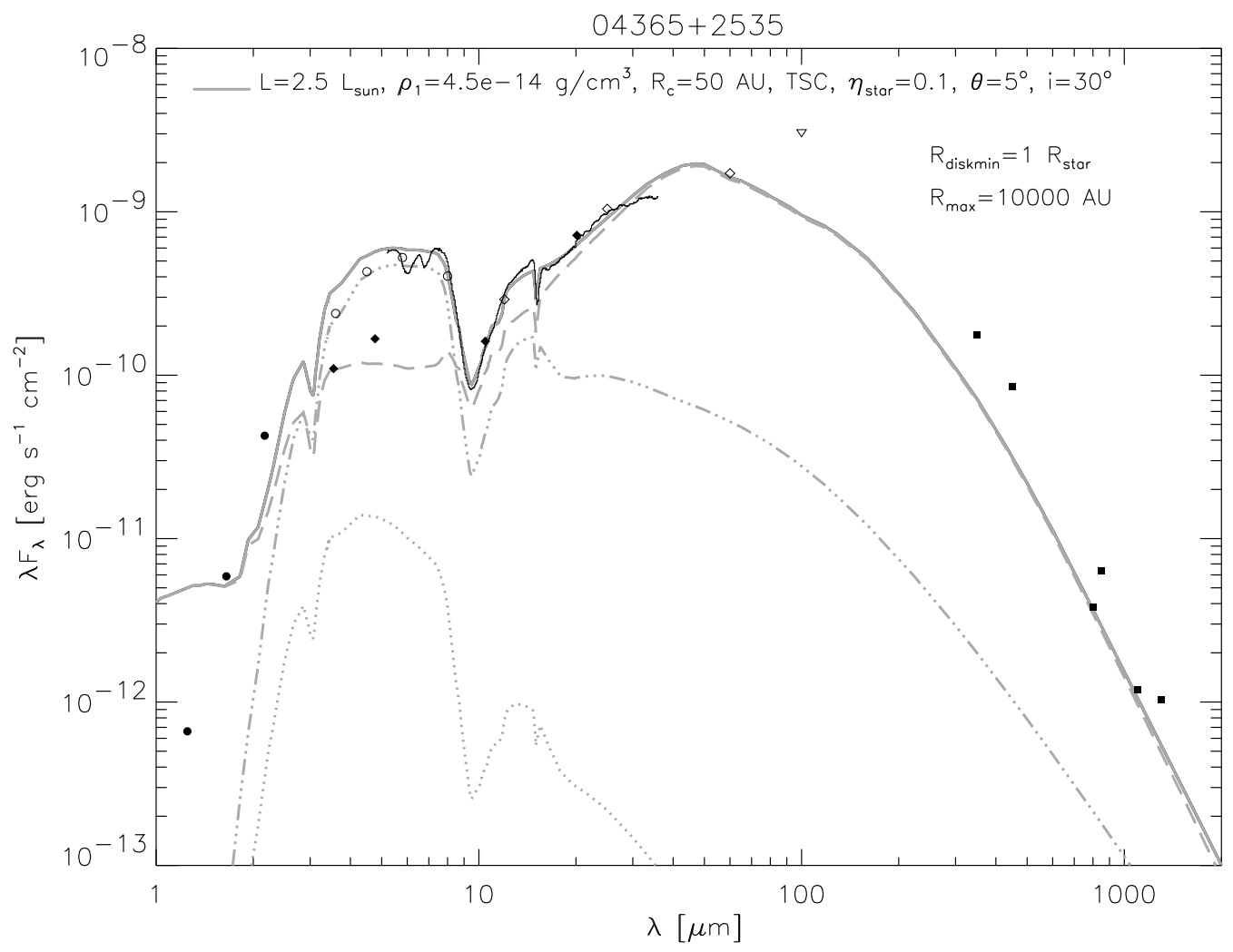

FIG. 21.- IRS spectrum and photometric data of $04365+2535$, and an envelope model fit with $\mathrm{L}=2.5 \mathrm{~L}_{\odot}, \rho_{1}=4.5 \times 10^{-14} \mathrm{~g} \mathrm{~cm}^{-3}$, $R_{c}=50 \mathrm{AU}$, initial TSC density distribution, $\eta_{\text {star }}=0.1, \theta=5^{\circ}, \mathrm{i}=30^{\circ}$, an inner disk radius of 1 stellar radius, and an outer envelope radius of $10000 \mathrm{AU}$. The $\mathrm{CO}_{2}$ ice abundance was set to $7.0 \times 10^{-5}$. The gray lines represent the model components as in Figure 5

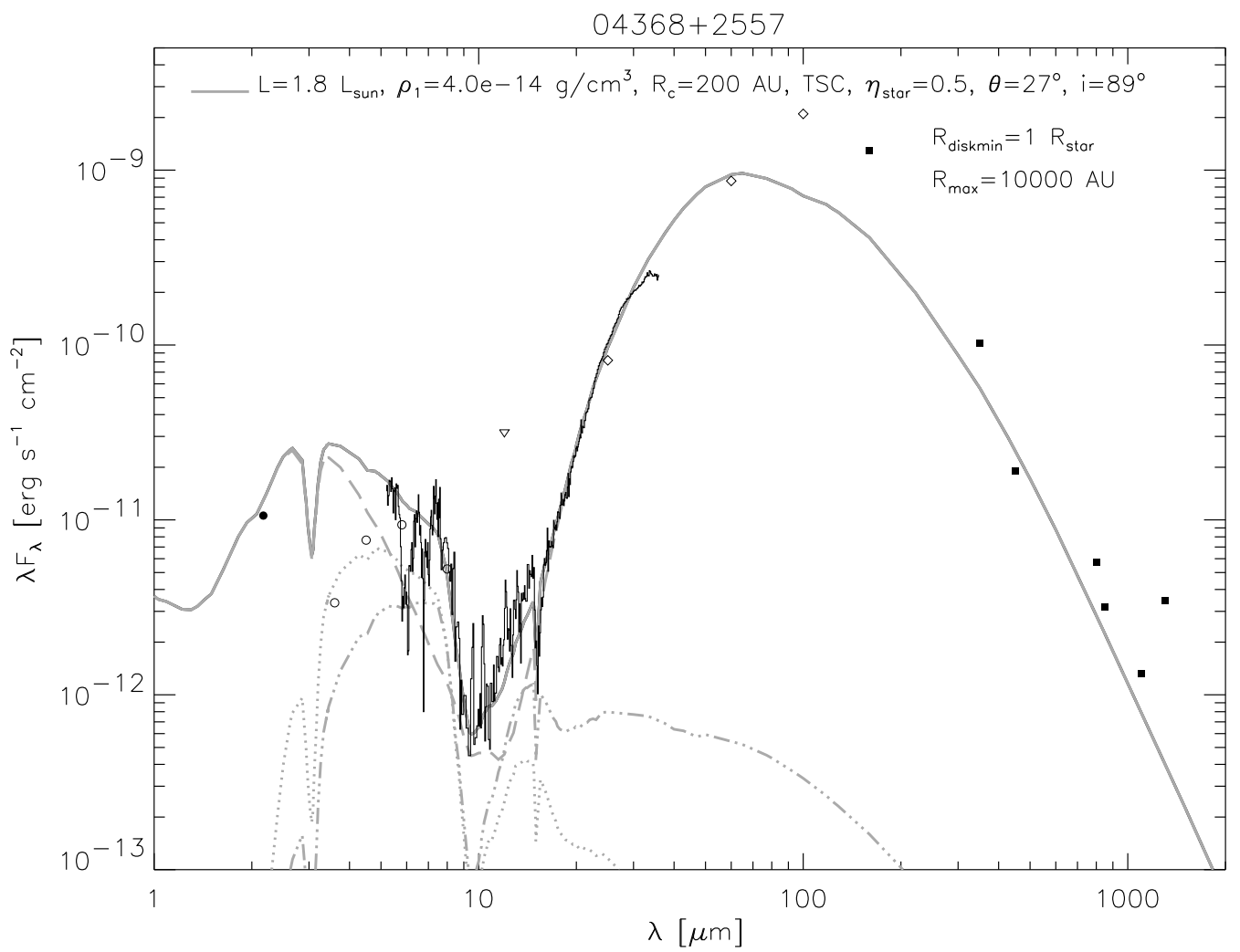

FIG. 22. - IRS spectrum and photometric data of 04368+2557, and an envelope model fit with $\mathrm{L}=1.8 \mathrm{~L} \odot, \rho_{1}=4.0 \times 10^{-14} \mathrm{~g} \mathrm{~cm}^{-3}$, $R_{c}=200 \mathrm{AU}$, initial TSC density distribution, $\eta_{\text {star }}=0.5, \theta=27^{\circ}, \mathrm{i}=89^{\circ}$, an inner disk radius of 1 stellar radius, and an outer envelope radius of $10000 \mathrm{AU}$. The $\mathrm{CO}_{2}$ ice abundance was set to $1.0 \times 10^{-4}$. The gray lines represent the model components as in Figure 5 


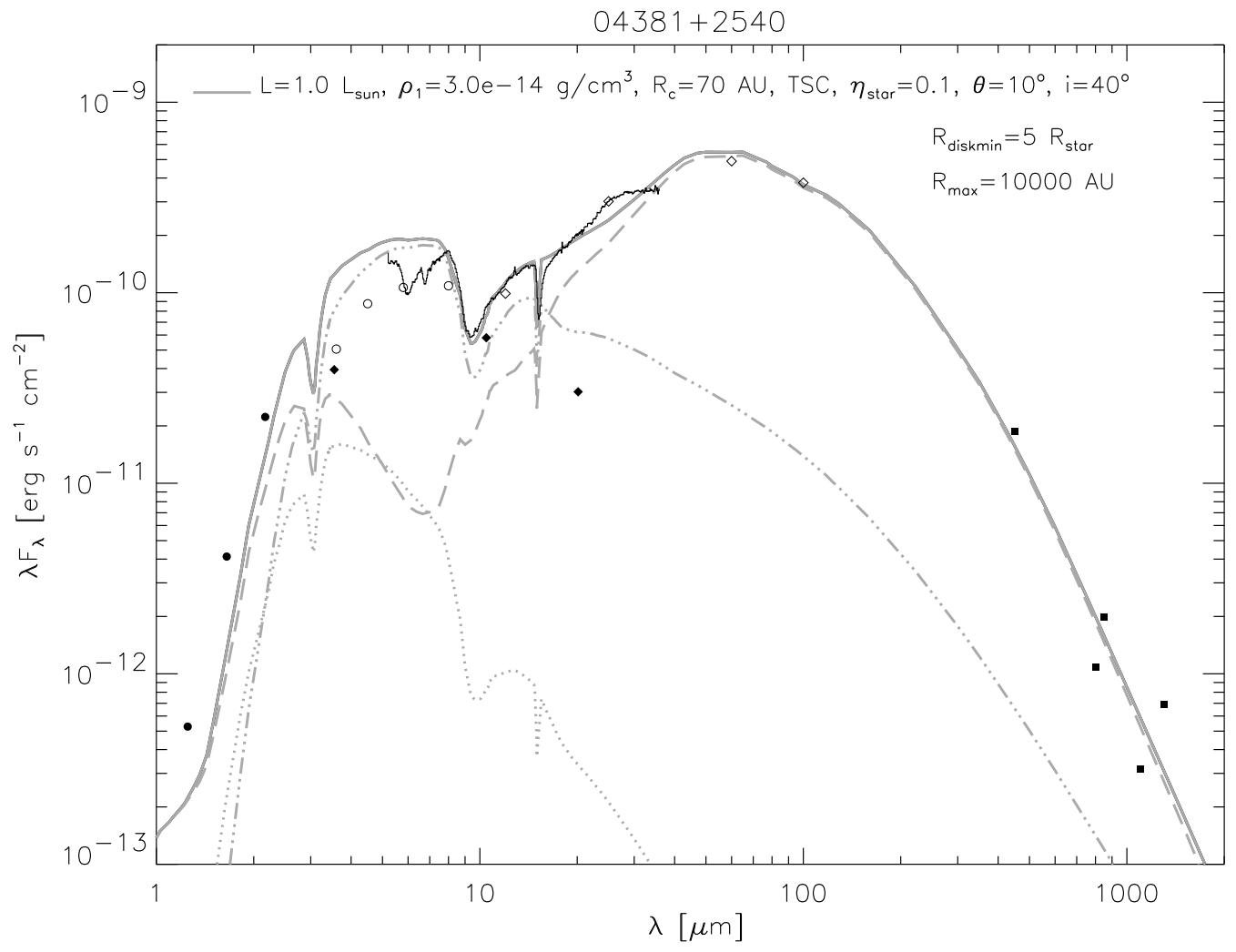

FIG. 23. - IRS spectrum and photometric data of $04381+2540$, and an envelope model fit with $\mathrm{L}=1.0 \mathrm{~L} \odot, \rho_{1}=3.0 \times 10^{-14} \mathrm{~g} \mathrm{~cm}{ }^{-3}$, $R_{c}=70 \mathrm{AU}$, initial TSC density distribution, $\eta_{\text {star }}=0.1, \theta=10^{\circ}, \mathrm{i}=40^{\circ}$, an inner disk radius of 5 stellar radii, and an outer envelope radius of $10000 \mathrm{AU}$. The $\mathrm{CO}_{2}$ ice abundance was set to $1.2 \times 10^{-4}$. The gray lines represent the model components as in Figure 5

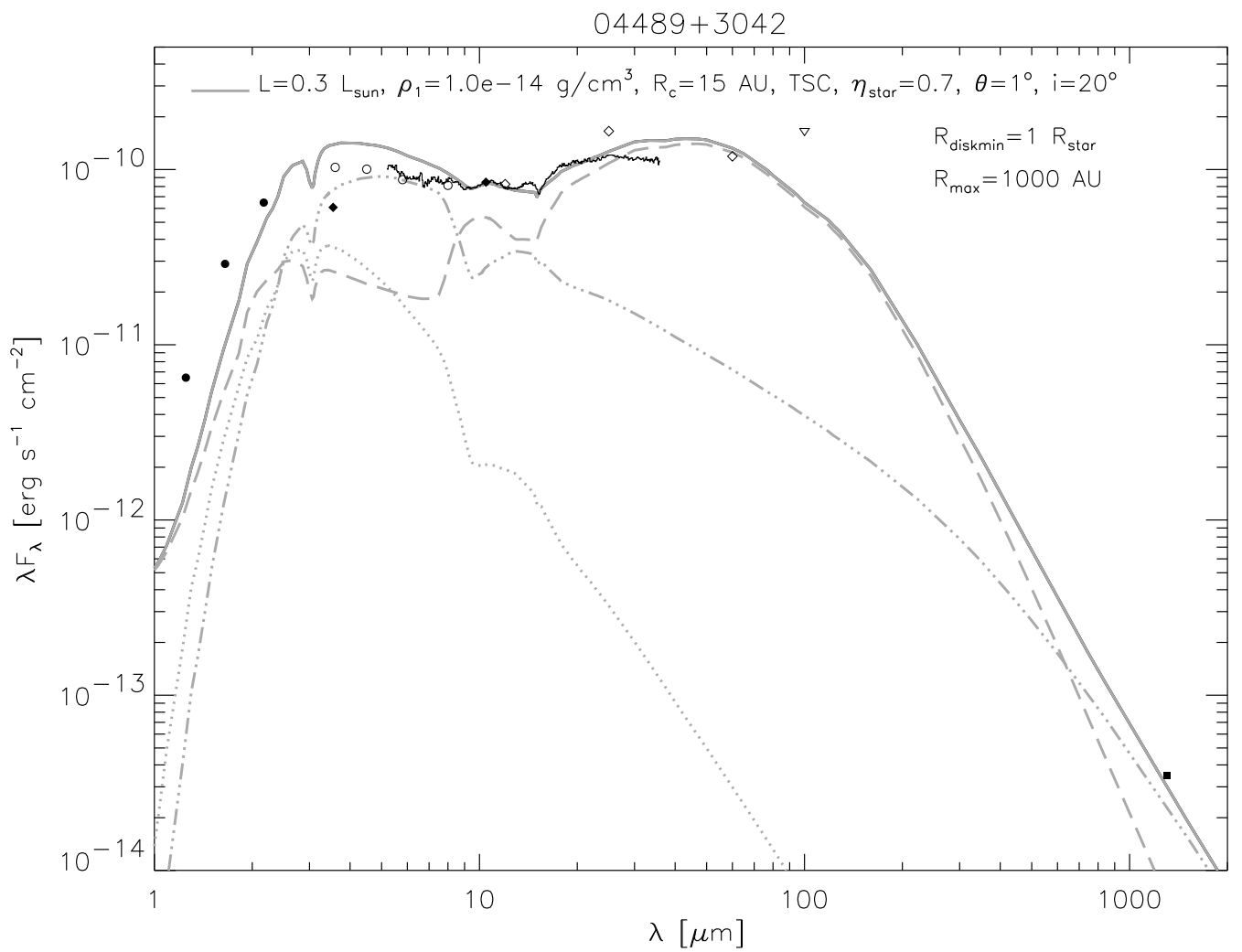

FIG. 24. - IRS spectrum and photometric data of 04489+3042, and an envelope model fit with $\mathrm{L}=0.3 \mathrm{~L} \odot, \rho_{1}=1.0 \times 10^{-14} \mathrm{~g} \mathrm{~cm}^{-3}$, $R_{c}=15 \mathrm{AU}$, initial TSC density distribution, $\eta_{\text {star }}=0.7, \theta=1^{\circ}, \mathrm{i}=20^{\circ}$, an inner disk radius of 1 stellar radius, and an outer envelope radius of $1000 \mathrm{AU}$. The $\mathrm{CO}_{2}$ ice abundance was set to $4.0 \times 10^{-5}$. The gray lines represent the model components as in Figure 5 


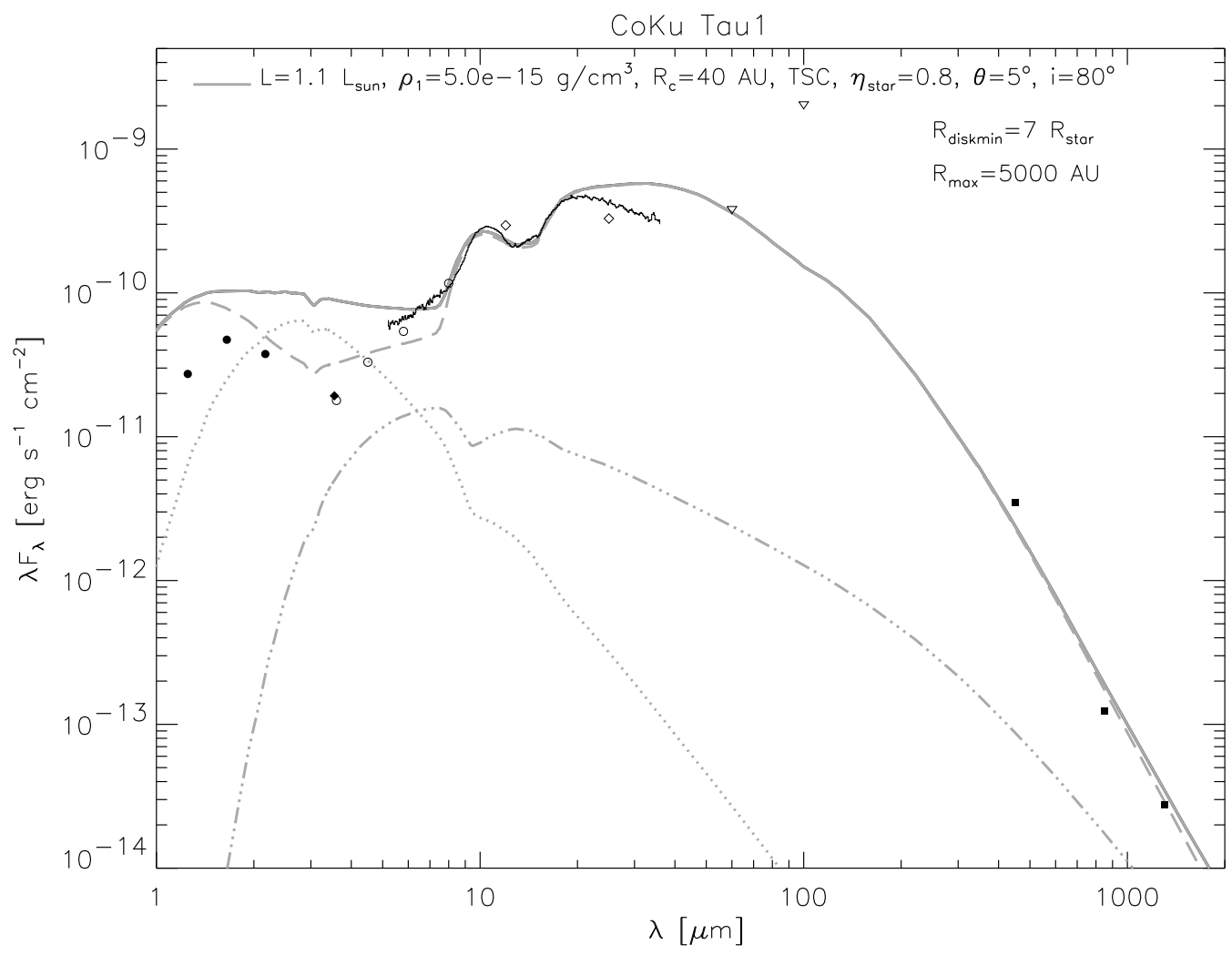

FIG. 25. - IRS spectrum and photometric data of CoKu Tau/1, and an envelope model fit with $\mathrm{L}=1.1 \mathrm{~L}_{\odot}, \rho_{1}=5.0 \times 10^{-15} \mathrm{~g} \mathrm{~cm}^{-3}$, $R_{c}=40 \mathrm{AU}$, initial TSC density distribution, $\eta_{\text {star }}=0.8, \theta=5^{\circ}, \mathrm{i}=80^{\circ}$, an inner disk radius of 7 stellar radii, and an outer envelope radius of $5000 \mathrm{AU}$. The $\mathrm{CO}_{2}$ ice abundance was set to $5.0 \times 10^{-5}$. The gray lines represent the model components as in Figure 5

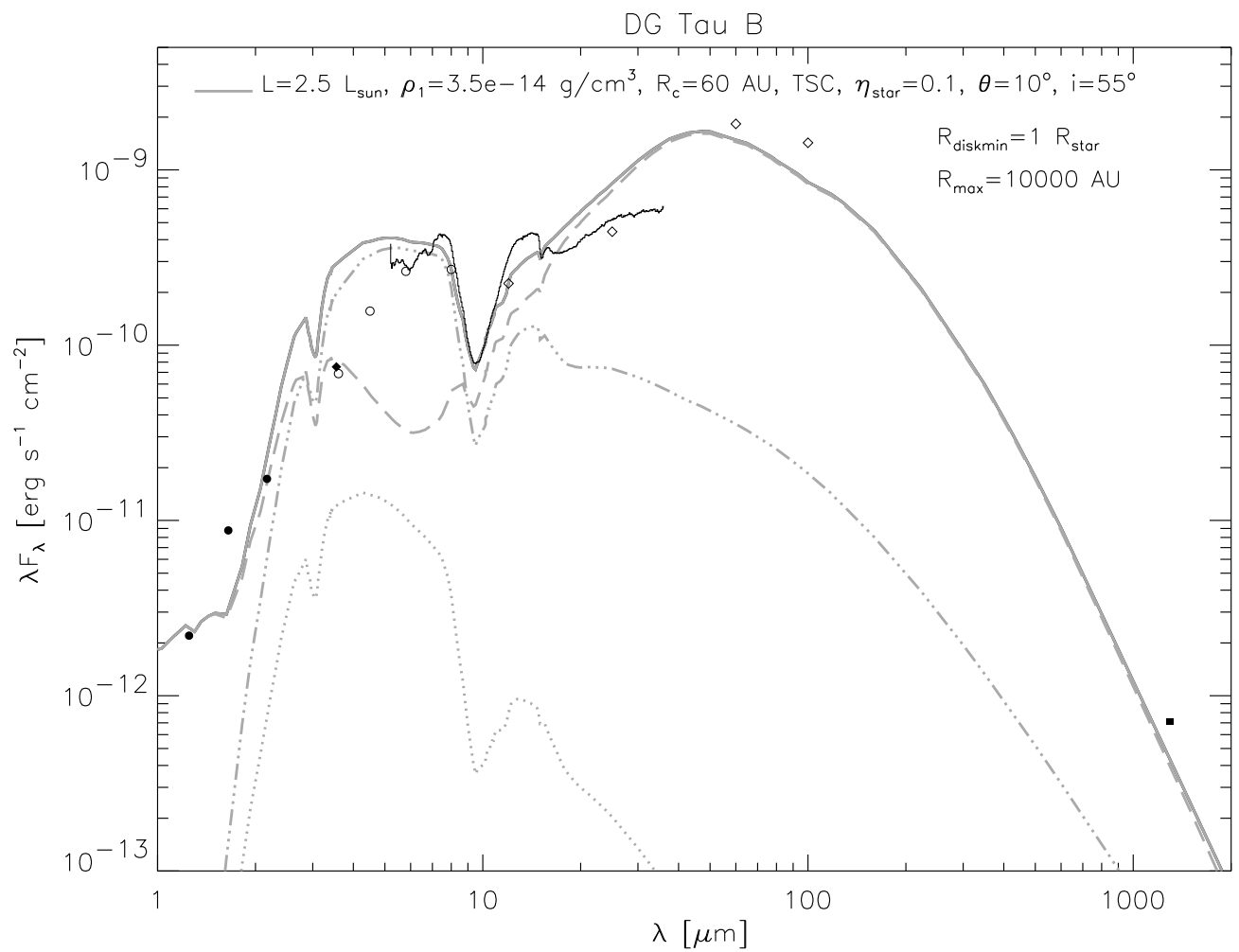

FIG. 26. - IRS spectrum and photometric data of DG Tau B, and an envelope model fit with $\mathrm{L}=2.5 \mathrm{~L} \odot, \rho_{1}=3.5 \times 10^{-14} \mathrm{~g} \mathrm{~cm}^{-3}$, $R_{c}=60 \mathrm{AU}$, initial TSC density distribution, $\eta_{\text {star }}=0.1, \theta=10^{\circ}$, i $=55^{\circ}$, an inner disk radius of 1 stellar radius, and an outer envelope radius of $10000 \mathrm{AU}$. The $\mathrm{CO}_{2}$ ice abundance was set to $3.0 \times 10^{-5}$. The gray lines represent the model components as in Figure 5 


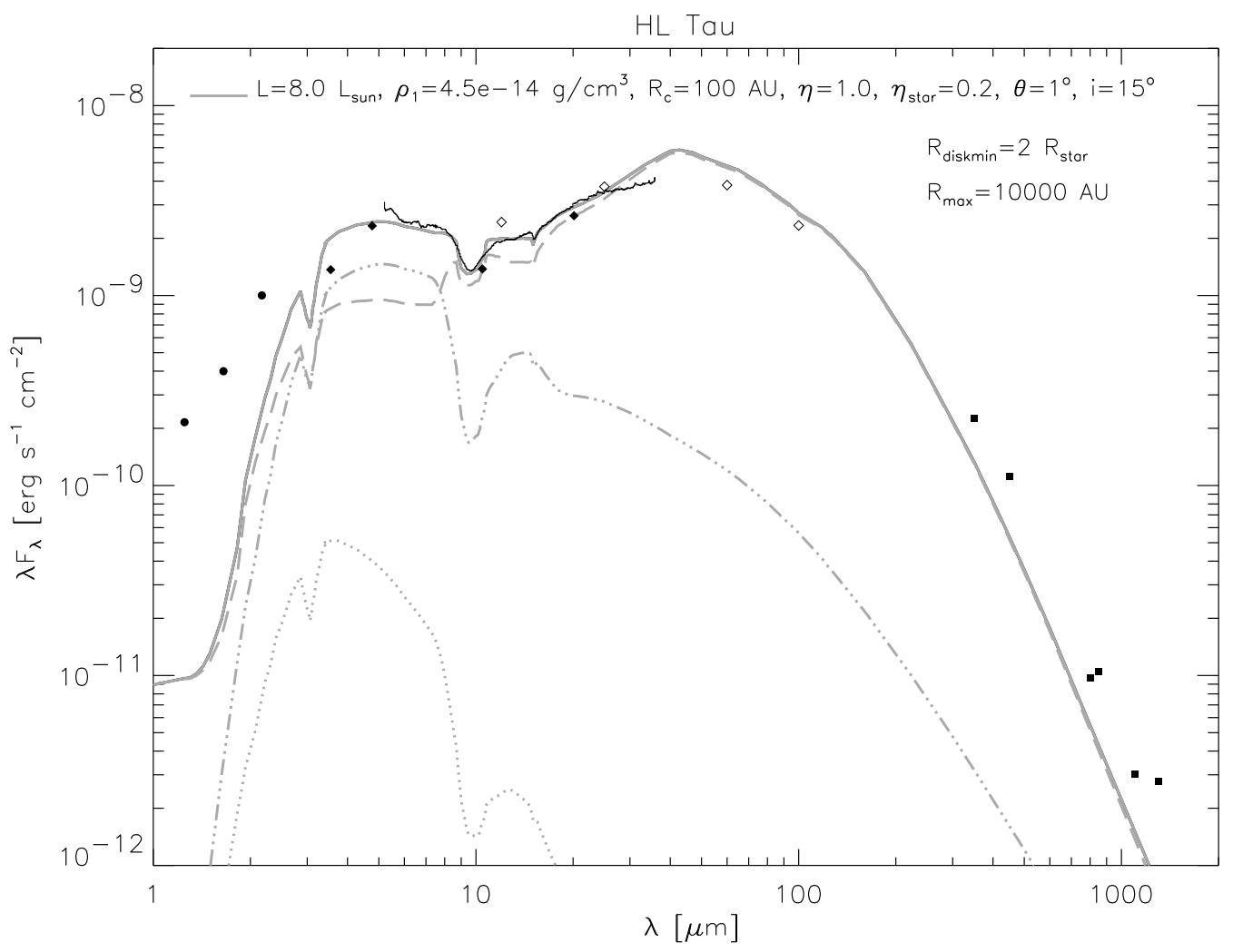

FIG. 27.- IRS spectrum and photometric data of HL Tau, and an envelope model fit with $\mathrm{L}=8.0 \mathrm{~L} \odot, \rho_{1}=4.5 \times 10^{-14} \mathrm{~g} \mathrm{~cm}-3, R_{c}=100$ AU, $\eta=1.0, \eta_{\text {star }}=0.2, \theta=1^{\circ}, \mathrm{i}=15^{\circ}$, an inner disk radius of 2 stellar radii, and an outer envelope radius of $10000 \mathrm{AU}^{\mathrm{T}} \mathrm{The} \mathrm{CO}_{2}$ ice abundance was set to $4.0 \times 10^{-5}$. The gray lines represent the model components as in Figure 5

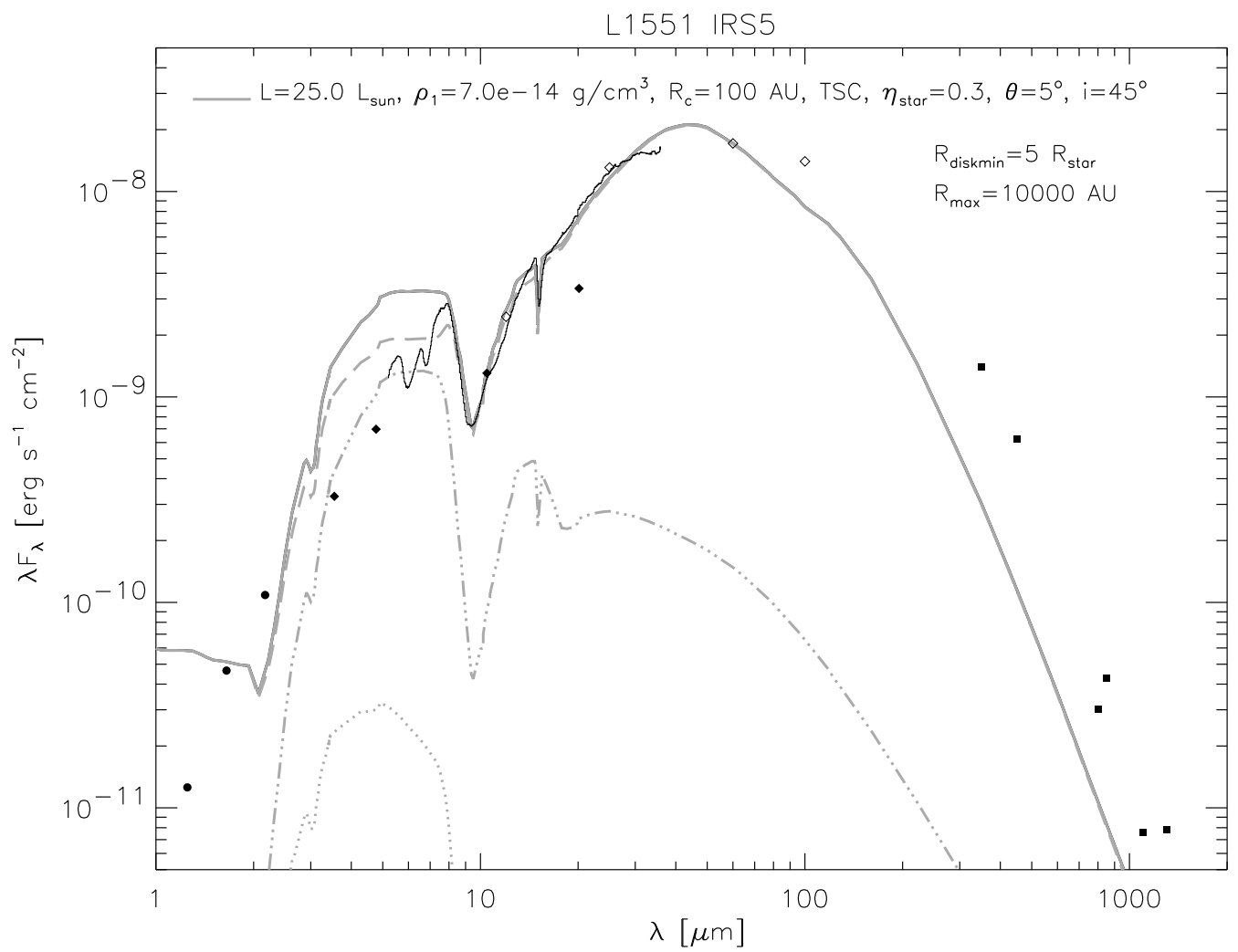

FIG. 28. - IRS spectrum and photometric data of L1551 IRS 5, and an envelope model fit with $\mathrm{L}=25.0 \mathrm{~L}_{\odot}, \rho_{1}=7.0 \times 10^{-14} \mathrm{~g} \mathrm{~cm}-3$, $R_{c}=100 \mathrm{AU}$, initial TSC density distribution, $\eta_{\text {star }}=0.3, \theta=5^{\circ}, \mathrm{i}=45^{\circ}$, an inner disk radius of 5 stellar radii, and an outer envelope radius of $10000 \mathrm{AU}$. The $\mathrm{CO}_{2}$ ice abundance was set to $3.0 \times 10^{-4}$. The gray lines represent the model components as in Figure 5 
Spitzer Spectra and Models of Protostars in Taurus

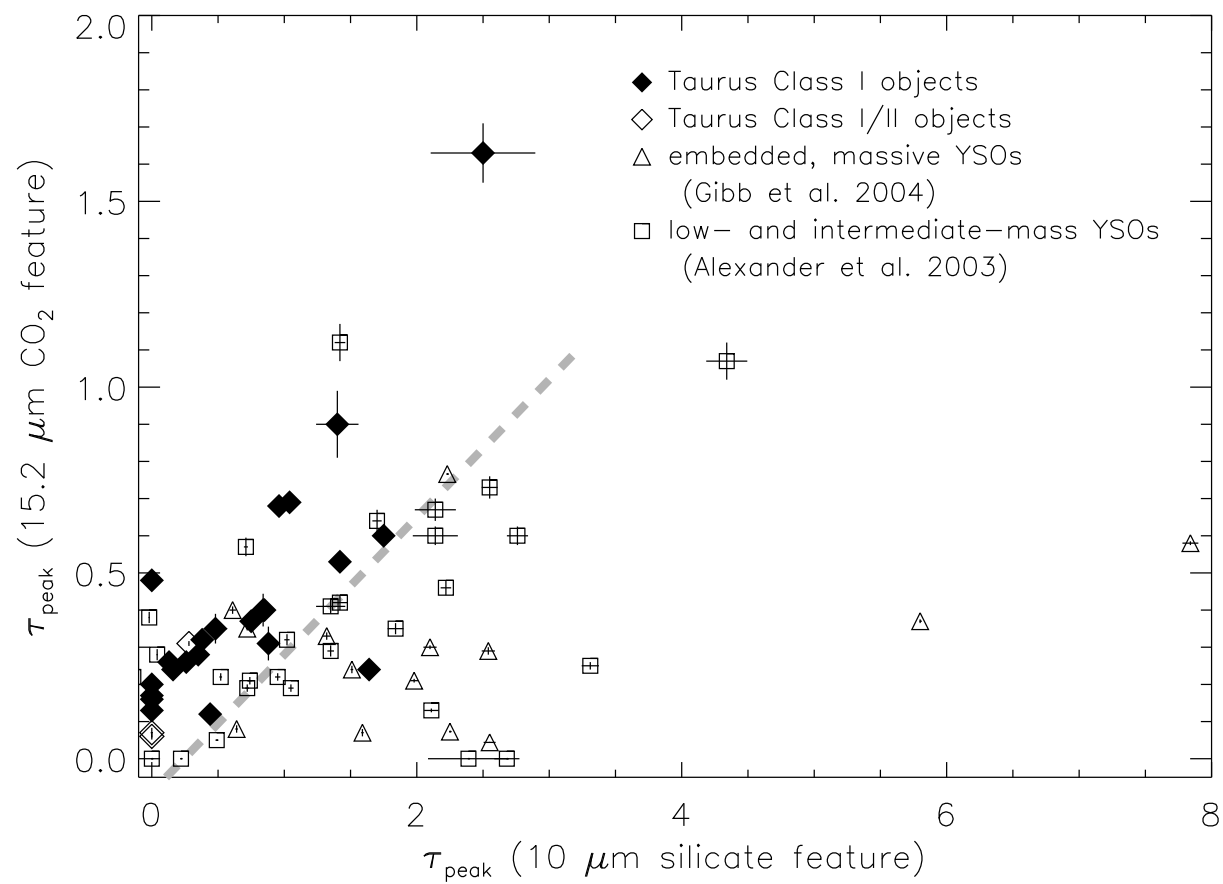

FIG. 29.- Peak optical depth of the $\mathrm{CO}_{2}$ ice feature versus the peak optical depth of the $10 \mu \mathrm{m}$ silicate absorption feature. The diamonds represent the Class I objects presented in this paper, while the triangles and squares represent generally more massive YSOs studied by Gibb et al. (2004) and Alexander et al. (2003), respectively. The data points at a peak silicate optical depth of 0 are objects with either a silicate emission feature or a feature that shows emission and absorption characteristics. The thick, gray dashed line is drawn to guide the eye to separate the regimes of envelopes from that of ambient molecular cloud material (see text for details). 